TITLE:

\title{
Crystal-plasticity finite-element analysis of anisotropic deformation behavior in a commercially pure titanium Grade 1 sheet
}

\section{$\operatorname{AUTHOR}(S):$}

Hama, Takayuki; Kobuki, Akihiro; Takuda, Hirohiko

\section{CITATION:}

Hama, Takayuki ...[et al]. Crystal-plasticity finite-element analysis of anisotropic deformation behavior in a commercially pure titanium Grade 1 sheet. International Journal of Plasticity 2017, 91: 77-108

\section{ISSUE DATE:}

2017-04

URL:

http://hdl.handle.net/2433/228176

\section{RIGHT:}

(c) 2017. This manuscript version is made available under the CC-BY-NC-ND 4.0 license

http://creativecommons.org/licenses/by-nc-nd/4.0/; The full-text file will be made open to the public on 01 April 2019 in accordance with publisher's 'Terms and Conditions for Self-Archiving'; この論文は出版社版でありません。引用の際に は出版社版をご確認ご利用ください。; This is not the published version. Please cite only the published version. 
Crystal-plasticity finite-element analysis of anisotropic deformation behavior in a commercially pure titanium Grade 1 sheet

Takayuki Hama*

Akihiro Kobuki

Hirohiko Takuda

Graduate School of Energy Science, Kyoto University, Yoshida-honmachi, Sakyo-ku, Kyoto 606-8501, Japan

Corresponding author:

Takayuki Hama

Contact address: Graduate School of Energy Science, Kyoto University, Yoshida-honmachi, Sakyo-ku, Kyoto 606-8501, Japan

Tel.: +81 (0)75-753-5418; Fax: +81 (0)75-753-5428

E-mail address: hama@energy.kyoto-u.ac.jp

Highlights

- Deformation behavior in a CP-Ti sheet was studied using crystal-plasticity FEM.

- The material parameters were determined based on the role of each deformation mode.

- The deformation under various strain paths was predicted well using the simulation.

- The deformation mechanism was examined numerically from a mesoscopic viewpoint. 


\section{Abstract}

A crystal-plasticity finite-element method was used to study the deformation behavior of a commercially pure titanium Grade 1 sheet upon different strain paths. Prismatic slip, pyramidal $\langle a\rangle$ slip, basal slip, two types of pyramidal $\langle a+c\rangle$ slip, $\{10 \overline{1} 2\}$ twinning, and $\{11 \overline{2} 2\}$ twinning were taken into consideration. The material parameters were systematically determined considering the role of each active deformation mode. The simulation results were in good agreement with the experimental results with respect to evolution of the Lankford value, stress-strain curves, contours of plastic work, and texture evolution for the strain paths examined in this study. The mechanism of anisotropic deformation behavior was then investigated, focusing especially on the role of the activity of twinning in the plastic deformation. It was found that the twinning activity significantly affected the following characteristics: the anisotropies in the Lankford value and work hardening under compression and the tension-compression asymmetries in the stress-strain curves in the rolling direction. The detwinning activity also affected stress-strain curves upon reverse loading, in particular in the rolling direction. To systematically understand the deformation mechanism, the effect of slip activity on the deformation behavior is also discussed.

\section{Keywords:}

B. Crystal plasticity; C. Finite elements; B. Anisotropic material; A. Twinning; Reverse loading 


\section{Introduction}

Owing to its high strength, corrosion resistance, burning resistance, and biological compatibility, commercially pure (CP) $\alpha$-titanium (Ti) sheets have widely been used in chemical plants, consumer products, and heat exchangers [Ishiki et al., 2009, 2011; Zeng et al., 2009], and they are expected to be further used in new applications [Hayashi, 2001].

CP-Ti sheets show strong anisotropic deformation, which is much more pronounced than that of other sheet metals that can be used for structural components such as steel and aluminum alloy sheets. Because the anisotropic property significantly affects the press formability, a number of studies have been done to understand the macroscopic deformation behavior in CP-Ti sheets [Ishiki et al., 2009, 2011; Huang et al., 2010; Lee and Backofen, 1966; Mullins and Patchett, 1981; Nixon et al., 2010; Bouvier et al., 2012; Ishiyama, 2006; Nasiri-Abarbekoh et al., 2012]. Ishiki et al. (2009, 2011) carried out uniaxial tension, uniaxial compression, and biaxial tension and compression tests for a CP-Ti sheet. They showed that the CP-Ti sheet presented strong in-plane anisotropy in the work-hardening behavior and evolution of the Lankford value (the $r$ value) under monotonic tension. In addition, stress-strain curves obtained in the rolling direction (RD) exhibited pronounced tension-compression asymmetry. Bouvier et al. (2012) performed a simple shear test in a CP-Ti sheet along various directions under monotonic, reverse, and cyclic loadings. They reported that in-plane anisotropy was observed under simple shear as well.

The anisotropy shown in CP-Ti sheets is attributed to the hexagonal close-packed (hcp) structure that exhibits crystal anisotropy [Mullins and Patchett, 1981; Nixon et al., 2010; Ishiyama, 2006; Conrad, 1981]. Specifically, the primary slip system in CP-Ti sheets is prismatic $<a>$ slip; basal $<a>$ slip, pyramidal $<a>$ slip, and pyramidal $<a+c>$ slip are also active [Conrad, 1981; Nemat-Nasser et al., 1999; Warwick et al., 2012; Chichili et al., 1998; Rossi et al., 1953; Anderson et al., 1953; Wang et al., 2011; Paton and Backofen, 1970; Numakura et al., 1986]. However, the critical resolved shear stress (CRSS), as well as work hardening, differs significantly depending on the family of slip systems. Moreover, in rolled CP-Ti sheets, strong basal texture, with the $c$-axes tilted between 20 and $40^{\circ}$ from the normal direction (ND) to the transverse direction (TD), is exhibited; therefore, the anisotropic deformation is further pronounced. For instance, when the sheet is subjected to plastic deformation along the $\mathrm{RD}$, the activity of $\langle a\rangle$ slip systems hardly contributes to the deformation in the thickness direction because of the strong transverse-split basal texture. 
It has also been established that in hcp metals, the activity of twinning systems [Mullins and Patchett, 1981; Ishiyama, 2006; Conrad, 1981; Paton and Backofen, 1970; Chun et al., 2005; Christian and Mahajan, 1995] plays an important role in the deformation behavior. A number of studies have reported a variety of active twinning systems in CP-Ti [Mullins and Patchett, 1981; Conrad, 1981; Paton and Backofen, 1970]. Among them, the activities of $\{10 \overline{1} 2\}$ tensile twinning and $\{11 \overline{2} 2\}$ compressive twinning systems are large at room temperature [Hama et al., 2014]. Moreover, active twinning systems are different depending on the loading condition because of their direction dependence in deformation. For instance, in rolled CP-Ti sheets, $\{11 \overline{2} 2\}$ and $\{10 \overline{1} 2\}$ twinning systems are easily activated under in-plane tension and compression, respectively [Hama et al., 2014; Yi et al., 2016].

A number of recent studies have examined the mechanisms of macroscopic anisotropy under monotonic loading with respect to the microscopic deformation behavior [Nixon et al., 2010; Ishiyama, 2006; Nemat-Nasser et al., 1999; Roth et al., 2014; Tirry et al., 2012; Yu et al., 2008; Ishiyama et al., 1990; Battaini et al., 2007]. Nixon et al. (2010) conducted monotonic tension and compression tests and studied the mechanism of tension-compression anisotropy. The amount of twinning activated under compression was much more pronounced as compared to that under tension in the RD at strains larger than $10 \%$. This difference accounted for greater work hardening under compression than under tension at strains larger than 10\%. Ishiyama et al. (1990) reported that in-plane anisotropy presented under tension could be explained in terms of the direction dependence of twinning activity. Battaini et al. (2007) conducted plane strain compression using samples prepared along different orientations. They concluded that the difference in the flow stresses among samples with different orientations largely depended on the activity of prismatic slip and twinning.

It is important to understand the deformation behavior not only under monotonic loading but also under reverse and biaxial loadings. For instance, a sheet is often subjected to reverse or biaxial loadings when it is manufactured by press forming. A few studies investigated deformation characteristics and mechanisms under reverse and biaxial loadings in CP-Ti sheets experimentally [Ishiki et al., 2009, 2011; Hama et al., 2014; Yi et al., 2016]. The present authors [Yi et al., 2016] studied the anisotropic deformation behavior upon monotonic, reverse, and cyclic loadings in CP-Ti Grade 1 and 2 sheets. The twinning and detwinning activities under reverse loading in the Grade 1 sheet were much larger than those in the Grade 2 sheet, yielding more pronounced anisotropic deformation in the Grade 1 sheet. Ishiki et al. (2009, 2011) studied the deformation behavior during biaxial tension and compression under linear stress paths. 
They reported that the anisotropic work-hardening behavior was observed in the first, second, and fourth quadrants of the stress space and that the tendency of anisotropy was different depending on the quadrant. Moreover, they also reported that the change in the shape ratio of the counters of plastic work was larger in the first quadrant than in the other quadrants.

Despite the abovementioned studies, it is quite difficult to further investigate the deformation mechanism in CP-Ti sheets experimentally. This is because various slip and twinning systems are active during deformation, as described earlier; thus, it is difficult to understand the role of each deformation mode on the work-hardening behavior experimentally. Moreover, the active deformation mode would change with the progress of deformation because of family-dependent CRSS and work hardening and the easy evolution of texture owing to the twinning activity. Additionally, although it is understood that the twinning activity yields large work hardening [Bouaziz and Guelton, 2001; Ishiyama, 2006; Bouaziz et al., 2008; Nixon et al., 2010], it is difficult to investigate this characteristic quantitatively and comprehensively only by means of experiment. For instance, the present authors studied the work-hardening and twinning behaviors during monotonic and reverse loadings in CP-Ti sheets experimentally in previous studies [Hama et al., 2014; Yi et al., 2016]. In these studies, the effect of twinning activity on the work-hardening behavior could be discussed only for limited cases, and it was only on the basis of literatures. Moreover, active slip modes and their effect on the work-hardening behavior could not be discussed at all. As a result, the mechanism that yielded the anisotropic work-hardening behavior was not examined in detail. Another example is as follows. The anisotropic deformation behavior under biaxial tension that occurs in CP-Ti is presumably due to the fact that the active deformation mode is different depending on the stress ratio and changes in the plastic deformation process. However, it is almost impossible to verify this presumption experimentally.

The crystal-plasticity model is an effective tool for understanding the mechanism of macroscopic deformation from mesoscopic crystalline deformation in metals. This technique has increasingly been applied to hcp metals, particularly magnesium (Mg) and its alloys [Graff et al., 2007; Hama and Takuda 2011; Hama and Takuda 2012a; Wang et al., 2013; Hama et al., 2013, 2016;], and it is utilized to study their deformation mechanisms. There are a few previous studies concerned with a crystal-plasticity analysis in CP-Ti [Wu et al., 2007; Zambaldi et al., 2012; Yang et al., 2011; Gurao et al., 2011; Benmhenni et al., 2013; Kowalczyk-Gajewska et al., 2015; Sinha et al., 2016; Amouzou et al., 2016; Gloaguen et al., 2016; Marchenko et al., 2016]. Wu et al. (2007) 
examined the texture evolution and anisotropic stress-strain response under monotonic deformation paths. They proposed a new Taylor-type polycrystalline model and predicted deformation behavior under monotonic loading along different directions. Warwick et al. (2012) studied lattice strain evolution during tension and compression of CP-Ti. They utilized neutron diffraction, microscopy, and a self-consistent model to examine the micromechanics of CP-Ti. However, the abovementioned studies focused only on simple loading conditions such as monotonic tension, compression, or shear. Kowalczyk-Gajewska et al. (2015) performed a crystal-plasticity analysis of equal-channel angular pressing and extrusion with a forward-backward rotating die of CP-Ti. They studied mainly the texture evolution during the processes but hardly investigated the work-hardening behavior. It should also be mentioned that material parameter identification is difficult for CP-Ti because of the large number of active deformation modes. For instance, the present authors (2016) reported that the difficulty in parameter identification arose also in $\mathrm{Mg}$ alloy sheets with smaller numbers of active deformation modes and that it was crucial to determine the parameters on the basis of the role of each active deformation mode. However, to the best of our knowledge, a systematic procedure of parameter identification has not been studied yet.

This study aimed at understanding the deformation mechanism upon different strain paths in a CP-Ti Grade 1 sheet using a crystal-plasticity finite-element method (FEM). First, material parameters were carefully identified considering the role of each active deformation mode. The deformation behavior under a variety of loading paths was then predicted by means of a crystal-plasticity FEM with the identified material parameters. Finally, the mechanism that yielded anisotropic deformation behaviors under various strain paths was investigated numerically.

\section{Experimental}

The experimental results reported in our previous study [Yi et al., 2016] were utilized to validate simulation results in the present study. However, some new experimental results were also used; thus, the experimental procedure is described briefly here. A cold-rolled CP-Ti JIS Grade 1 sheet with a thickness of $1 \mathrm{~mm}$ (Kobe steel) was used. Monotonic tension, monotonic compression, and reverse loadings from tension to compression (TC loading) and from compression to tension (CT loading) were conducted. To investigate the anisotropic deformation behavior, samples prepared along the RD and TD were used. The samples were annealed at $530^{\circ} \mathrm{C}$ for $1 \mathrm{~h}$ before the experiment. A through-thickness compressive stress of 3.6 MPa, which was less than 
$2.5 \%$ of the $0.2 \%$ proof stress in the $\mathrm{RD}$, was applied through a pair of comb-shaped dies to prevent buckling during compression. Solid lubricant (Moly Paste, Sumico Lubricant) was utilized as a lubricant to reduce the friction between the sample and comb-shaped dies. A uniaxial strain gage (KFEM, Kyowa Electronic Instruments) was employed for the measurement of strains. The tensile properties in the RD and TD are shown in Table 1.

The texture evolution on the ND-TD plane was measured using EBSD. EBSD measurements were undertaken at 500-fold magnification with a step size of $1.0 \mu \mathrm{m}$. OIM-Analysis 7 (TSL Solutions) was used to analyze the pole figures. To increase the accuracy of the results, measured data were omitted if a confidence index was smaller than 0.1 .

\section{Formulation of crystal-plasticity model for CP-Ti}

\subsection{Slip rate}

A crystal plasticity model employed in our previous studies predicted deformation behavior of magnesium alloy sheets upon various strain paths well [Hama and Takuda, 2011, 2012a, 2012b; Hama et al., 2013, 2015, 2016]. Therefore, the crystal-plasticity FEM used in the present study is basically the same as that used in the previous studies. The constitutive equation of a single crystal is described in Appendix A. A visco-plastic power law is given for the slip rate, $\dot{\gamma}^{(\alpha)}$, of the $\alpha$ slip system in the following form:

$\frac{\dot{\gamma}^{(\alpha)}}{\gamma_{0}}=\left|\frac{\tau^{(\alpha)}}{\tau_{\mathrm{Y}}^{(\alpha)}}\right|^{\frac{1}{m}} \operatorname{sign}\left(\tau^{(\alpha)}\right), \quad \dot{\tau}_{\mathrm{Y}}{ }^{(\alpha)}=\sum_{\beta} q_{\alpha \beta} h\left|\dot{\gamma}^{(\beta)}\right|, \quad \tau^{(\alpha)}=\boldsymbol{\sigma}:\left(\boldsymbol{s}^{(\alpha)} \otimes \boldsymbol{m}^{(\alpha)}\right)$

where $\tau^{(\alpha)}$ and $\tau_{Y}^{(\alpha)}$ denote respectively the resolved shear stress and the current strength of the $\alpha$ slip system. $\dot{\gamma_{0}}$ and $m$ are respectively the reference-strain rate and the rate-sensitivity exponent. $\boldsymbol{\sigma}$ is the Cauchy stress tensor, and $\boldsymbol{s}^{(\alpha)}$ and $\boldsymbol{m}^{(\alpha)}$ are the unit vectors that characterize the slip direction and the slip plane normal of the $\alpha$ slip system, respectively. $q_{\alpha \beta}$ represents the interaction matrix. $h$ is the hardening modulus, which is given in the forms [Graff et al., 2007]

$h=h_{0}$,

$h=h_{0}\left(1-\frac{\tau_{0}}{\tau_{\infty}}\right) \exp \left(-\frac{h_{0} \bar{\gamma}}{\tau_{\infty}}\right)$

where $\tau_{0}$ denotes the initial critical resolved shear stress, i.e., the initial value of $\tau_{Y}^{(\alpha)}$, and $h_{0}$ and $\tau_{\infty}$ are the hardening parameters. $\bar{\gamma}$ is given by $\bar{\gamma}=\sum_{\alpha} \int\left|\dot{\gamma}^{(\alpha)}\right| \mathrm{d} t$. It should be noted that it might be possible to reproduce experimental results using solely 
eq. (3). However, to simplify the problem, eq. (2) was also utilized for the twinning systems because work hardening was assumed to be small; thus, the number of parameters could be reduced. Note that the summation in eq.(1) is taken over all slip and twinning systems.

\subsection{Slip and twinning systems}

Following Benmhenni et al. (2013), five families of slip systems - three basal $\{0001\}<11 \overline{2} 0>$ slip, three prismatic $\{10 \overline{1} 0\}<11 \overline{2} 0>$ slip, three pyramidal $\{10 \overline{1} 1\}<11 \overline{2} 0>$ slip, six pyramidal $\{11 \overline{2} 2\}<11 \overline{2} 3>$ slip (pyramidal $<a+c>-1$ slip), and twelve pyramidal $\{10 \overline{1} 1\}<11 \overline{2} 3>$ slip (pyramidal $<a+c>-2$ slip) —and two families of twinning systems- six $\{10 \overline{1} 2\}$ tensile twinning and six $\{11 \overline{2} 2\}$ compressive twinning-were taken into consideration. As explained above, linear hardening [Eq. (2)] was used for the twinning systems and Voce hardening [Eq. (3)] was applied to the slip systems.

\subsection{Twinning and detwinning model}

A twinning and detwinning model employed in the present study [Hama and Takuda, 2012a] was the extension of the twinning model by Van Houtte (1978). In this model, the activity of the $\alpha$-twinning system is represented using the resolved shear stress, $\tau^{(\alpha)}$, as follows. This model assumes that $\{10 \overline{1} 2\}$ tensile twinning and $\{11 \overline{2} 2\}$ compressive twinning are active only when the resolve shear stresses are positive $\left(\tau^{(\alpha)}>0\right)$ and negative $\left(\tau^{(\alpha)}<0\right)$, respectively. Because the shear strain due to twinning activity can be considered to be mechanically similar to that due to slip activity, the shear strain rate due to twinning and detwinning is described using Eqs. (1) and (2).

The twin rotation is modeled as follows. If the shear in a grain is assumed to be uniform, the volume fraction of the $\alpha$-twinning system, $f^{(\alpha)}$, can be given by $f^{(\alpha)}=$ $\frac{\bar{\gamma}_{\text {twin }}^{(\alpha)}}{\gamma_{\text {ref }}}$, where $\bar{\gamma}_{\text {twin }}^{(\alpha)}$ and $\gamma_{\text {ref }}$ are the accumulated shear strain induced by twinning and the reference shear strain, respectively. When $f^{(\alpha)} \geq f_{\mathrm{th}}^{(\alpha)}$ is satisfied, where $f_{\mathrm{th}}^{(\alpha)}$ is the threshold value for the volume fraction, the orientation of each slip system is rotated due to the twinning activity using the rotation tensor, $\boldsymbol{R}^{t w}$, in the form:

$$
\boldsymbol{R}^{t w}=2 \boldsymbol{m}^{(\alpha)} \otimes \boldsymbol{m}^{(\alpha)}-\boldsymbol{I},
$$

where $\boldsymbol{I}$ represents the identity tensor. The threshold value $f_{\mathrm{th}}^{(\alpha)}$ is determined randomly to be in the range from 0.1 to 1.0 for each twinning system prior to the simulation. It 
should be noted that this range was also an additional parameter that was determined so as to reproduce experimental results as explained in the following sections. In the present model, the orientations of twinning system are not rotated even when the abovementioned condition is satisfied because the twinning systems are assumed to represent twin planes of matrix region. After the twin rotation takes place, the shear strain rate due to twinning cannot generate in the grain.

The present model assumes that $\{10 \overline{1} 2\}$ detwinning can be active when the sign of $\tau^{(\alpha)}$ is inverted from positive to negative, whereas $\{11 \overline{2} 2\}$ detwinning is active when the sign of $\tau^{(\alpha)}$ changes from negative to positive. Detwinning can be active until $\bar{\gamma}_{\text {ut }}^{(\alpha)}=\bar{\gamma}_{\text {twin }}^{(\alpha) \text { max }}$ is fulfilled, where $\bar{\gamma}_{\text {ut }}^{(\alpha)}$ and $\bar{\gamma}_{\text {twin }}^{(\alpha) \max }$ denote respectively the accumulated shear strain induced by detwinning and the accumulated shear strain induced by twinning that occurred before the sign of $\tau^{(\alpha)}$ is inverted. This assumption represents that detwinning can be active until the volume fraction of the $\alpha$-twinning system vanishes. If $f^{(\alpha)} \geq f_{\text {th }}^{(\alpha)}$ was satisfied and the slip systems were subjected to twin rotation during twinning before the sign of $\tau^{(\alpha)}$ was inverted, the slip systems are re-rotated using the rotation tensor $\left(\boldsymbol{R}^{\mathrm{tw}}\right)^{\mathrm{T}}$ when $\bar{\gamma}_{\mathrm{ut}}^{(\alpha)}=\bar{\gamma}_{\mathrm{twin}}^{(\alpha) \max }$ is fulfilled. The shear strain rate due to detwinning cannot generate in the grain after $\bar{\gamma}_{\mathrm{ut}}^{(\alpha)}=\bar{\gamma}_{\mathrm{twin}}^{(\alpha) \max }$ is fulfilled.

EBSD measurements performed in our previous experiment [Yi et al., 2016] showed that the number of grains in which both $\{10 \overline{1} 2\}$ tensile twinning and $\{11 \overline{2} 2\}$ compressive twinning were active simultaneously was less than $5 \%$ of the total number of grains. Moreover, the interaction between tensile and compressive twinning systems in these grains was negligible, irrespective of strain path. These experimental results indicated that the effect of the interaction between the two kinds of twinning on the macroscopic deformation behavior may be negligible. Therefore, because we focus our attention rather on macroscopic deformation behavior, it is assumed that either $\{10 \overline{1} 2\}$ tensile twinning or $\{11 \overline{2} 2\}$ compressive twinning can be active in each grain, i.e., the two kinds of twinning systems cannot be activated simultaneously in a grain.

\subsection{Procedure of finite-element simulation}

The aforementioned crystal-plasticity model was introduced into a static FEM with explicit time integration. To prevent a rapid increase in the nonequilibrated forces due to the explicit time integration, the generalized $r_{\min }$ strategy [Yamada et al., 1968] and the rate tangent modulus method [Pierce et al., 1984] were utilized. In particular, the generalized $r_{\min }$ strategy is effective to minimize the effect of unstable calculation due to 
twinning and detwinning rotations.

Regarding the finite-element model, a cube was divided uniformly into seven trilinear hexahedral elements in each direction, as shown in Fig. 1. The length of each side of the cubic was $10 \mathrm{~mm}$; thus, the length of each side of the finite element was approximately $1.43 \mathrm{~mm}$. All integration points in each element have an identical initial crystallographic orientation; therefore, the number of initial orientations in the finite-element model was $7 \times 7 \times 7=343$. The initial crystallographic orientations in each element were randomly selected from the results of EBSD measurements [Yi et al., 2016]. The pole figures of the virgin sheet obtained from the experiment are depicted in Fig. 2 (a). This number of finite elements, which is in fact rather small, was determined on the basis of the balance between the simulation accuracy and the computation time. The validity of this choice will be discussed later.

Concerning the boundary conditions assigned to the model (Fig. 1), the plane symmetry was assumed for the planes $x=0, y=0$, and $z=0$ and traction-free boundary conditions were imposed to the planes $y=l$ and $z=l$. When uniaxial loading, such as monotonic tension, monotonic compression, and reverse loading, was simulated, displacement increments were assigned to the plane $x=l$. Note that the $x$ direction was set to be the RD for the RD loading, whereas it was set to be the TD for the TD loading. In either case, the $z$ axis was defined to be the ND. On the other hand, when the deformation behavior during biaxial tension was simulated, the $x$ and $y$ directions were set to be respectively the RD and TD, and displacement increments were imposed to both planes $x=l$ and $y=l$ such that a pseudo-linear stress path under a prescribed stress ratio could be achieved [Hama and Takuda, 2012b]. The detail procedure is described in Appendix B.

\subsection{Determination of material parameters}

Isotropic elasticity was assumed in the present simulation because elastic anisotropy is not pronounced in CP-Ti. Following previous experiment [Yi, et al., 2016; Kuchinomachi, 2014], Young's modulus, E, Poisson's ratio, v, and the rate-sensitivity exponent, $m$, were set to be $E=105 \mathrm{GPa}, v=0.34$, and $m=0.02$, respectively. The reference-strain rate, $\dot{\gamma}_{0}$, was taken to be $\dot{\gamma}_{0}=0.001 \mathrm{~s}^{-1}$. The self-hardening parameters of $q_{\alpha \beta}$ were set to be 1 . Because the latent-hardening parameters of CP-Ti have rarely been examined in detail in previous studies, the following assumptions were made in the present study. On the basis of a previous study on magnesium alloys [Agnew et al., 2001], the latent-hardening parameters were assumed to be larger 
between the slip and twinning systems than between the slip systems. This assumption reflects the fact that the twinning activity yields large work hardening as reported in the literature [Bouaziz and Guelton, 2001; Ishiyama, 2006; Bouaziz et al., 2008; Nixon et al., 2010]. In addition, the parameters between pyramidal $\langle a+c\rangle$ slip and $\{11 \overline{2} 2\}$ twinning were assumed to be the largest. This assumption resulted from the parameter identification explained below. The interactions between the other slip systems were assumed to be independent of the slip system; that is, the same parameter was used irrespective of the combination of slip systems. Table 2 shows the resultant latent-hardening parameters, $q_{\alpha \beta}$, determined in the present study.

To identify the hardening parameters in Eqs. (2) and (3), the magnitude relation of CRSSs for each slip and twinning system was determined first. The CRSSs for slip and twinning systems at room temperature were investigated in previous studies [Conrad, 1981; Philippe et al., 1995; Wu et al., 2007; Warwick et al., 2012; Benmhenni et al., 2013] as follows. Warwick et al. (2012) reported that the deformation mode with the smallest CRSS was prismatic slip, followed in order by basal slip, pyramidal $<a>$ slip, $\{10 \overline{1} 2\}$ twinning, $\{11 \overline{2} 2\}$ twinning, and pyramidal $\langle a+c>$ slip. Philippe et al. (1995) reached a similar conclusion. Wu et al. (2007) reported that the deformation mode with the smallest CRSS was prismatic slip, followed in order by pyramidal $\langle a+c\rangle$ slip, twinning, and basal slip. Conrad (1981) studied the order of CRSSs experimentally and reported that the smallest CRSS was prismatic slip, followed in order by $\{10 \overline{1} 2\}$ twinning, $\{11 \overline{2} 2\}$ twinning, and basal slip. It is thus reasonable to presume that the deformation mode with the smallest CRSS is prismatic slip, followed in order by pyramidal $<a>$ slip, $\{10 \overline{1} 2\}$ twinning, $\{11 \overline{2} 2\}$ twinning, and pyramidal $\langle a+c\rangle$ slip. In contrast, the rank of the CRSS for basal slip is still open to discussion because it is different depending on the researcher. Therefore, in this study, the CRSS for basal slip was adjusted to reproduce experimental results accurately while keeping the abovementioned magnitude relation of CRSSs for slip and twinning systems unchanged.

The hardening parameters for slip and twinning systems were then calibrated. To identify the hardening parameters considering the role of each slip and twinning system on the work-hardening behavior, the strain-path dependency of active slip and twinning systems was taken into account as follows. Roth et al. (2014) examined active slip systems under tension in terms of Schmid's factor and CRSS for each slip system. They reported that prismatic slip was primarily active at the beginning of plastic deformation in the RD. They also found that, in the RD, pyramidal $<a>$ slip was active as well, whereas basal slip was hardly active. On the other hand, in the TD, they showed that the activity of pyramidal $<a>$ slip might be initially dominant, and prismatic slip and basal 
$<a>$ slip were also active. The active twinning systems under monotonic compression and monotonic tension in the RD were also studied by the present authors [Hama et al., 2014; Yi et al., 2016]: $\{10 \overline{1} 2\}$ twinning was active under compression, whereas $\{11 \overline{2} 2\}$ twinning was active under tension. Based on the abovementioned results, the hardening parameters for the slip and twinning systems were systematically determined using the following four steps. Note that a strain path where pyramidal $\langle a+c\rangle$ slip is primarily active is unknown because of its high CRSS; therefore, the hardening parameters for pyramidal $\langle a+c\rangle$ slip were determined based only on the information that the CRSS of pyramidal $\langle a+c\rangle$ slip was more than twice as large as that for prismatic slip [Warwick et al., 2012; Paton and Backofen, 1970; Zambaldi et al., 2012; Gong and Wilkinson, 2009].

(1) The hardening parameters reported by Warwick et al. (2012) were utilized as the initial value for the following iteration, where the CRSS of pyramidal $\langle a+c\rangle$ slip was set to be approximately three times larger than that for prismatic slip.

(2) The hardening parameters for prismatic slip and $\{11 \overline{2} 2\}$ twinning were primarily estimated to fit the stress-strain curve, the evolution of the $r$ value, and the texture evolution obtained under monotonic tension in the RD. Similarly, the stressstrain curve under TC loading in the $\mathrm{RD}$ was used to adjust the hardening parameters for $\{11 \overline{2} 2\}$ detwinning.

(3) The parameters for pyramidal $<a>$ and basal slip were primarily estimated by referring to the stress-strain curve and the evolution of the $r$ value obtained under monotonic tension in the TD.

(4) The parameters for $\{10 \overline{1} 2\}$ twinning were primarily estimated from the texture evolution and the stress-strain curve obtained under compression in the RD. The stressstrain curve under CT loading in the RD was utilized to adjust the hardening parameters for $\{10 \overline{1} 2\}$ detwinning.

Steps (2), (3), and (4) were repeated several times to achieve a good fit with all the experimental results using a single set of parameters.

The determined hardening parameters for each slip and twinning system are given in Table 3. The parameter determination for the twinning and detwinning model is additionally explained. As mentioned earlier, the range of the threshold value for the volume fraction $f_{\mathrm{th}}^{(\alpha)}$ was determined to be from 0.1 to 1.0 using the abovementioned procedure. As in the case of initial crystallographic orientations, a same value was assigned for $f_{\text {th }}^{(\alpha)}$ to all eight integration points in each element. The primal role of this 
range and the hardening parameters for twinning is to reproduce macroscopic stress-strain curves and evolutions of texture and volume fraction of twin. On the other hand, it would be reasonable to assume that the reference shear strain $\gamma_{\text {ref }}$ corresponds to the twinning shear strains of approximately 0.176 and 0.217 for $\{10 \overline{1} 2\}$ twinning and $\{1 \overline{2} 2\}$ twinning, respectively [Christian and Mahajan, 1995; Battaini et al., 2007]. Because the hardening parameters, the reference shear strains, and the range of the threshold values for the volume fraction are strongly connected with each other, it was assumed from a mesoscopic viewpoint that the reference shear strain was not limited exactly to these specific values but could be subjected to a slight change from these values. As a result, the reference shear strain was adjusted to be $\gamma_{\text {ref }}=0.21$ for $\{10 \overline{1} 2\}$ twinning and $\gamma_{\text {ref }}=0.27$ for $\{11 \overline{2} 2\}$ twinning.

The proposed procedure would be useful for determining systematically the material parameters for any rolled CP-Ti sheet. On the other hand, it should also be noted that we cannot guarantee that the determined parameters are most suitable for the present experimental results because the parameters were determined rather empirically. For instance, we cannot exclude the possibility that the parameters are determined satisfactorily while keeping the reference shear strains to be the exact values of twinning shear strains. It is also difficult to validate the latent-hardening parameters shown in Table 3. Therefore, further effort is still necessary to validate the parameters from a physical viewpoint.

\section{Simulation results}

\subsection{Effect of number of initial orientations on simulation result}

The purpose of section 4 is to verify the simulation results through comparison with experimental results reported in Yi et al. (2016). Before going into detail, the effect of the number of initial orientations on a simulation result is discussed. A simulation of uniaxial tension was conducted with five different sets of initial crystallographic orientations using a finite-element model with 125 elements $(5 \times 5 \times 5)$, as well as a model with 343 elements (Fig. 1). Figs. 2 (b) and (c) show typical examples of the pole figures obtained from the initial crystallographic orientations used in the simulation respectively for finite-element models with 343 and 125 elements. The pole figures for the model with 343 elements reproduce well those of the experiment (Fig. 2 (a)), whereas those for the model with 125 elements are different notably from those of the experiment. Note that the pole figures remain almost unchanged irrespective of the set 
of crystallographic orientations for the model with 343 elements, whereas they are significantly different depending on the set of orientations for the model with 125 elements. Fig. 3 shows the stress-strain curves under uniaxial tension in the RD obtained for the models with 125 and 343 elements. Cauchy stress and Green-Lagrange strain tensors that were averaged over the domain were used as true stress and strain in the simulation results. The Green-Lagrange strain was used because a same definition of strain can be employed for any strain components. On the other hand, it should also be noted that the difference between the logarithmic and Green-Lagrange strains would not be negligible at large strains. The stress-strain curves obtained with the model with 125 elements are slightly different depending on the set of initial orientations, whereas those of the model with 343 elements are almost independent of the set of initial orientations, at least within the strain range tested. These results demonstrate that the present simulation model would be reasonable to predict the deformation behavior of the polycrystalline CP-Ti Grade 1 sheet.

\subsection{Monotonic tension and compression}

Fig. 4 shows the absolute stress-absolute strain curves under monotonic tension and compression. In the following figures, true stress and logarithmic strain were used for the experimental results. Fig. 5 displays the evolution of the work-hardening rate. The following trends observed in the experimental results were predicted qualitatively well in the simulation results: (1) The yield stress in the RD was smaller than that in the $\mathrm{TD}$, whereas the work hardening in the RD was slightly larger than that in the TD. This trend was the same in tension and compression [Ishiki et al., 2009, 2011; Roth et al., 2014; Ishiyma et al., 1990]. (2) For the RD loading, the yield stress was larger under tension than under compression, whereas the work hardening was smaller under tension than under compression. In contrast, for the TD loading, the yield stresses in tension and compression were approximately the same, whereas the work hardening was smaller under tension than under compression [Nixon et al., 2010].

Fig. 6 shows the evolution of the $r$ value obtained under monotonic tension. The normal components of Green-Lagrange strain tensors averaged over the domain were used to calculate the $r$ value in the simulation. In the experiment [Ishiki et al., 2009, 2011; Yi et al., 2016], the $r$ value was significantly larger in the TD than in the RD, regardless of the amount of plastic strain. Moreover, the $r$ value in the TD decreased rapidly at the beginning of the plastic deformation, whereas that in the RD remained almost unchanged. The simulation results captured the aforementioned evolution 
qualitatively well.

\subsection{TC loading}

Fig. 7 depicts the stress-strain curves under TC loading in the RD and TD. Figs. 8 and 9 display the variation of the work-hardening rate after the stress reversal for the $\mathrm{RD}$ and TD cases, respectively. Note that a tensile pre-strain of either 0.05 or 0.1 was given. In the $\mathrm{RD}$, the work-hardening rate remained almost unchanged during the earlier stage of compression, whereas it increased slightly during the latter stage. The aforementioned tendency was independent of the tensile pre-strain. On the other hand, in the TD, the work-hardening rate remained almost unchanged during compression, irrespective of the tensile pre-strain. The simulation results agreed fairly well with the experimental results.

Fig. 10 presents the pole figures for TC loading in the RD obtained at points $\mathrm{A}$ and B [Fig. 7(a)]. In the (0001) pole figure, weak peaks in the RD appeared at point A, while the intensity of the peaks in the RD increased at point B. Fig. 11 presents the pole figures for the TD case obtained at points $C$ and D [Fig. 7(b)]. In the (0001) pole figure, weak peaks in the $\mathrm{RD}$ appeared at point $\mathrm{C}$, as in the case of the $\mathrm{RD}$ loading. In contrast, the peaks in the RD did not appear at point $\mathrm{D}$. The aforementioned texture evolution was well reproduced in the simulation.

\subsection{CT loading}

Fig. 12 shows the stress-strain curves under CT loading in the RD and TD. Figs. 13 and 14 depict the variations of the work-hardening rate after the stress reversal for the $\mathrm{RD}$ and TD cases, respectively. Note that a strain given during compression was either $0.03,0.05,0.07$, or 0.1 in the RD case, whereas it was either 0.05 or 0.1 in the TD case. In the RD case of the experimental result [Fig. 12(a)], the slope of the curves changed rapidly and a small stress peak arose at a strain of approximately zero after the stress reversal, regardless of the compressive pre-strain. This trend is also observed in Fig. 13(a). Fig. 15 presents the relationship between the compressive pre-strain and the peak work-hardening rate, $g_{\mathrm{T}}$, which corresponds to the slope of the stress-strain curve just before the stress peak arises. The experimental results showed that $g_{\mathrm{T}}$ decreased rapidly with increasing the compressive pre-strain. The simulation results for the compressive pre-strains of $0.03,0.05$, and 0.07 were in fairly good agreement with the aforementioned experimental results, whereas a stress peak hardly occurred for the 
compressive pre-strain of 0.1 in the simulation result; thus, $g_{\mathrm{T}}$ could not be identified (Figs. 12 (a), 13 (b), and 15).

For the TD case, neither a stress peak [Fig. 12(b)] nor a rapid change in the work hardening (Fig. 14) appeared after the stress reversal in either the experimental or the simulation results.

Fig. 16 presents the pole figures for CT loading in the RD obtained at points E, F, G, and $\mathrm{H}$ shown in Fig. 12(a). In the (0001) pole figure, strong peaks in the RD occurred at point E [Fig. 16(a)]. As the tensile strain increased after the stress reversal, the peaks in the RD gradually disappeared [Figs. 16(b) and (c)], and then strong peaks in the RD arose again [Fig. 16(d)]. The texture evolution observed in the experiment was predicted well in the simulation results.

Fig. 17 presents the pole figures for the TD case obtained at points I and J shown in Fig. 12(b). The (0001) pole figure at points I and J [Figs. 17(a) and (b), respectively] were very close to the initial pole figure (Fig. 2) in the experiment, whereas weak peaks appeared in the RD after the stress reversal (point J) in the simulation [Fig. 17(b)]. These results indicate that the simulation results did not capture the features observed in the experimental results when the sheet was subjected to TC loading in the TD.

\subsection{Biaxial tension}

Fig. 18 shows the contours of plastic work measured at various uniaxial plastic strains in the RD, $\varepsilon_{0}^{p}$, obtained from the simulation. The contours were normalized by using the uniaxial stress in the $\mathrm{RD}, \sigma_{0}$. The following tendencies are observed in the evolution of the contours:

- The contour of plastic work exhibits strong asymmetry with respect to the line $\sigma_{x}=\sigma_{y}$ at the beginning of the plastic deformation.

- The contour approaches a symmetric shape with respect to the line $\sigma_{x}=\sigma_{y}$ as the plastic strain increases, indicating that the contour exhibits differential work hardening.

- Eventually, the contour results in an approximately symmetric shape at $\varepsilon_{0}^{p}=0.085$.

The variation of the shape ratio of the normalized contour as a function of $\varepsilon_{0}^{p}$ is shown in Fig. 19. Following Ishiki et al. (2009, 2011), the shape ratio is defined as $L / L_{0}$, where $L$ is the current distance from the origin to a stress point, and $L_{0}$ is the distance at $\varepsilon_{0}^{p}=0.085$. The shape ratio decreased rapidly immediately after the plastic deformation began, irrespective of the stress ratio. The change in the shape ratio was smaller for the stress ratios $\sigma_{x}: \sigma_{y}=1: 0,4: 1,2: 1$, and 4:3 than for the stress ratios 1:1, 
$3: 4,1: 2,1: 4$, and $0: 1$. In contrast, in the latter stage of the plastic deformation, the shape ratio decreased more gradually irrespective of the stress ratio. The abovementioned trends capture those observed in the experimental results [Ishiki et al., 2009, 2011] qualitatively well. Note that the material used in the experiment [Ishiki et al., 2009, 2011] was not the same as that used in the present study; thus, the comparison between the experimental and simulation results is carried out only qualitatively.

In conclusion, the present simulation results capture qualitatively well the in-plane anisotropy as well as tension-compression asymmetry observed in the experimental results, verifying the present crystal-plasticity model including the determined parameters.

\section{Discussion}

The mechanism that yielded the aforementioned anisotropies was investigated numerically in this section. The evolution of the relative activity was utilized for this purpose. The relative activity for each family, $i$, is given as [Tome et al., 1991]:

$r_{i}=\frac{\sum_{n_{s}}^{n} \sum_{\alpha}^{k}\left|\Delta \gamma^{\left(n_{s}, \alpha\right)}\right|}{\sum_{n_{s}}^{n} \sum_{\beta}^{N}\left|\Delta \gamma^{\left(n_{s}, \beta\right)}\right|}$

where $k$ is the number of slip or twinning systems of family $i, n$ is the number of grains, and $N$ is the total number of slip and twinning systems in a grain.

\subsection{In-plane anisotropy in stress-strain curve under monotonic tension}

Fig. 20 shows the evolution of relative activity during tension in the RD and TD. The activity of prismatic slip is dominant at the very beginning of the plastic deformation, and then the activity of pyramidal $<a>$ slip increases rapidly in both $\mathrm{RD}$ and TD. On the other hand, the initial increasing rate in the activity of pyramidal $\langle a\rangle$ slip and the magnitude of activity eventually reached are larger in the TD than in the RD. This result suggests that the dominant deformation mode at yielding is prismatic slip in the $\mathrm{RD}$, whereas it is pyramidal $<a>$ slip in the TD, consistent with the interpretation by Roth et al. (2014). This result also explains that the yield stress under tension in the RD is smaller than that in the TD because the CRSS of prismatic slip is smaller than that of pyramidal $<a>$ slip (Table 3 ).

After yielding, in the RD [Fig. 20(a)], the activities of prismatic slip and pyramidal 
$<a>$ slip are dominant, and $\{11 \overline{2} 2\}$ twinning is active as well. Two types of pyramidal $\langle a+c\rangle$ slip are also active to a small extent. On the other hand, in the TD [Fig. 20(b)], the activity of pyramidal $<a>$ slip is dominant, and basal slip and $\{10 \overline{1} 2\}$ twinning, as well as prismatic slip, are also active. Note that the predicted twinning activities are consistent with the results of EBSD measurement [Yi, et al., 2016]. These results suggest that the difference in both slip and twinning activities would result in the anisotropy in the work hardening as follows. The slip resistance is the smallest for prismatic slip and the largest for pyramidal $\langle a+c\rangle$ slip (see Table 3) among the active slip systems. Hence, the difference in the slip resistance between the slip systems activated at and after yielding is larger in the RD than in the TD; thus, larger work hardening is attained in the RD. On the other hand, the magnitude of the twinning activity is similar in the RD and TD in the initial stage, whereas it is larger in the RD than in the TD in the latter stage. This difference in the twinning activity would also influence the in-plane anisotropy in the work hardening in the latter stage of tension because the latent-hardening parameters were assumed to be larger between slip and twinning systems than between slip systems (Table 2).

To support these presumptions, Figs. 21 and 22 show the respective stress-strain curves and evolution of the work-hardening rate under monotonic tension obtained with and without considering twinning activity. The difference in the work hardening between the RD and TD apparently decreases at large strains when the twinning activity is not considered, but at the same time, the work-hardening rate in the TD is still slightly smaller than that in the RD. Clearly, the in-plane anisotropy in work hardening during tension is affected by the activities of both slip and twinning, verifying the aforementioned discussion.

\subsection{In-plane anisotropy in $r$ value under monotonic tension}

Because the strong basal texture is developed (Fig. 2), it is presumed that the activities of pyramidal $\langle a+c\rangle$ slip and twinning primarily contribute to the macroscopic thickness strain in both RD and TD. On the other hand, the activities of pyramidal $\langle a+c>-1$ and $\langle a+c>-2$ slip are far smaller than the twinning activity in both the RD and the TD (Fig. 20). Therefore, the twinning activity would play a dominant role in the thickness strain. Because of the polar character of twinning, the activity of $\{11 \overline{2} 2\}$ compressive twinning during tension in the $\mathrm{RD}$ would contribute to the decrease in the thickness, whereas the activity of $\{10 \overline{1} 2\}$ tensile twinning during tension in the TD would suppress the decrease in the thickness. This difference in the twinning activity 
could be responsible for the fact that the $r$ value in the TD is larger as compared to that in the RD.

On the other hand, it should also be noted that the evolution of the $r$ value in the TD would not be explained solely by twinning because, in the initial stage, the $r$ value decreases rapidly, whereas the twinning activity remains almost unchanged. It is presumed that, during tension in the TD, the thickness strain would also be affected by $<a>$ slip to some extent due to the fact that the $c$-axes tilt 20 to $40^{\circ}$ from the ND to the TD (Fig. 2).

\subsection{Deformation mechanism under biaxial tension}

Figs. 23 and 24 show the evolution of the relative activities for stress ratios $\sigma_{x}: \sigma_{y}$ ranging from 1:0 to $1: 1$ and from 3:4 to $0: 1$, respectively, as a function of plastic work. Note that we focus on the deformation behavior until the plastic work reaches approximately $3.8 \mathrm{MJ} / \mathrm{m}^{3}$, i.e., a uniaxial plastic strain of 0.02 , because the changes in the shape ratios of the contours are large in this deformation range, as shown in Fig. 19. Moreover, because the twinning activity is relatively small in this range, irrespective of stress ratio, the effect of the slip activities on the anisotropic deformation behavior is discussed.

The pyramidal $<a>$ slip activity increases as the stress ratio changes from 1:0 (uniaxial tension in the RD) to 1:1 (equibiaxial tension), and eventually, the activity of pyramidal $<a>$ slip is the largest among the active systems throughout the deformation for $\sigma_{x}: \sigma_{y}=4: 3$ and $1: 1$. The tendency that $\{11 \overline{2} 2\}$ twinning and one of pyramidal $<a+c>$ slip systems are active to a small extent after yielding is similar for stress ratios ranging from 1:0 to $1: 1$. In contrast, the pyramidal $<a>$ slip activity drops sharply and the basal slip activity increases as the stress ratio changes from 1:1 to 1:2. Eventually, the basal slip activity is the largest among the active systems for $\sigma_{x}: \sigma_{y}=3: 4$ and 1:2. The pyramidal $<a>$ slip activity then increases again and the basal slip activity decreases as the stress ratio approaches 0:1 (uniaxial tension in the TD). Pyramidal $<a+c>-2$ slip is also active for stress ratios $3: 4$ and $1: 2$, whereas $\{10 \overline{1} 2\}$ twinning is active for stress ratios $1: 2,1: 4$, and $0: 1$.

The change in the activities is large up to a plastic work of approximately $1 \mathrm{MJ} / \mathrm{m}^{3}$, irrespective of the stress ratio. In contrast, in the subsequent deformation, the activities change gradually in the range $\sigma_{x} \geq \sigma_{y}$, whereas in the range $\sigma_{x} \leq \sigma_{y}$ the activities remain almost unchanged.

On the basis of the aforementioned evolution, the asymmetry in the initial contour is 
examined first. The relationship between the relative activity and the biaxial stress ratio at a uniaxial plastic strain of 0.001 (Fig. 25) was used to analyze the overall trend of the change in the relative activity as a function of biaxial stress ratio. The plastic deformation at yielding is governed primarily by prismatic slip and pyramidal $<a>$ slip in the range $\sigma_{x} \geq \sigma_{y}$ as follows: the prismatic slip activity is the largest at $\sigma_{x}: \sigma_{y}=1: 0$ and decreases as the ratio approaches $1: 1$, whereas the pyramidal $<a>$ slip activity increases as the ratio approaches 1:1. In contrast, roughly speaking, the plastic deformation is governed by pyramidal $<a>$ slip and basal slip in the region $\sigma_{x}<\sigma_{y}$ as below: the pyramidal $\left\langle a>\right.$ slip activity is the largest at $\sigma_{x}: \sigma_{y}=0: 1$ and decreases as the stress ratio changes from $0: 1$ to $3: 4$. On the other hand, the basal slip activity increases as the stress ratio changes from $0: 1$ to $3: 4$ and is larger than the pyramidal $<a>$ slip activity for the stress ratios of 3:4 and 1:2. These results suggest that the initial contour of plastic work bulges more in the range $\sigma_{x} \leq \sigma_{y}$ than in the range $\sigma_{x} \geq \sigma_{y}$ because the CRSS of pyramidal $<a>$ slip is larger than that of prismatic slip. Moreover, the peak in the contour of plastic work occurs around the stress ratios of 3:4 and 1:2 because the activity of basal slip, whose CRSS is larger than that of pyramidal $<a>$ slip and prismatic slip, is the largest among the active systems at these stress ratios.

Next, the significant change in the shape of normalized contour in the range $\sigma_{x} \leq$ $\sigma_{y}$ is discussed. Because the contours are normalized by the uniaxial stress in the RD, $\sigma_{0}$, a comparison is made between the results for the stress ratio $\sigma_{x}: \sigma_{y}=1: 0$ and the other stress ratios. As explained earlier, the dominant active slip systems change from prismatic slip to pyramidal $<a>$ slip during uniaxial tension in the RD. Similar evolution of relative activity is presented for the stress ratios of $4: 1$ and 2:1; hence, the increase in flow stress would be pronounced as in the case of uniaxial tension in the RD. On the other hand, for the stress ratios ranging from $4: 3$ to $0: 1$, the dominant active slip remains unchanged after yielding; thus, the increase in flow stress is less pronounced. Therefore, the difference between the flow stress at $\sigma_{x}: \sigma_{y}=1: 0$ and that in the range $\sigma_{x} \leq \sigma_{y}$ decreases as the plastic work increases; thus, the normalized contour shrinks in the range $\sigma_{x} \leq \sigma_{y}$ profoundly.

\subsection{In-plane anisotropy under monotonic compression}

Fig. 26 shows the evolution of relative activities obtained under compression in the $\mathrm{RD}$ and TD. The dominant slip system at yielding is different between the RD and TD: the activities of prismatic slip and pyramidal $<a>$ slip are dominant in the RD and TD, respectively. As in the case of tension, this difference would result in the fact that the 
yield stress under compression is smaller in the RD than in the TD.

The reason why the work hardening is different between the RD and TD could be explained from the difference in the slip and twinning activities after yielding between the RD and TD. After yielding, pyramidal $<a>$ slip and $\{10 \overline{1} 2\}$ twinning become activated as well as prismatic slip for the RD loading [Fig. 26(a)]. In contrast, for the TD loading, basal slip and pyramidal $\langle a+c\rangle-2$ slip become activated in addition to pyramidal $<a>$ slip [Fig. 26(b)]. $\{11 \overline{2} 2\}$ twinning is also active, but the activity is much smaller than that of $\{10 \overline{1} 2\}$ twinning for the RD loading. Note that the predicted twinning activities are consistent with the results of EBSD measurement [Yi, et al., 2016].

The effect of the difference in the twinning activity on the difference in the work hardening can be investigated from the simulation results without considering twinning (Figs. 21 and 22). The evolution of the work hardening is approximately the same in the $\mathrm{RD}$ and TD when the twinning activity is not considered. This result suggests that the difference in the twinning activity would be the key factor for the in-plane anisotropy in the work hardening under compression. This result verifies the discussion presented in the literature [Nixon et al., 2010; Ishiyama, 2006; Bouaziz and Guelton, 2001; Bouaziz et al., 2008].

\subsection{Tension-compression asymmetry under monotonic loading}

First, the tension-compression asymmetry in the $\mathrm{RD}$ is discussed. Comparing the relative activities between tension [Fig. 20 (a)] and compression [Fig. 26 (a)] in the RD, the slip activities are very similar in tension and compression, whereas the $\{11 \overline{2} 2\}$ twinning activity under tension is much smaller as compared to the $\{10 \overline{1} 2\}$ twinning activity under compression. The difference observed in the twinning activities is in good agreement with the result of EBSD measurement [Yi, et al., 2016]. These results suggest that the difference in the twinning activities is one of the factors that yield the asymmetry in the stress-strain curve, as also presented by Yi et al. (2016). This presumption can be confirmed from the simulation results without considering twinning (Figs. 21 and 22): The difference in the rate of work-hardening, as well as the yield stress, between tension and compression drastically decreases when the twinning activity is not considered.

On the other hand, the mechanism for the TD loading is different from that of the RD loading as follows. The activities of pyramidal $<a>$ slip, prismatic slip, and basal slip are very similar in tension [Fig. 20 (b)] and compression [Fig. 26 (b)] at yielding, 
whereas the activity of pyramidal $\langle a+c\rangle-2$ slip is larger under compression than under tension and the $\{10 \overline{1} 2\}$ twinning activity under tension is slightly larger than the $\{1 \overline{2} 2\}$ twinning activity under compression. This result shows that the agreement in the yield stresses between tension and compression would be rather by chance because the slip and twinning activities at yielding are apparently different.

After yielding, the decrease in the activity of pyramidal $<a>$ slip and the increase in the activity of basal slip are more pronounced under compression than under tension. Moreover, the activity of $\{10 \overline{1} 2\}$ twinning under tension is larger than that of $\{11 \overline{2} 2\}$ twinning under compression. This difference in the twinning activities is consistent with the result of EBSD measurement (Yi, et al., 2106). The aforementioned results suggest that the larger work hardening under compression results primarily from the fact that the increase in the activity of basal slip, whose slip resistance is larger than that of pyramidal $<a>$ slip (see Table 3 ), is more pronounced under compression as compared to under tension. In contrast, the effect of the difference in the twinning activities would be negligible because the difference in the work hardening remained almost unchanged regardless of twinning activity, as shown in Fig. 22.

\subsection{Deformation mechanism under TC loading}

Regarding TC loading in the RD, Fig. 27(a) shows the evolution of the relative activity as a function of accumulated absolute strain in the case of a tensile pre-strain of 0.1 in the RD. After the stress reversal, $\{101 \overline{2}\}$ twinning is active in the initial stage, as in the case of monotonic compression [Fig. 26(a)] and, at the same time, $\{11 \overline{2} 2\}$ detwinning is also active to a small extent. Note that these twinning activities could also be observed in the results of EBSD measurement [Yi, et al., 2016]. The activities of the two kinds of pyramidal $\langle a+c\rangle$ slip and basal slip are initially small, but they increase in the latter stage and are larger than the $\{101 \overline{2}\}$ twinning activity at strains larger than approximately 0.23 . The abovementioned transition suggests that the work hardening increases in the latter stage after the stress reversal [Fig. 7(a)] because the deformation modes with high CRSS are pronounced only in the latter stage. Note that this trend is much less pronounced under monotonic compression, as presented in Figs. 4, 5, and 26. This would be because the monotonic compression test was conducted only up to a strain of 0.1 . Therefore, the work-hardening rate would increase if the compression test was done at larger strains. Nixon et al. (2010) conducted a monotonic compression test in the $\mathrm{RD}$ up to a strain of 0.4 and reported that the work-hardening rate was larger at 
large strains, which is consistent with this presumption.

The texture evolution in the (0001) pole figure during TC loading in the RD can also be explained using the relative activity. The weak peaks that appeared in the RD under tension [Fig. 10(a)] would be due to the small activity of $\{11 \overline{2} 2\}$ twinning [Fig. 27(a)]. After the stress reversal, the activity of $\{10 \overline{1} 2\}$ twinning is larger than that of $\{11 \overline{2} 2\}$ detwinning [Fig. 27(a)], leading to the increase in the intensity of the peaks [Fig. 10(b)]. These results are consistent with the experimental observations [Yi et al., 2016].

As for TC loading in the TD [Fig. 27(b)], the activity of $\{10 \overline{1} 2\}$ detwinning is comparatively large in the initial stage after the stress reversal, and the $\{11 \overline{2} 2\}$ twinning and pyramidal $\langle a+c\rangle$ slip activities increase as the detwinning activity is completed in the latter stage. This result shows that the pre-compression somewhat affects the deformation after the stress reversal. In contrast, the activities of basal slip and pyramidal $<a>$ slip are dominant throughout the process, as in the case of monotonic compression in the TD [Fig. 26(b)]; thus, the significant change in work hardening does not occur after the loading direction was reversed [Fig. 7(b)].

The texture evolution in the (0001) pole figure during TC loading in the TD could be described in terms of the twinning and detwinning activities. The weak peaks that appeared in the RD under tension [Fig. 11(a)] could be attributed to the small activity of $\{10 \overline{1} 2\}$ twinning [Fig. 27(b)]. After the stress reversal, $\{10 \overline{1} 2\}$ detwinning activity is almost completed at a strain of approximately 0.2. Moreover, $\{10 \overline{1} 2\}$ and $\{11 \overline{2} 2\}$ twinning activities are small during tension; thus, the weak peaks eventually disappear at the end of the deformation [Fig. 11(b)].

\subsection{Deformation mechanism under CT loading}

First, the reason why the rapid changes in work-hardening rate arise in the case of the RD is studied. Fig. 28 shows the evolution of relative activity with compressive pre-strains of 0.03 and 0.1 in the RD. When a compressive pre-strain of 0.03 was given [Fig. 28(a)], the activity of $\{10 \overline{1} 2\}$ detwinning is pronounced, and pyramidal $\langle a>$ and prismatic slip are also active at the beginning after the stress reversal. The activity of $\{10 \overline{1} 2\}$ detwinning then decreases sharply, and when it saturates to zero at an accumulated strain of approximately 0.06, $\{11 \overline{2} 2\}$ twinning and pyramidal $\langle a+c\rangle$ slip systems become activated rapidly. Apparently, the active deformation modes are different between the initial and latter stages after the stress reversal, and the transition of active deformation modes is rapid. The results of EBSD measurement [Yi et al., 2016] support these twinning activities. It should be noted that the sigmoidal curve 
occurs in the stress-strain curve at an accumulated strain of approximately 0.06 (Fig. 12). The aforementioned evolution shows that the work-hardening rate changes rapidly because the active deformation modes change rapidly to $\{11 \overline{2} 2\}$ twinning and pyramidal $\langle a+c\rangle$ slip systems whose slip resistances are far larger than the slip resistance of $\{10 \overline{1} 2\}$ detwinning. This conclusion supports the presumption by the present authors [Yi et al., 2016] that was discussed on the basis of experimental results. This result further indicates that this mechanism is very similar to that which yields a sigmoidal curve upon CT loading in Mg alloy sheets [Hama and Takuda, 2012a].

The reason why the peak work-hardening rate, $g_{T}$, decreases as the compressive pre-strain increases in the RD (Fig. 15) can also be explained from the viewpoint of the twinning activity. The decreasing rate of $\{10 \overline{1} 2\}$ detwinning activity in the earlier stage and the increasing rates of the $\{11 \overline{2} 2\}$ twinning and two types of pyramidal $\langle a+c>$ slip activities in the latter stage are much smaller for the compressive pre-strain of 0.1 [Fig. 28(b)] as compared to that for the compressive pre-strain of 0.03 ; thus, the change in the work-hardening rate in the vicinity of the sigmoidal curve also decreases.

The evolution of textures in the (0001) pole figure during CT loading in the RD (Fig. 16) can also be explained with respect to the twinning activity. The strong peaks that arise in the RD upon compression [Fig. 16(a)] could be due to the large activity of $\{10 \overline{1} 2\}$ twinning [Fig. 28(b)]. After the stress reversal, $\{10 \overline{1} 2\}$ detwinning activity leads to the decrease in the intensity of the peaks with increasing tensile strain. In contrast, the strong peaks arise again in the $\mathrm{RD}$ because $\{11 \overline{2} 2\}$ twinning is active after the $\{10 \overline{1} 2\}$ detwinning activity is almost completed. These results are consistent with the experimental observations using EBSD [Yi et al., 2016].

On the other hand, the evolution of relative activity in the TD case (Fig. 29) is notably different from those of the $\mathrm{RD}$ case; the rapid transition in the active deformation modes does not occur regardless of the compressive pre-strain. This is because the detwinning activity in the TD is much less pronounced as compared to that in the RD owing to the smaller twinning activity before the stress reversal and because the detwinning activity decreases not rapidly but gradually with the plastic deformation. Similarly, a clear evolution of texture does not occur during CT loading in the TD (Fig. 17) because the activities of twinning and detwinning in the TD are much less pronounced than those in the $\mathrm{RD}$. As described earlier, weak peaks appeared in the $\mathrm{RD}$ in the (0001) pole figure after the stress reversal only in the simulation results [Fig. 17(b)]. This would be because the $\{10 \overline{1} 2\}$ twinning activity after the stress reversal is larger than in reality. 


\section{Conclusion}

The deformation behavior upon various strain paths in a CP-Ti Grade 1 sheet was examined by means of a crystal-plasticity finite-element method. After the material parameters were systematically determined considering the role of each active deformation mode, the mechanism that yielded the anisotropic deformation behavior upon different strain paths was investigated numerically. The following conclusions were obtained from this work:

1. The simulation results were in fairly good agreement with the experimental results for all strain paths tested in this study, verifying the present crystal plasticity model as well as the determined material parameters.

2. The difference in the dominant active slip systems depending on the stress path played a crucial role in the anisotropy in the yield stress upon uniaxial tension, and in the wider sense, the asymmetry in the initial plastic contour and the differential work hardening upon biaxial tension at small strains. In contrast, twinning activities also affected the anisotropies in the work hardening at large strains and the $r$ value under uniaxial tension.

3. During compression, the difference in the dominant active slip systems played an important role in the in-plane anisotropy in the yield stress, as in the case of tension. On the other hand, the difference in the twinning activity would be a key factor for the in-plane anisotropy in the work hardening. From the results of uniaxial tension and compression, it was found that the mechanism that yielded a tensioncompression asymmetry was different between the RD and the TD: the difference in the twinning activity affected notably the asymmetry in the RD, whereas the difference in the slip activity played a significant role in the TD.

4. During TC loading, the following in-plane anisotropy was depicted: the work hardening increased slightly in the latter stage of compression in the $\mathrm{RD}$, whereas it remained almost unchanged in the TD. The reason for this anisotropy was that the deformation modes with high CRSS, such as pyramidal $\langle a+c\rangle$ slip and basal slip, were pronounced only in the latter stage of compression in the $\mathrm{RD}$, whereas the activities of basal slip and pyramidal $<a>$ slip were dominant throughout compression in the TD.

5. During CT loading, the following in-plane anisotropy was exhibited: the work-hardening rate changed rapidly, and a small but sharp stress peak appeared after the stress reversal in the RD. This tendency was pronounced for small compressive pre-strains. In contrast, in the TD, the work-hardening rate did not 
change rapidly after the stress reversal. The mechanism that yielded this difference between the RD and TD is as follows. After the stress reversal, the deformation modes changed rapidly from $\{10 \overline{1} 2\}$ detwinning to $\{11 \overline{2} 2\}$ twinning and pyramidal $\langle a+c\rangle$ slip in the $\mathrm{RD}$, whereas in the $\mathrm{TD}$, the dominant active deformation modes did not change rapidly. This rapid change in the active deformation modes in the RD resulted in the rapid change in the work-hardening rate, and thus the stress peak appeared in the RD.

\section{Acknowledgments}

The authors appreciate the kind support from Mr. Sohei Uchida of TRI Osaka in performing the EBSD measurements. This work was partially supported by JSPS KAKENHI Grant number 26289271, the Amada Foundation, and the Photon and Quantum Basic Research Coordinated Development Program from the Ministry of Education, Culture, Sports, Science and Technology, Japan.

Appendix A Constitutive equation for a single crystal

The present study utilized the rate-sensitivity crystal plasticity model formulated in the literature [Pierce et al., 1983; Asaro and Needleman, 1985]. The velocity gradient tensor $\boldsymbol{L}$ is assumed to be decomposed into an elastic part, $\boldsymbol{L}^{\mathrm{e}}$, and a plastic part, $\boldsymbol{L}^{\mathrm{p}}$, in the form

$$
\boldsymbol{L}=\boldsymbol{L}^{\mathrm{e}}+\boldsymbol{L}^{\mathrm{p}} .
$$

Assuming that the plastic velocity gradient $\boldsymbol{L}^{\mathrm{p}}$ results from the shear strain rate $\dot{\gamma}^{(\alpha)}$ due to slip or twinning activity yields

$$
\boldsymbol{L}^{\mathrm{p}}=\sum_{\alpha=1}^{N} \dot{\gamma}^{(\alpha)}\left(\boldsymbol{s}^{(\alpha)} \otimes \boldsymbol{m}^{(\alpha)}\right) \text {. }
$$

The plastic strain rate $\boldsymbol{D}^{\mathrm{p}}$ and the plastic spin $\boldsymbol{W}^{\mathrm{p}}$ are obtained by decomposing the plastic velocity gradient as follows

$$
\boldsymbol{D}^{\mathrm{p}}=\sum_{\alpha=1}^{N} \dot{\gamma}^{(\alpha)} \boldsymbol{p}^{(\alpha)}, \quad \boldsymbol{p}^{(\alpha)}=\frac{1}{2}\left(\boldsymbol{s}^{(\alpha)} \otimes \boldsymbol{m}^{(\alpha)}+\boldsymbol{m}^{(\alpha)} \otimes \boldsymbol{s}^{(\alpha)}\right),
$$

and

$$
\boldsymbol{W}^{\mathrm{p}}=\sum_{\alpha=1}^{N} \dot{\gamma}^{(\alpha)} \boldsymbol{\omega}^{(\alpha)}, \quad \boldsymbol{\omega}^{(\alpha)}=\frac{1}{2}\left(\boldsymbol{s}^{(\alpha)} \otimes \boldsymbol{m}^{(\alpha)}-\boldsymbol{m}^{(\alpha)} \otimes \boldsymbol{s}^{(\alpha)}\right) .
$$

The corotational stress rate with respect to the lattice spin, $\stackrel{\circ}{\sigma}^{\mathrm{e}}$, is assumed to be determined by the elastic strain rate, $\boldsymbol{D}^{\mathrm{e}}$, in the form 


$$
\stackrel{\circ}{\boldsymbol{\sigma}}^{\mathrm{e}}=\dot{\boldsymbol{\sigma}}-\boldsymbol{W}^{\mathrm{e}} \cdot \boldsymbol{\sigma}+\boldsymbol{\sigma} \cdot \boldsymbol{W}^{\mathrm{e}}=\boldsymbol{\sigma}+\boldsymbol{W}^{\mathrm{p}} \cdot \boldsymbol{\sigma}-\boldsymbol{\sigma} \cdot \boldsymbol{W}^{\mathrm{p}}=\boldsymbol{C}: \boldsymbol{D}^{\mathrm{e}},
$$

where $C$ is the elastic constitutive moduli. From eq. (8) and the second and third equations of eq. (10), the constitutive equation for a single crystal is eventually obtained as

$$
\stackrel{\circ}{\boldsymbol{\sigma}}=\boldsymbol{C}: \boldsymbol{D}-\sum_{\alpha=1}^{N} \dot{\gamma}^{(\alpha)}\left(\boldsymbol{C}: \boldsymbol{p}^{(\alpha)}+\boldsymbol{\omega}^{(\alpha)} \cdot \boldsymbol{\sigma}-\boldsymbol{\sigma} \cdot \boldsymbol{\omega}^{(\alpha)}\right) .
$$

\section{Appendix B Simulation procedure of plastic contour}

Denoting the prescribed stress ratio as $\alpha(\leq 1)$, the plastic contour under pseudo-linear stress paths was simulated using the following steps [Hama and Takuda, 2012b]:

(i) Displacement increments in the $x$ direction are assigned to the plane $x=l$, while fixing the plane $y=l$ in the $y$ direction.

(ii) Small displacement increments in the $y$ direction are assigned to the plane $y=l$, while fixing the plane $x=l$ in the $x$ direction.

(iii) If the condition $\alpha \approx \sigma_{y} / \sigma_{x}$ is satisfied, go to step (i). Otherwise, repeat step (ii) until the condition is satisfied.

In cases of $\alpha>1, x$ and $y$ in the above steps are replaced each other. The validity of the procedure was confirmed in the literature [Hama and Takuda, 2012b].

\section{References}

S.R. Agnew, M.H. Yoo and C.N. Tome, 2001. Application of texture simulation to understanding mechanical behavior of $\mathrm{Mg}$ and solid solution alloys containing $\mathrm{Li}$ or Y. Acta Mater., 49, 4277-4289.

K.E.K. Amouzou, T. Richeton, A. Roth, M.A. Lebyodkin, T.A. Lebedkina, 2016. Micromechanical modeling of hardening mechanisms in commercially pure alpha-titanium in tensile condition. Int. J. Plast., 80, 222-240.

E.A. Anderson, D.C. Jilson, S.R. Dunbar, 1953. Deformation mechanisms in alpha titanium. J. Met., 5, 1191-1197.

R.J. Asaro, A. Needleman, 1985. Texture development and strain hardening in rate dependent polycrystals. Acta Metallurgica 33, 923gica 
M. Battaini, E.V. Pereloma and C.H.J. Davies, 2007. Orientation Effect on Mechanical Properties of Commercially Pure Titanium at Room Temperature. Metall. Mater. Trans. A, 38A, 276-285.

N. Benmhenni, S. Bouvier, R. Brenner, T. Chauveau, B. Bacroix, 2013. Micromechanical modelling of monotonic loading of CP $\alpha$-Ti : Correlation between macroscopic and microscopic behaviour. Mater. Sci. Eng. A, 573, 222-233.

O. Bouaziz, N. Guelton, 2001. Modeling of TWIP effect on work-hardening. Mater. Sci. Eng. A, 319, 246-249.

O. Bouaziz, S. Allain, C. Scott, 2008. Effect of grain and twin boundaries on the hardening mechanisms of twinning-induced plasticity steels. Scr. Mater., 58.6, 484-487.

S. Bouvier, N. Benmhenni, W. Tirry, F. Gregory, M.E. Nixon, O. Cazacu, L. Rabet, 2012. Hardening in relation with microstructure evolution of high purity $\alpha$-titanium deformed under monotonic and cyclic simple shear loadings at room temperature. Mater. Sci. Eng. A, 535, 12-21.

D. R. Chichili, K. T. Ramesh, K. J. Hemker. 1998. The high-strain-rate response of alpha-titanium: experiments, deformation mechanisms and modeling. Acta Mater., 46, 1025-1043.

J. W. Christian, S. Mahajan, 1995. Deformation twinning. Prog. Mater. Sci., 39, 1-157.

Y.B. Chun, S.H. Yu, S.L. Semiatin, S.K. Hwang, 2005. Effect of deformation twinning on microstructure and texture evolution during cold rolling of CP-titanium. Mater. Sci. Eng. A, 398, 209-219.

H. Conrad, Effect of interstitial solutes on the strength ductility of titanium. 1981. Prog. Mater. Sci., 26, 123-403.

D. Gloaguen, B. Girault, J. Fajoui, V. Klosek, M.-J. Moya, 2016. In situ lattice strains analysis in titanium during a uniaxial tensile test. Mater. Sci. Eng. A, 662, 395-403.

S. Graff, W. Brocks and D. Steglich, 2007. Yielding of magnesium: From single crystal to polycrystalline aggregates. Int. J. Plast., 23, 1957-1978.

J. Gong, A.J. Wilkinson, 2009. Anisotropy in the plastic flow properties of single-crystal $\alpha$ titanium determined from micro-cantilever beams. Acta Mater., 57, 5693-5705.

N. P. Gurao, R. Kapoor, and S. Suwas, 2011. Deformation behaviour of commercially pure titanium at extreme strain rates. Acta Mater., 59, 3431-3446.

T. Hama and H. Takuda, 2011. Crystal-plasticity finite-element analysis of inelastic behavior during unloading in a magnesium alloy sheet. Int. J. Plast. 27, 1072-1092.

T. Hama, T., and H. Takuda, 2012a. Crystal plasticity finite-element simulation of deformation behavior in a magnesium alloy sheet considering detwinning. Steel Res. Int., Special Edition, 1115-1118.

T. Hama and H. Takuda, 2012b. Crystal plasticity finite-element simulation of 
work-hardening behavior in a magnesium alloy sheet under biaxial tension. Comput. Mater. Sci., 51, 156-164.

T. Hama, N. Kitamura and H. Takuda, 2013. Effect of twinning and detwinning on inelastic behavior during unloading in a magnesium alloy sheet. Mater. Sci. Eng. A, 583, 232-241.

T. Hama, H. Nagao, A. Kobuki, H. Fujimoto, H. Takuda, 2014. Work-hardening and twinning behaviors in a commercially pure titanium sheet under various loading paths. Mater. Sci. Eng. A, 620, 390-398.

T. Hama, T. Mayama, and H. Takuda, 2015. Deformation behavior of a magnesium alloy sheet with random crystallographic orientations under various loading paths. Romanian J. Tech. Sci.-Appl. Mech., 60, 16 pages.

T. Hama, Y. Tanaka, M. Uratani, and H. Takuda, 2016. Deformation behavior upon two-step loading in a magnesium alloy sheet. Int. J. Plast., 82, 283-304.

T. Hayashi, 2001. Press formability of commercially pure titanium sheet. Titan, 49, 184-190.

X. Huang, K. Suzuki and Y. Chino, 2010. Improvement of stretch formability of pure titanium sheet by differential speed rolling. Scr. Mater., 63, 473-476.

M. Ishiki, T. Kuwabara, M. Yamaguchi, Y. Maeda, Y. Hayashida and Y. Itsumi, 2009. Differential work hardening behavior of pure titanium sheet under biaxial loading.Trans. Jpn. Soc. Mech. Eng., 75, 491-500.

M. Ishiki, T. Kuwabara, and Y. Hayashida, 2011. Measurement and analysis of differential work hardening behavior of pure titanium sheet using spline function. Int. J. Mater. Form., 4, 193-204.

S. Ishiyama, S. Hanada and O. Izumi, 1990. Orientation Dependence of Twinning in Commercially Pure Titanium. J. Jpn. Inst. Met., 54, 976-984.

S. Ishiyama, 2006. Plastic deformation and press formability of C.P. titanium sheet. Titan. Jpn., 54, 42-51.

K. Kowalczyk-Gajewska, K. Sztwiertnia, J. Kawalko, K. Wierzbanowski, M. Wronski, K. Frydrich, S. Stupkiewicz, and H. Petryk, 2015. Texture evolution in titanium on complex decormation paths: Experiment and modelling. Mater. Sci. Eng. A, 637, 251-263.

Y. Kuchinomachi, Springback characteristics of a commercially pure titanium sheet in draw bending, Master thesis of Kyoto Univesity, 2015.

D. Lee, W. A. Backofen, 1966. An experimental determination of the yield locus for titanium and titanium-alloy sheet. Trans. Metall. Soc. AIME, 236, 1077-1084.

A. Marchenko, M. Mazière, S. Forest, J.-L. Strudel, 2016. Crystal plasticity simulation of strain aging phenomena in $\alpha$-titanium at room temperature. Int. J. Plast., 85, 1-33. 
S. Mullins, B. M. Patchett, 1981. Deformation microstructures in titanium sheet metal. Metall. Trans. A, 12, 853-863.

H. Nasiri-Abarbekoh, A. Ekrami, A.A. Ziaei-Moayyed, M. Shohani, 2012. Effects of rolling reduction on mechanical properties anisotropy of commercially pure titanium. Mater. Des., 34, 268-274.

S. Nemat-Nasser, W.G. Guo and J.Y. Cheng, 1999. Mechanical properties and deformation mechanism of a commercially pure titanium. Acta Mater., 47, 3705-3720.

M. E. Nixon, O. Cazacu, R. A. Lebensohn, 2010. Anisotropic response of high-purity $\alpha$-titanium: Experimental characterization and constitutive modeling. Int. J. Plast., 26, 516-532.

H. Numakura, Y. Minonishi, M. Koiwa, 1986. $<\overline{1} \overline{1} 23>\{10 \overline{1} 1\}$ slip in titanium polycrystals at room temperature. Scr. Metall. 20, 1581-1586.

N.E. Paton, W.A. Backofen, 1970. Plastic deformation of Titanium at elevated Temperatures. Metall. Trans., 1, 2839-2847.

D. Pierce, C.F. Shi, A. Needleman, 1984. A tangent modulus method for rate dependent solids. Comput. Struct. 18-5, 875-887.

D. Pierce, R.J. Asaro, A. Needleman, 1983. Material rate dependence and localized deformation in crystalline solids. Acta Metallurgica 31, 1951ica 31

M. J. Philippe, M. Serghat, P. Van Houtte and C. Esling, 1995. Modelling of texture evolution for materials of hexagonal symmetry - II. Application to zirconium and titanium or near alloys. Acta Metall. Mater., 43, 1619-1630.

F. D. Rosi, C.A. Dube, and B. H. Alexander, 1953. Mechanism of plastic flow in titanium -determination of slip and twinning elements. J. Met., 5, 257-265.

A. Roth, M. A. Lebyodkin, T. A. Lebedkina, J. -S. Lecomte, T. Richeton, K. E. K. Amouzou, 2014. Mechanisms of anisotropy of mechanical properties of $\alpha$-titanium in tension conditions. Mater. Sci. Eng. A, 596, 236-243.

S. Sinha, A. Ghosh, and N.P. Grao, 2016. Effect of initial orientation on the tensile properties of commercially pure titanium. Philos. Mag., 96, 1485-1508.

W. Tirry, S. Bouvier, N. Benmhenni, W. Hammami, A. M. Habraken, F. Coghe, D. Schryvers, L. Rabet, 2012. Twinning in pure Ti subjected to monotonic simple shear deformation. Mater. Charact., 72, 24-36.

C.N. Tome, R.A. Lebensohn, U.F. Kocks, 1991. A model for texture development dominated by deformation twinning: Application to zirconium alloys. Acta Metall. Mater., 39, 2667-2680.

P. Van Houtte, 1978. Simulation of the rolling and shear texture of brass by the Taylor theory adapted for mechanical twinning. Acta Metall., 26, 591-604. 
L. Wang, R.I. Barabash, Y. Yang, T.R. Bieler, M.A.crimp, P. Eisenlohr, W. Liu, G.E. Ice, 2011. Experimental characterization and crystal plasticity modeling of heterogeneous deformation in polycrystalline $\alpha$-Ti. Metall. Mater. Trans. A, 42, 626-635.

H. Wang, P.D. Wu, J. Wang, C.N. Tome, 2013. A crystal plasticity model for hexagonal close packed (HCP) crystals including twinning and de-twinning mechanisms. Int. J. Plast. 49, 36-52.

J. L. W. Warwick, N. G. Jones, K. M. Rahman, D. Dye, 2012. Lattice strain evolution during tensile and compressive loading of CP Ti. Acta Mater., 60, 6720-6731.

X. Wu, S. R. Kalidindi, C. Necker, A. A. Salem, 2007. Prediction of crystallographic texture evolution and anisotropic stress-strain curves during large plastic strains in high purity $\alpha$-titanium using a Taylor-type crystal plasticity model. Acta Mater., 55, 423-432.

Y. Yamada, N. Yoshimura, and T. Sakurai, 1968. Plastic stress-strain matrix and its application for the solution of elastic-plastic problems by the finite element method. Int. J. Mech. Sci., 10, 343-354.

Y. Yang, L. Wang, T. R. Bieler, P. Eisenlohr, and M. A. Crimp, 2011. Quantitative atomic force microscopy characterization and crystal plasticity finite element modeling of heterogeneous deformation in commercial purity titanium. Metall. Mater. Trans. A, 42A, 636-644.

N. Yi, T. Hama, A. Kobuki, H. Fujimoto, H. Takuda, 2016. Anisotropic deformation behavior under various strain paths in commercially pure titanium Grade 1 and Grade 2 sheets. Mater. Sci. Eng. A, 655, 70-85.

E. Yu, I. Kim, D.H. Shin and J. Kim, 2008. Deformation Mechanism of Severely Deformed CP-Titanium by Uniaxial Compression Test. Mater. Trans., 49, 38-40.

C. Zambaldi, Y. Yang and T. R. Bieler, 2012. Orientation informed nanoindentation of $\alpha$-titanium: Indentation pileup in hexagonal metals deforming by prismatic slip. J. Mater. Res., 27, 356-367.

Z. Zeng, Y. Zhang, S. Jonsson, 2009. Deformation behavior of commercially pure titanium during simple hot compression. Mater. Des., 30, 3105-3111.

\section{List of Figure and Table captions}

Table 1 Tensile properties of the CP-Ti sheet in RD and TD [Yi et al., 2016].

Table 2 Latent-hardening parameters.

Table 3 Determined hardening parameters for each slip and twinning system, in MPa. 
Fig. 1 Finite-element model used in the present simulation.

Fig. 2 Initial pole figures. (a) Experimental results obtained using EBSD measurement [Yi et al., 2016], and results obtained from crystallographic orientations used in simulations for finite-element models with (b) 343 elements and (c) 125 elements.

Fig. 3 Stress-strain curves under uniaxial tension obtained with five different sets of initial crystallographic orientations for models with (a) $125(=5 \times 5 \times 5)$ elements and (b) $343(=7 \times 7 \times 7)$ elements.

Fig. 4 Absolute stress-absolute strain curves obtained from experiment (dotted lines) [Yi et al., 2016] and simulation (solid lines) under tension and compression.

Fig. 5 Evolution of work-hardening rate under tension and compression in RD and TD obtained from (a) experiment [Yi et al., 2016] and (b) simulation.

Fig. 6 Evolution of Lankford value obtained under tension in RD and TD.

Fig. 7 Stress-strain curves under TC loading obtained from experiment (dotted lines) [Yi et al., 2016] and simulation (solid lines) in (a) RD and (b) TD.

Fig. 8 Variations of work-hardening rate after stress reversal under TC loading in RD obtained from (a) experiment [Yi et al., 2016] and (b) simulation.

Fig. 9 Variations of work-hardening rate after stress reversal under TC loading in TD obtained from (a) experiment and (b) simulation.

Fig. 10 (0001) and (1010) pole figures under TC loading in RD obtained from experiment [Yi et al., 2016] and simulation. Results are at points (a) A and (b) B shown in Fig. 7(a).

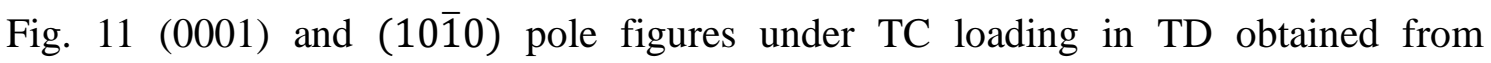
experiment and simulation. Results are at points (a) C and (b) D shown in Fig. 7(b).

Fig. 12 Stress-strain curves under CT loading in (a) RD and (b) TD obtained from experiment (dotted lines) [Yi et al., 2016] and simulation (solid lines).

Fig. 13 Variations of work-hardening rate after stress reversal under CT loading in RD obtained from (a) experiment [Yi et al., 2016] and (b) simulation.

Fig. 14 Variations of work-hardening rate after stress reversal under CT loading in TD obtained from (a) experiment [Yi et al., 2016] and (b) simulation.

Fig. 15 Relationship between $g_{T}$ and compressive pre-strain obtained from experiment and simulation.

Fig. 16 (0001) and (1010) pole figures obtained under CT loading in RD obtained from experiment [Yi et al., 2016] and simulation. Results are at points (a) E, (b) F, (c) G, and (d) H shown in Fig. 12(a).

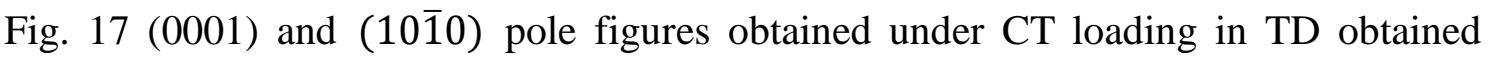


from experiment and simulation. Results are at points (a) I and (b) J shown in Fig. 12(b).

Fig. 18 Normalized contours of plastic work obtained from simulation.

Fig. 19 Variations of shape ratio of stress points $\left(L / L_{0}\right)$ in each loading direction as a function of plastic strain.

Fig. 20 Evolution of relative activity during tension in (a) RD and (b) TD.

Fig. 21 Absolute stress-absolute strain curves under tension and compression obtained with (dotted lines) and without (solid lines) considering twinning activity.

Fig. 22 Evolution of work-hardening rates under tension and compression obtained with (dotted lines) and without (solid lines) considering twinning activity.

Fig. 23 Evolution of relative activities as a function of plastic work for biaxial stress ratios $\sigma_{x}: \sigma_{y}=$ (a) $1: 0$, (b) $4: 1$, (c) $2: 1$, (d) $4: 3$, and (e) $1: 1$.

Fig. 24 Evolution of relative activities as a function of plastic work for biaxial stress ratios $\sigma_{x}: \sigma_{y}=$ (a) 3:4, (b) $1: 2$, (c) $1: 4$, and (d) $0: 1$.

Fig. 25 Relationship between relative activity and biaxial stress ratio at plastic strain of 0.001 .

Fig. 26 Evolution of relative activities obtained under compression in (a) RD and (b) TD.

Fig. 27 Evolution of relative activities obtained under TC loading in the case of tensile pre-strain of 0.1 in (a) RD and (b) TD.

Fig. 28 Evolution of relative activities obtained under CT loading in RD in the case of compressive pre-strain of (a) 0.03 and (b) 0.1 .

Fig. 29 Evolution of relative activities obtained under CT loading in TD in the case of compressive pre-strain of (a) 0.05 and (b) 0.1 . 


\section{Figures and Tables}

Table 1 Tensile properties of the CP-Ti sheet in RD and TD [Yi et al., 2016].

\begin{tabular}{ccccc}
\hline & $\boldsymbol{\sigma}_{\mathbf{T}} / \mathrm{MPa}$ & $\boldsymbol{\sigma}_{\mathbf{0 . 2}} / \mathrm{MPa}$ & $F / \mathrm{MPa}$ & $n$ \\
\hline $\mathrm{RD}$ & 303 & 153 & 500 & 0.213 \\
$\mathrm{TD}$ & 273 & 203 & 424 & 0.143 \\
\hline
\end{tabular}

$\sigma_{\mathrm{T}}$ denotes the ultimate tensile strength, and $\sigma_{0.2}$ denotes the $0.2 \%$ proof stress. The true stress-true strain curve was approximated using the equation $\sigma=F \varepsilon^{n}$. 


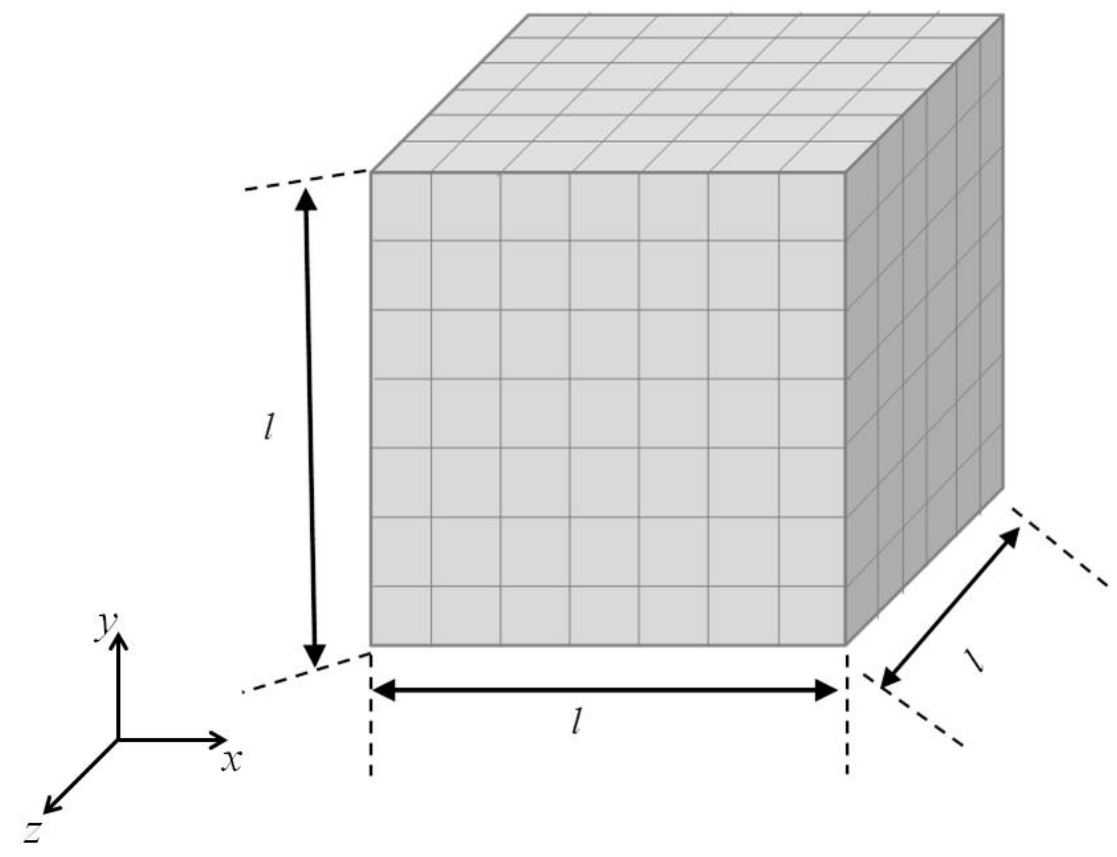

Fig. 1 Finite-element model used in the present simulation. 
(a)
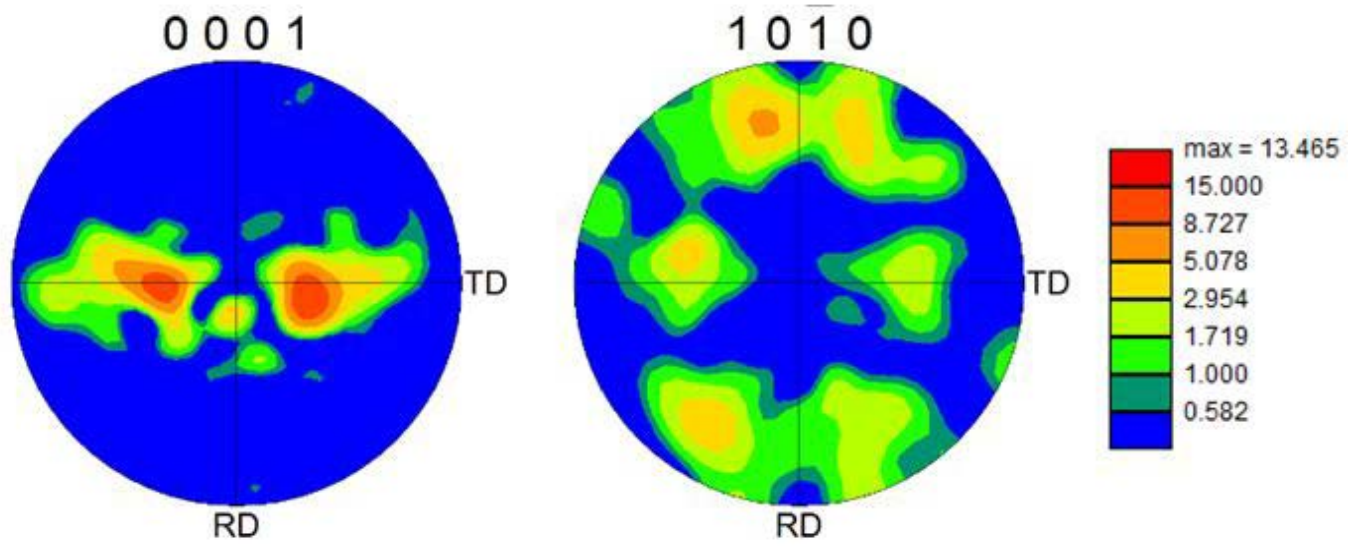

(b)

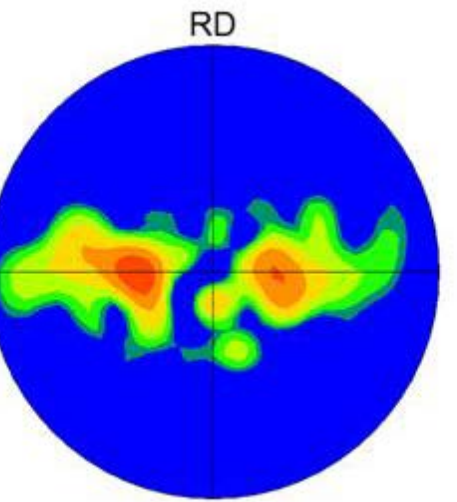

(c)
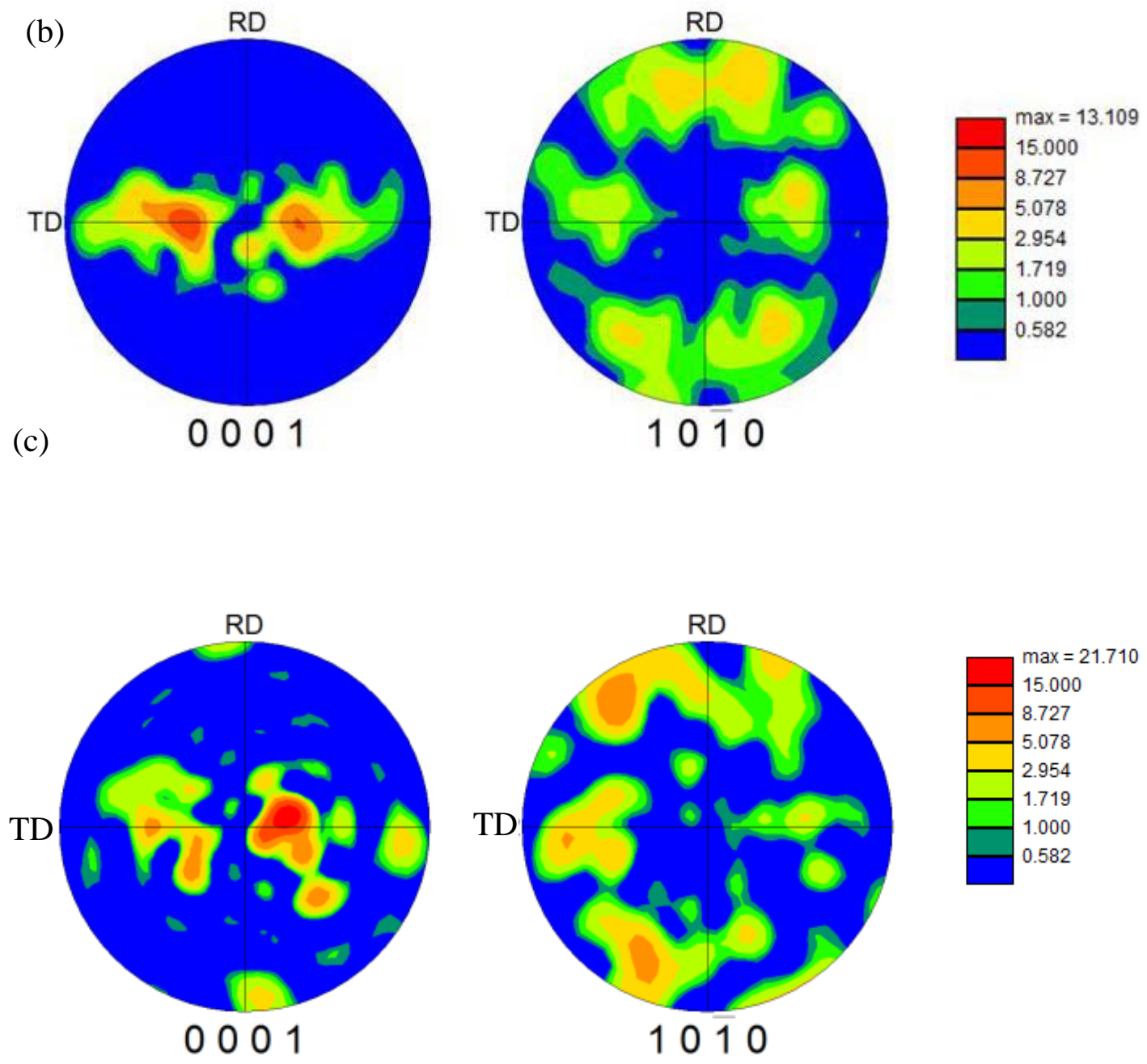

Fig. 2 Initial pole figures. (a) Experimental results obtained using EBSD measurement [Yi et al., 2016], and results obtained from crystallographic orientations used in simulations for finite-element models with (b) 343 elements and (c) 125 elements. 
Table 2 Latent-hardening parameters.

\begin{tabular}{llllllll}
\hline & Basal $<a>$ & Prism $<a>$ & Pyr $<a>$ & Pyr $<a+c>-1$ & Pyr $<a+c>-2$ & $\{10 \overline{1} 2\}$ twin & $\{11 \overline{2} 2\}$ twin \\
\hline Basal $<a>$ & 0.5 & 0.5 & 0.5 & 0.5 & 0.5 & 0.9 & 0.9 \\
Prism $<a>$ & 0.5 & 0.5 & 0.5 & 0.5 & 0.5 & 0.9 & 0.9 \\
Pyr $<a>$ & 0.5 & 0.5 & 0.5 & 0.5 & 0.5 & 0.9 & 0.9 \\
Pyr $<a+c>-1$ & 0.5 & 0.5 & 0.5 & 0.5 & 0.5 & 0.9 & 2.0 \\
Pyr $<a+c>-2$ & 0.5 & 0.5 & 0.5 & 0.5 & 0.5 & 0.9 & 2.0 \\
$\{10 \overline{1} 2\}$ twin & 0.5 & 0.5 & 0.5 & 0.5 & 0.5 & 0.9 & 0.9 \\
$\{11 \overline{2} 2\}$ twin & 0.5 & 0.5 & 0.5 & 0.5 & 0.5 & 0.9 & 0.9 \\
\hline
\end{tabular}


Table 3 Determined hardening parameters for each slip and twinning system, in MPa.

\begin{tabular}{cccccccc}
\hline & Basal & Prism & Pyr $<a>$ & Pyr $<a+c>-1$ & Pyr $<a+c>-2$ & $\{\mathbf{1 0 1} 2\}$ & $\{\mathbf{1 1} \overline{2} 2\}$ \\
\hline $\boldsymbol{\tau}_{\mathbf{0}}$ & 133 & 62 & 81 & 145 & 145 & 90 & 140 \\
$\boldsymbol{\tau}_{\infty}$ & 180 & 160 & 210 & 270 & 270 & & \\
$\boldsymbol{h}_{\mathbf{0}}$ & 1950 & 1050 & 580 & 2050 & 2050 & 350 & 350 \\
\hline
\end{tabular}


(a)

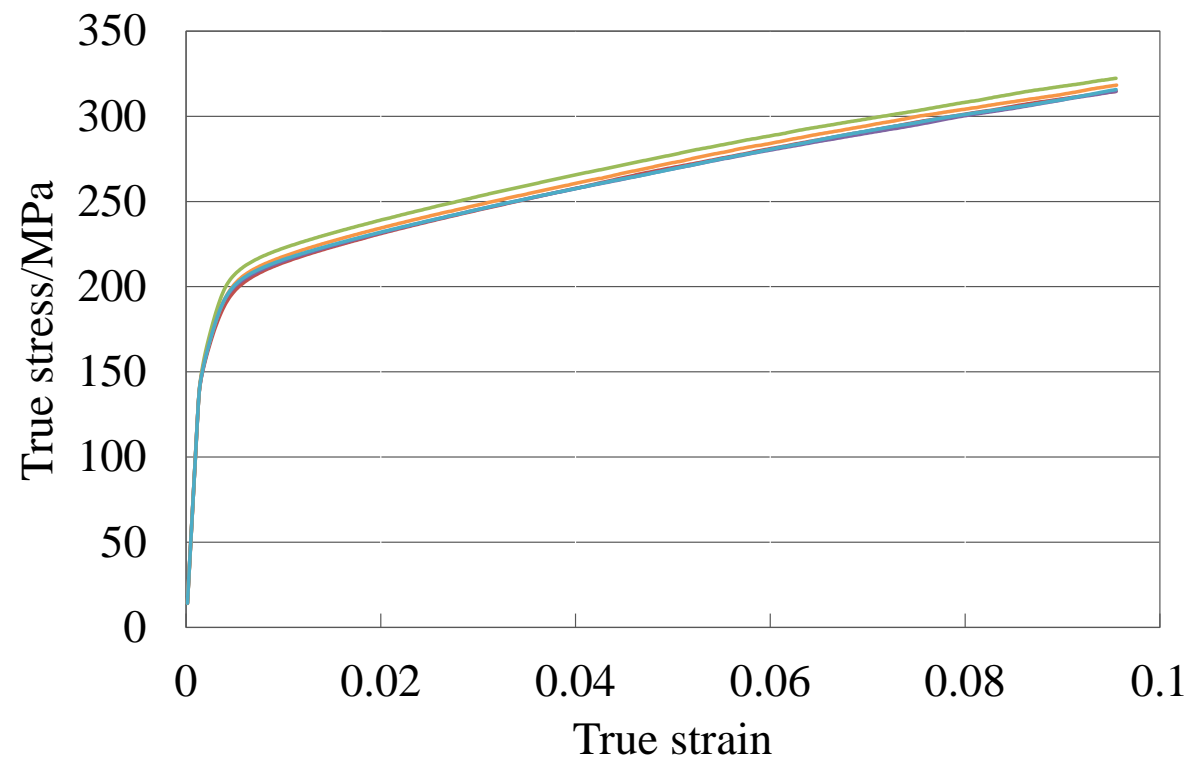

(b)

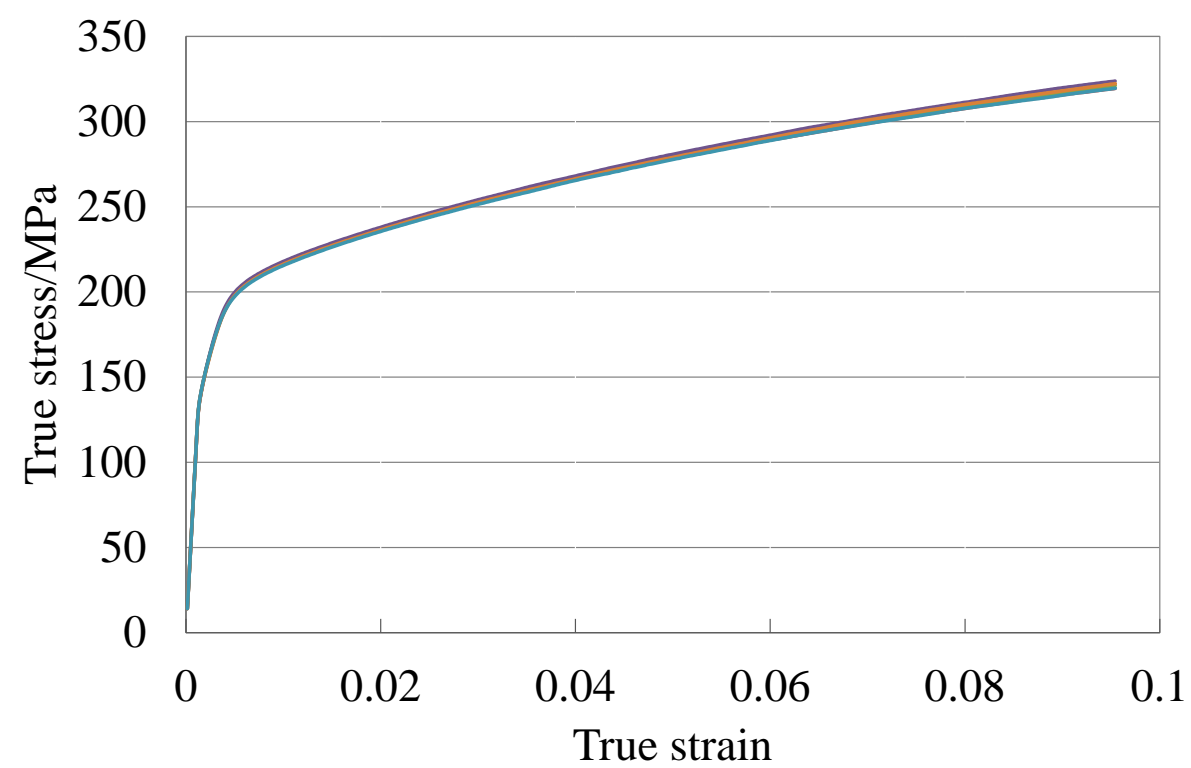

Fig. 3 Stress-strain curves under uniaxial tension obtained with five different sets of initial crystallographic orientations for models with (a) $125(=5 \times 5 \times 5)$ elements and (b) $343(=7 \times 7 \times 7)$ elements. 


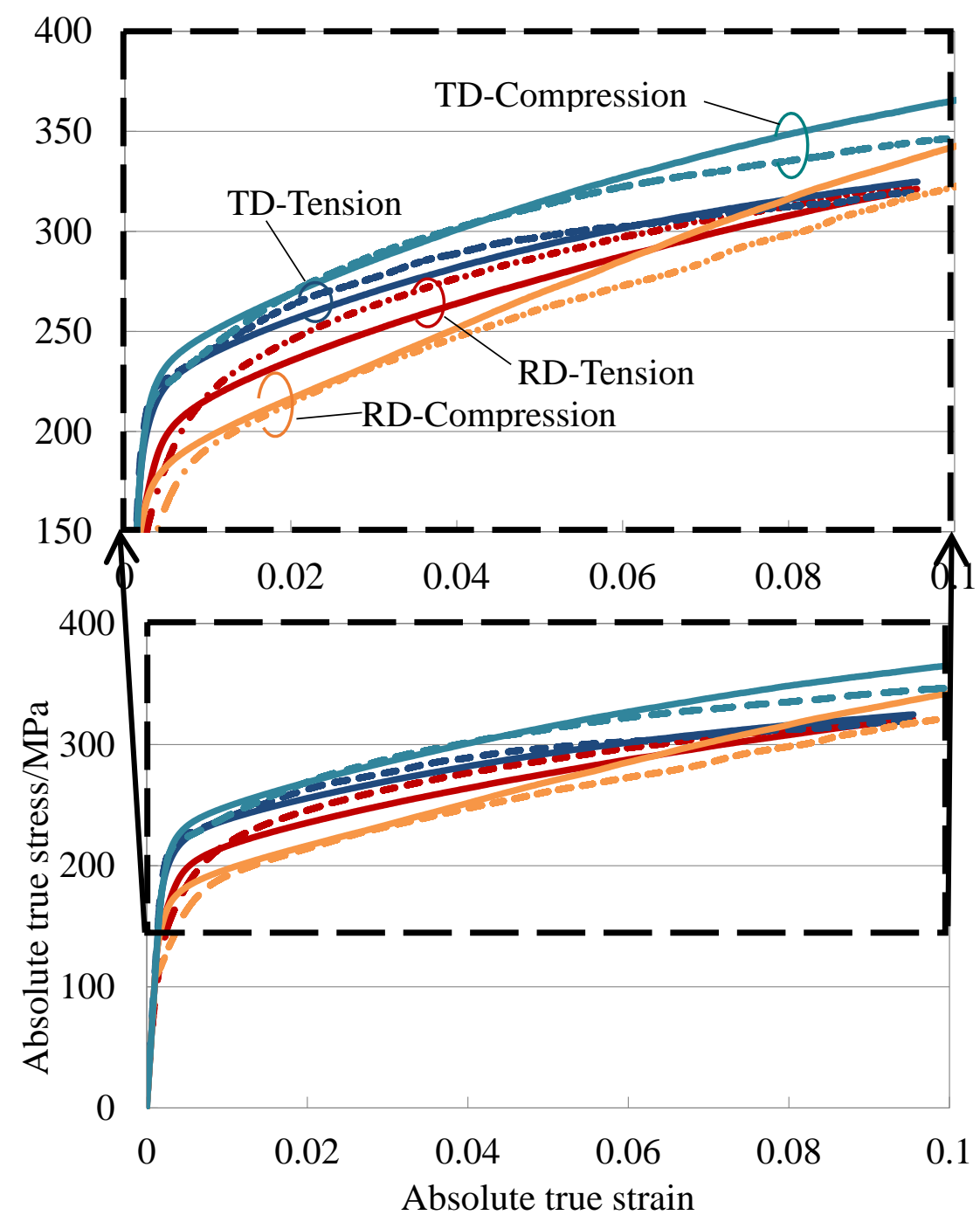

Fig. 4 Absolute stress-absolute strain curves obtained from experiment (dotted lines) [Yi et al., 2016] and simulation (solid lines) under tension and compression. 
(a)

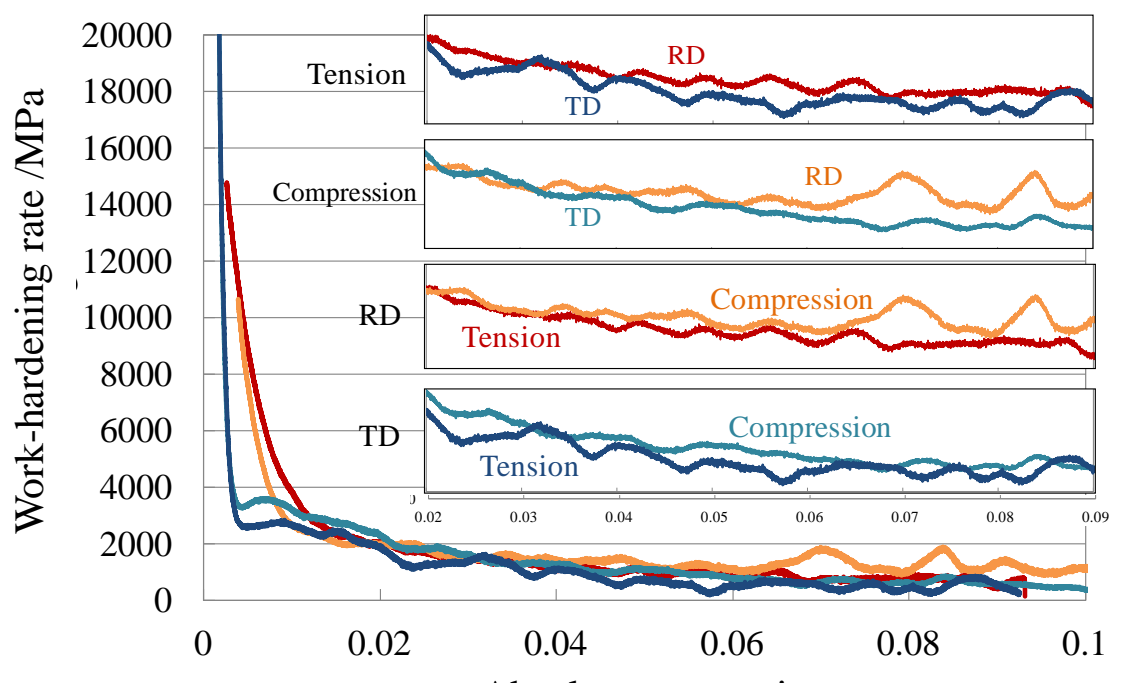

Absolute true strain

(b)

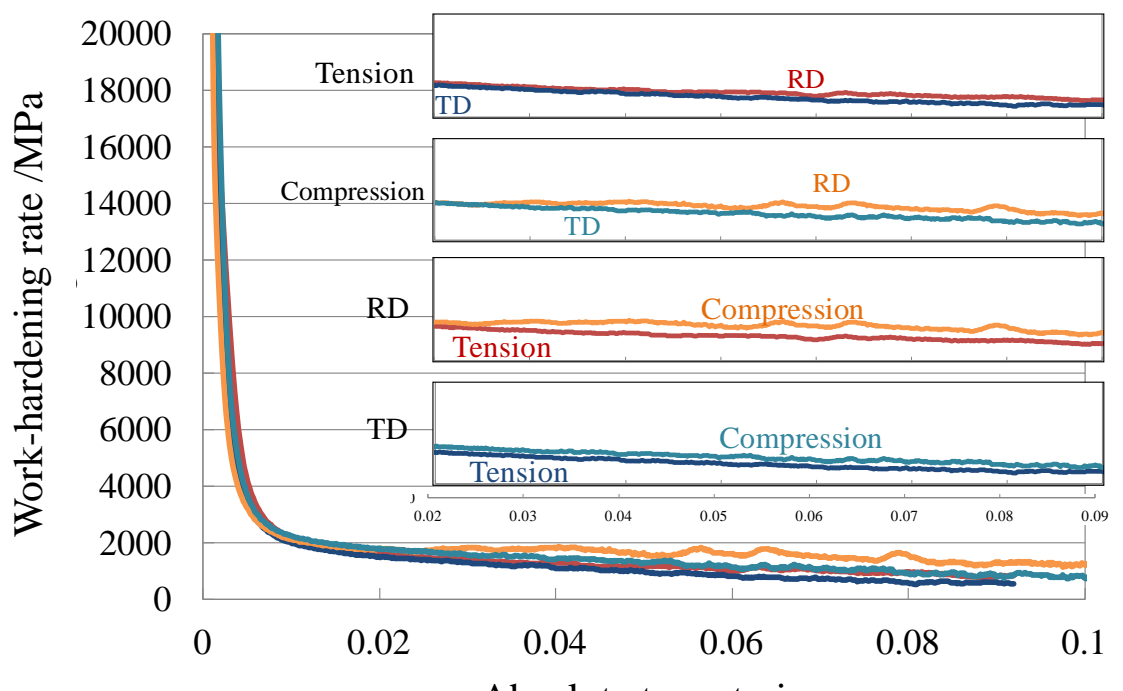

Absolute true strain

Fig. 5 Evolution of work-hardening rate under tension and compression in RD and TD obtained from (a) experiment [Yi et al., 2016] and (b) simulation. 


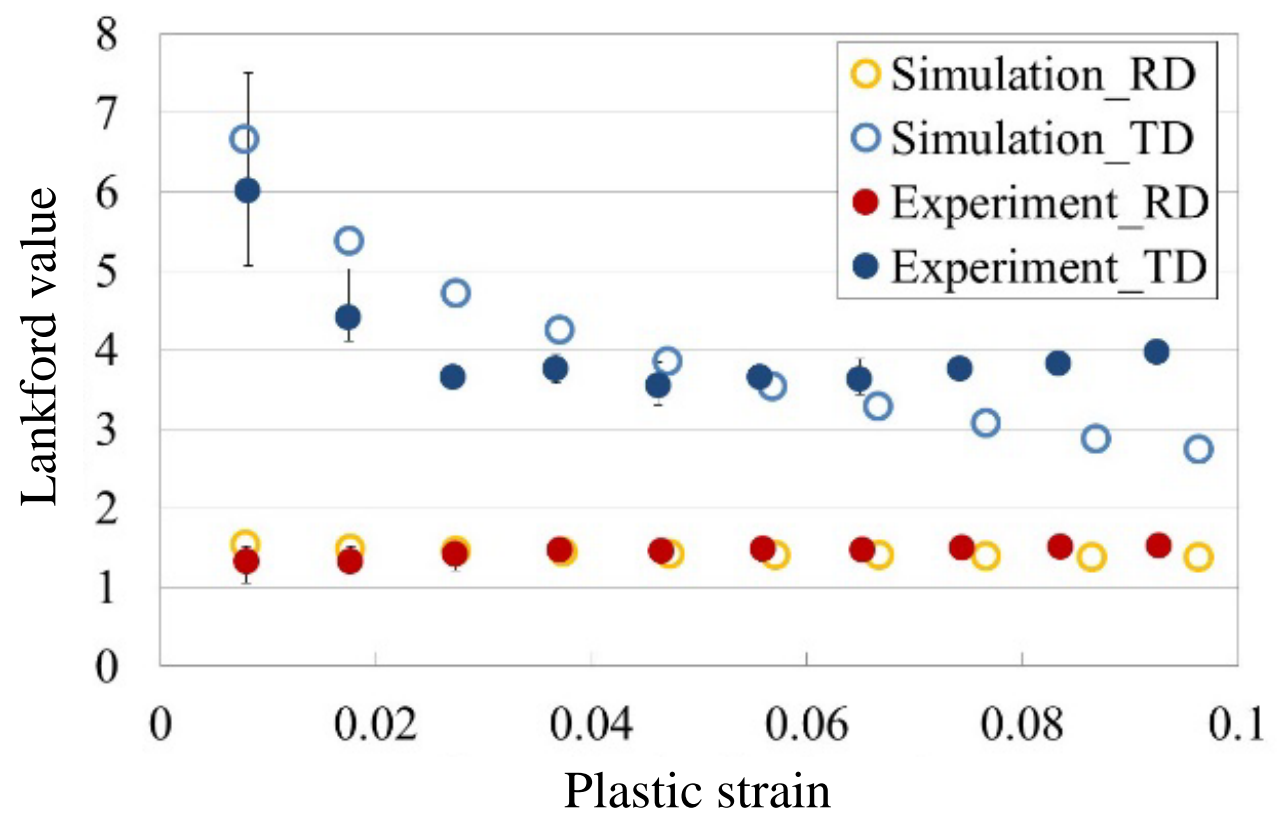

Fig. 6 Evolution of Lankford value obtained under tension in RD and TD. 
(a)

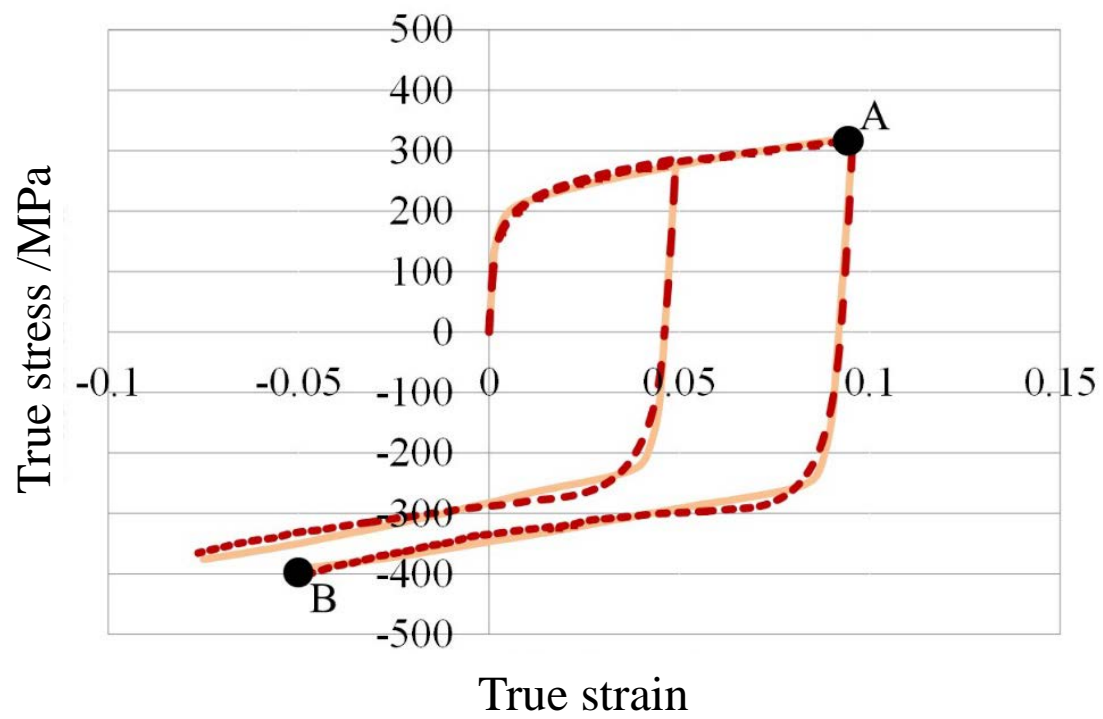

(b)

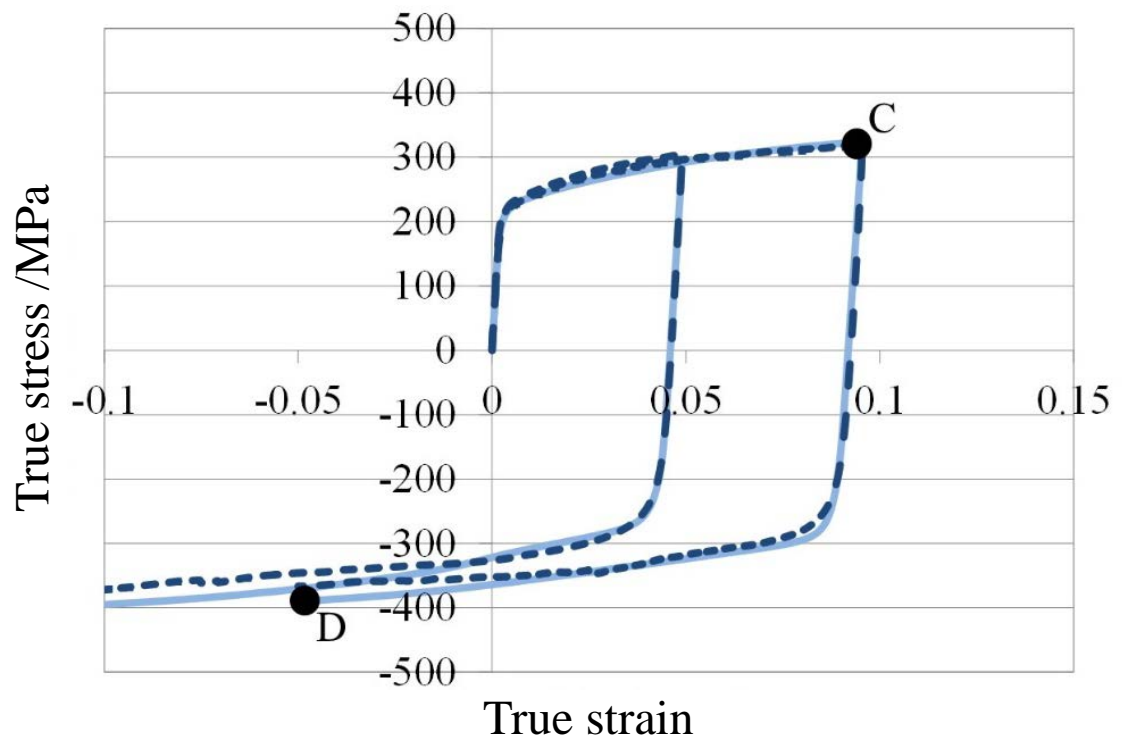

Fig. 7 Stress-strain curves under TC loading obtained from experiment (dotted lines) [Yi et al., 2016] and simulation (solid lines) in (a) RD and (b) TD. 
(a)

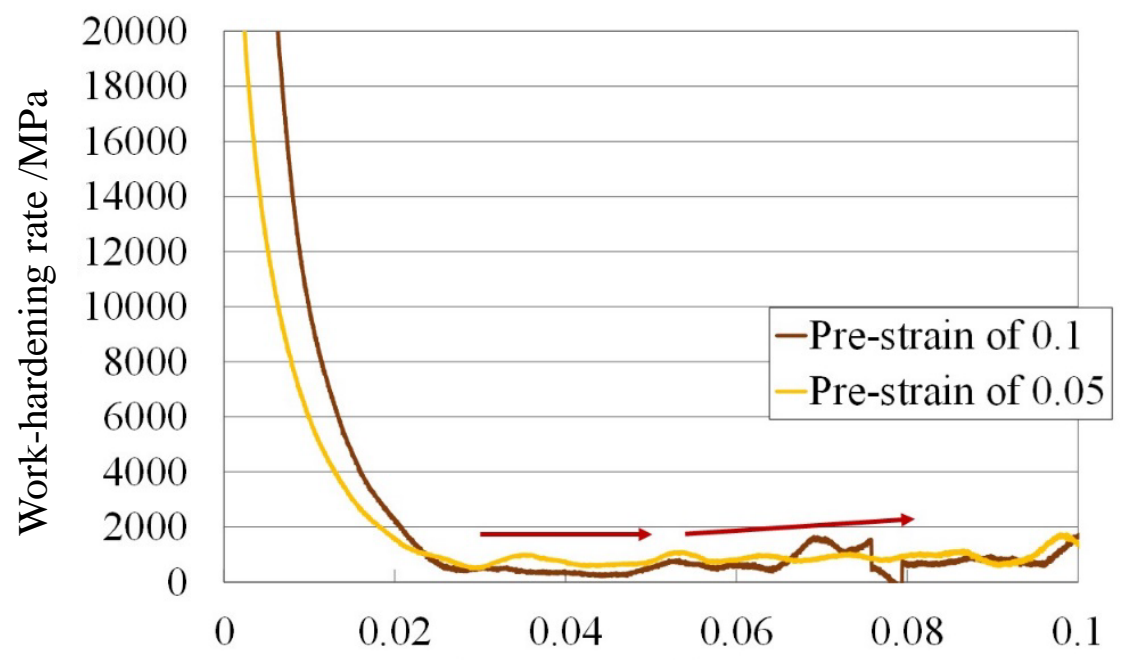

Absolute true strain

(b)

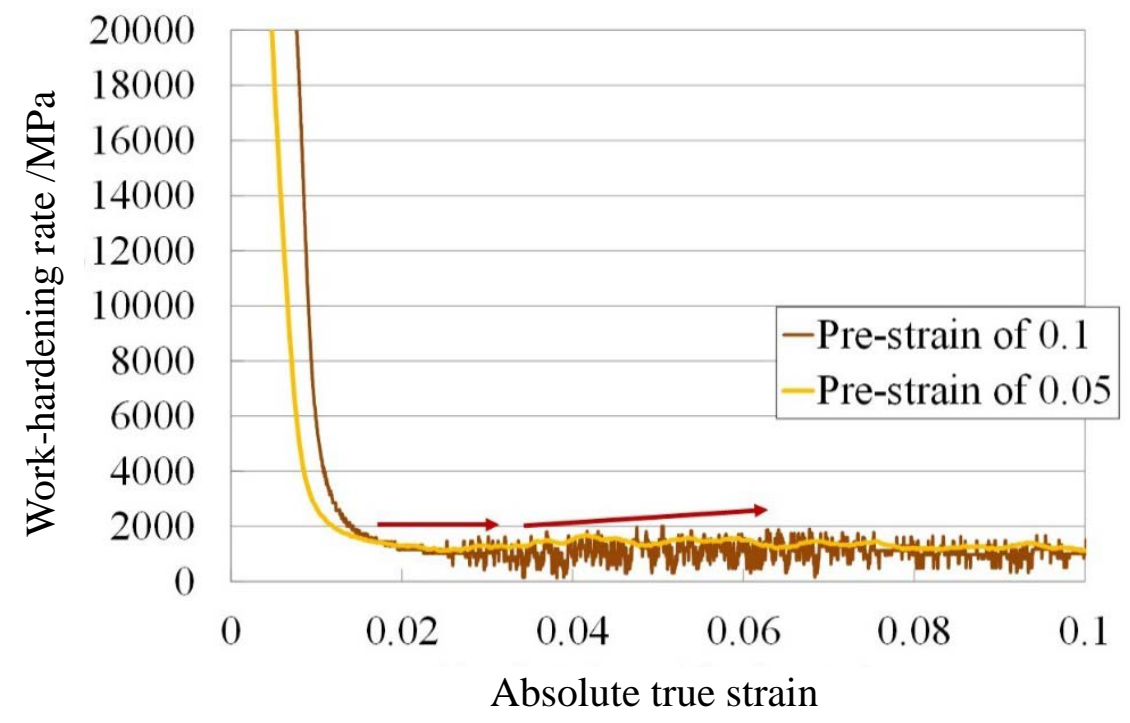

Fig. 8 Variations of work-hardening rate after stress reversal under TC loading in RD obtained from (a) experiment [Yi et al., 2016] and (b) simulation. 
(a)

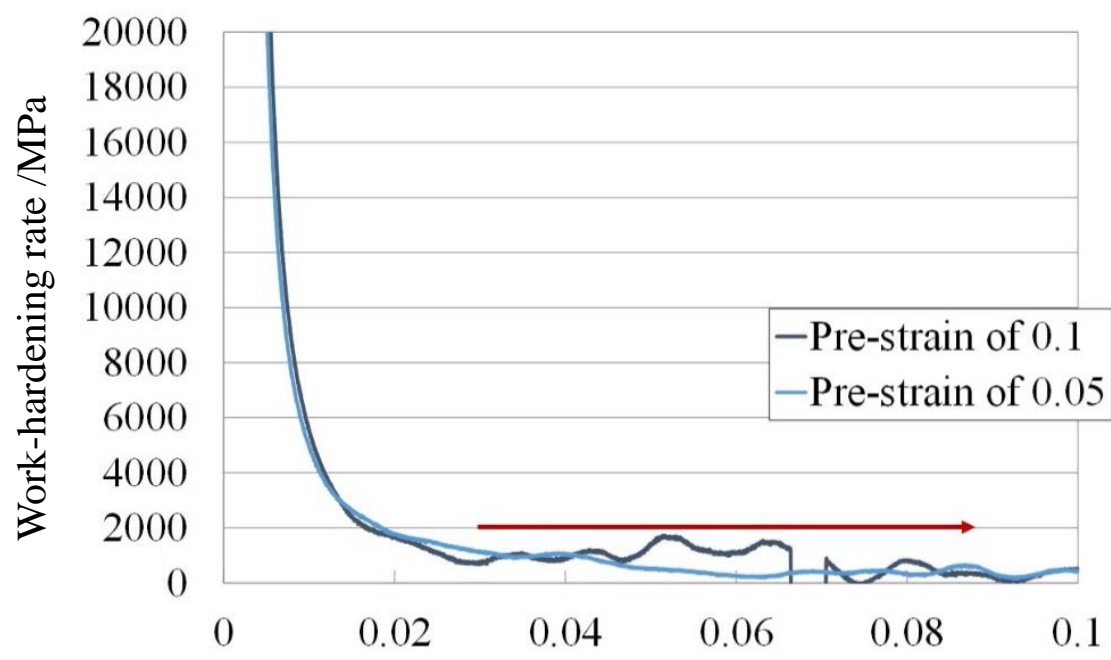

Absolute true strain

(b)

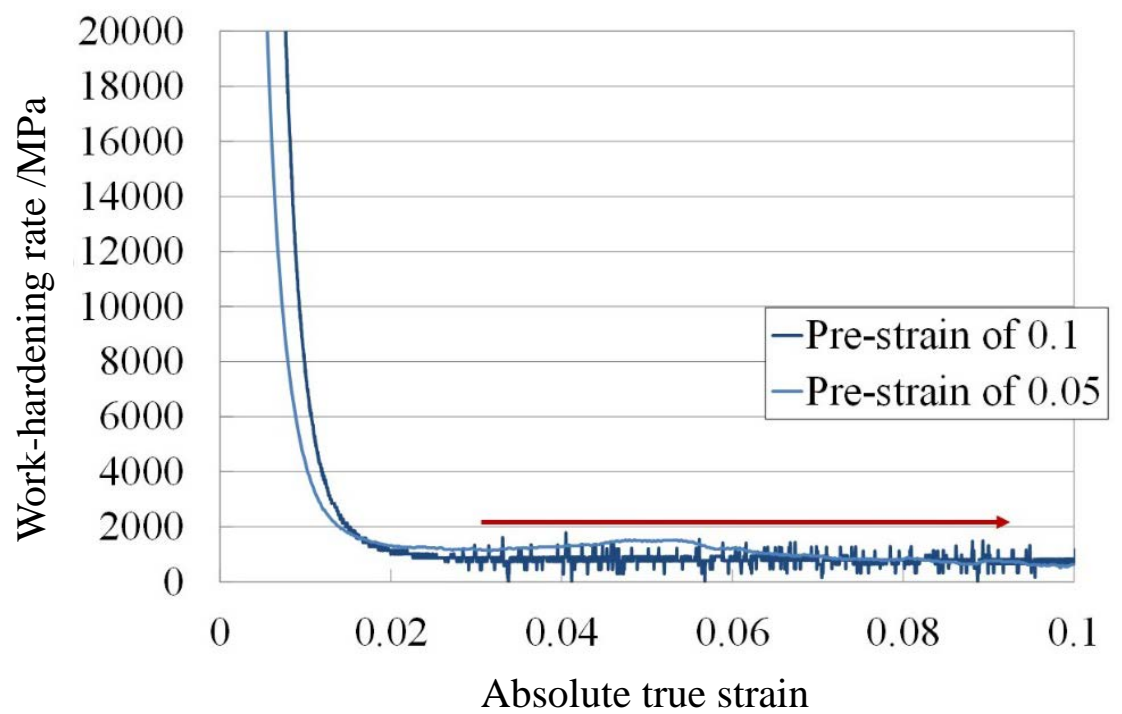

Fig. 9 Variations of work-hardening rate after stress reversal under TC loading in TD obtained from (a) experiment and (b) simulation. 
(a)

\section{Experiment}
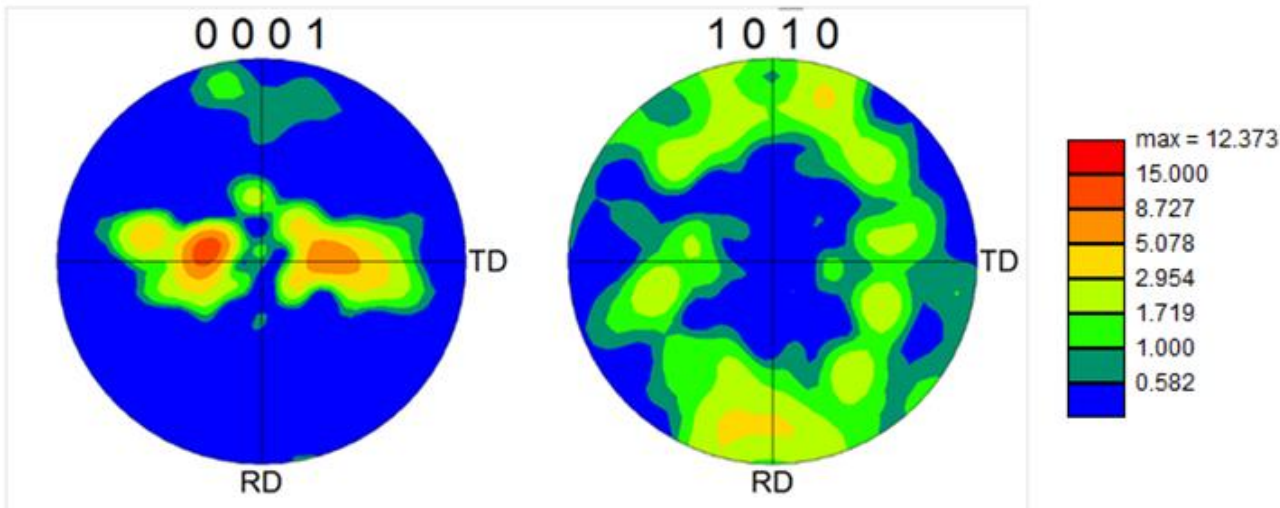

Simulation
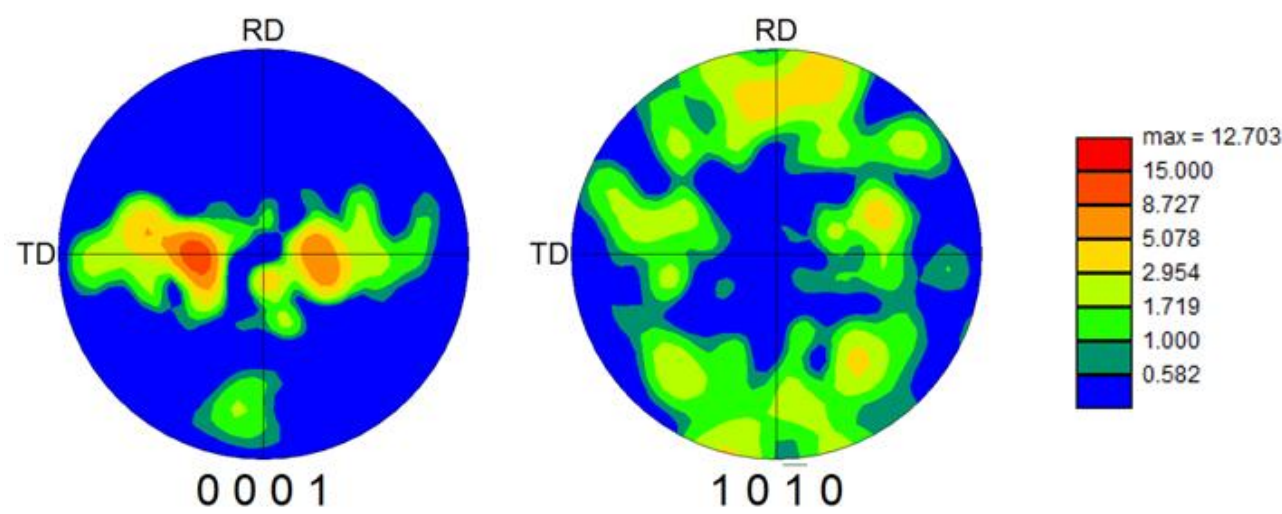
(b)

\section{Experiment}
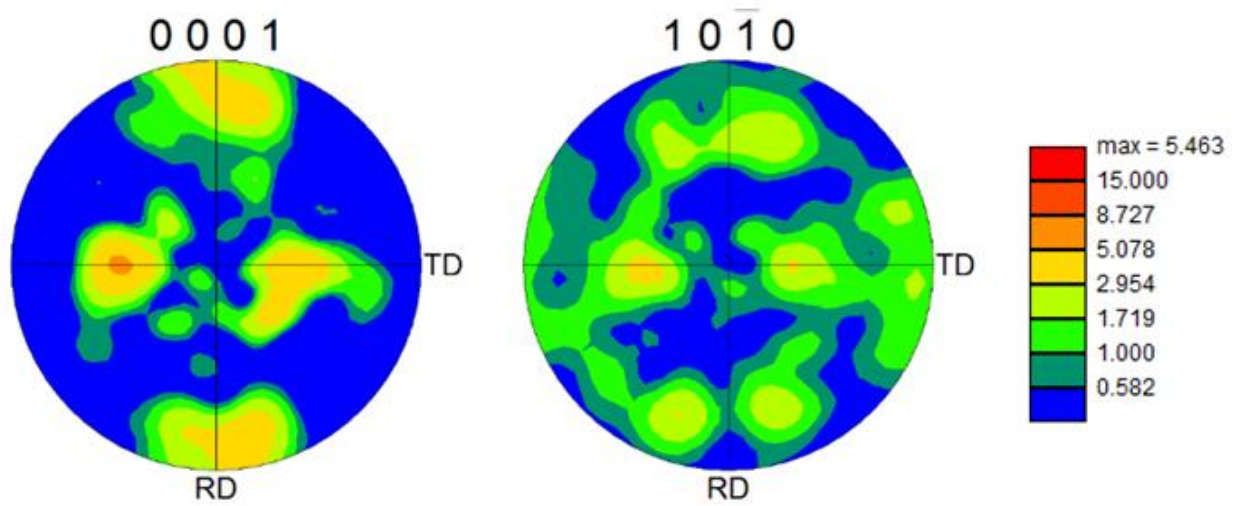

Simulation
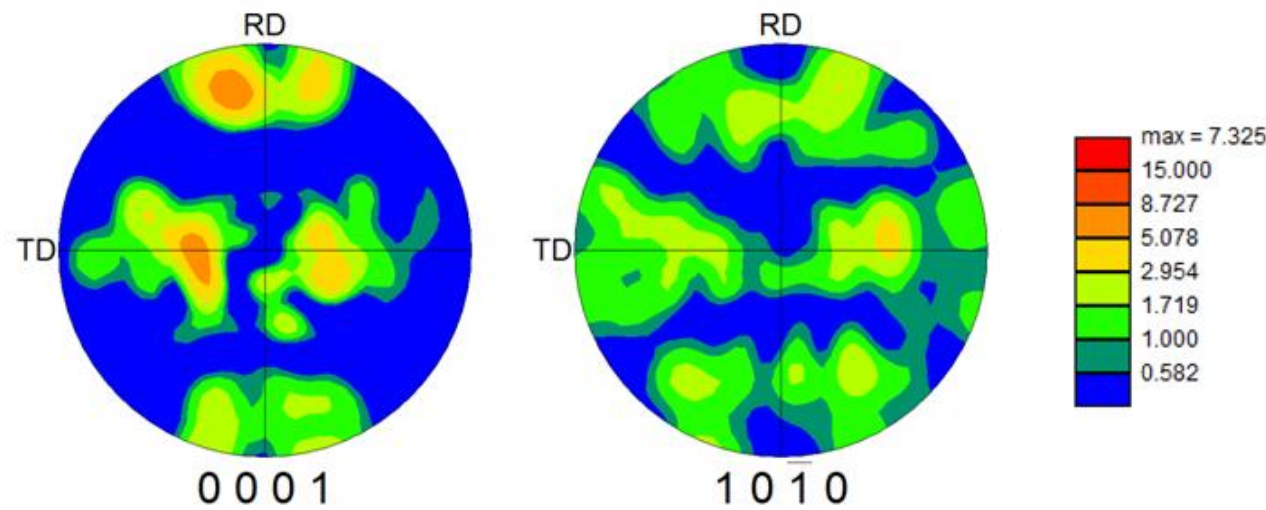

Fig. 10 (0001) and (1010) pole figures under TC loading in RD obtained from experiment [Yi et al., 2016] and simulation. Results are at points (a) A and (b) B shown in Fig. 7(a). 
(a)

\section{Experiment}
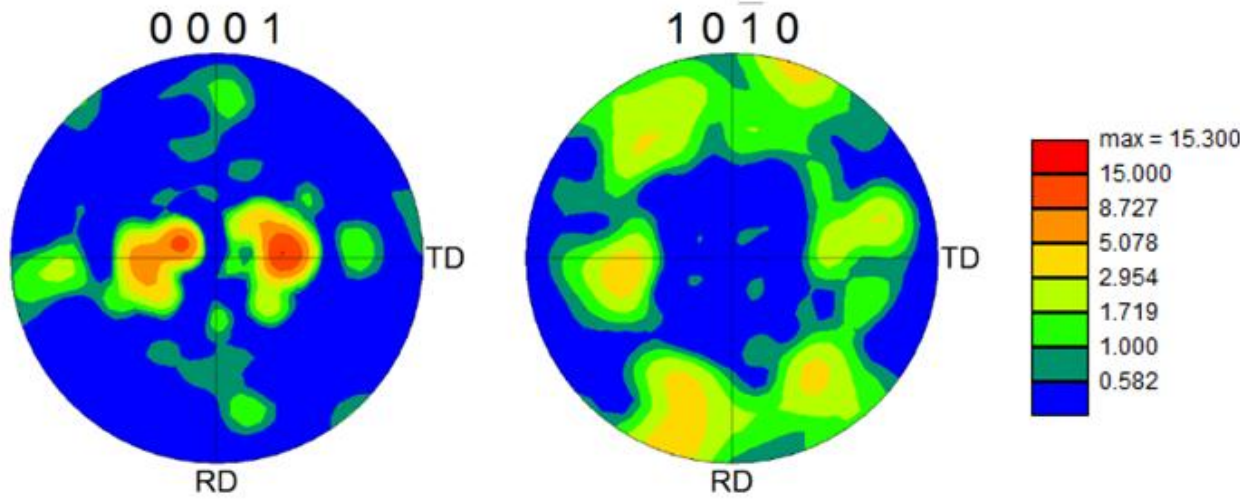

Simulation
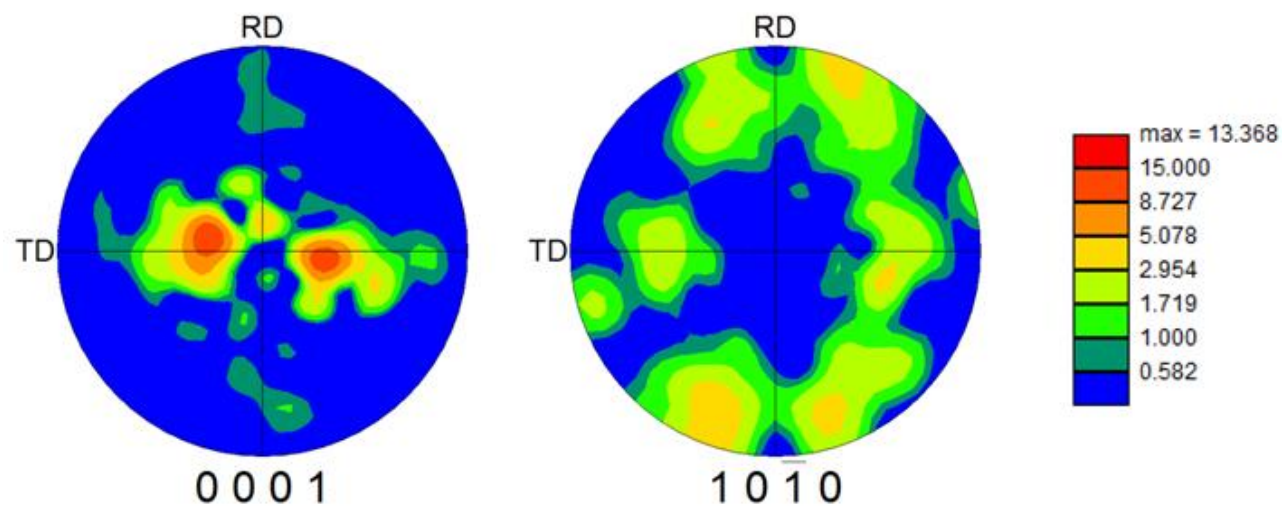
(b)

\section{Experiment}
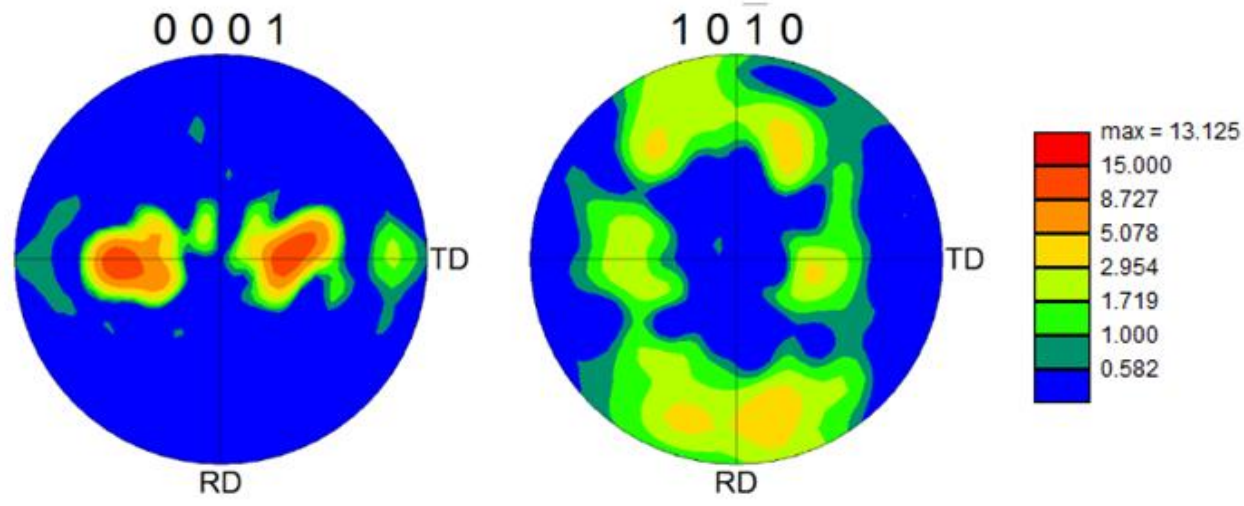

Simulation
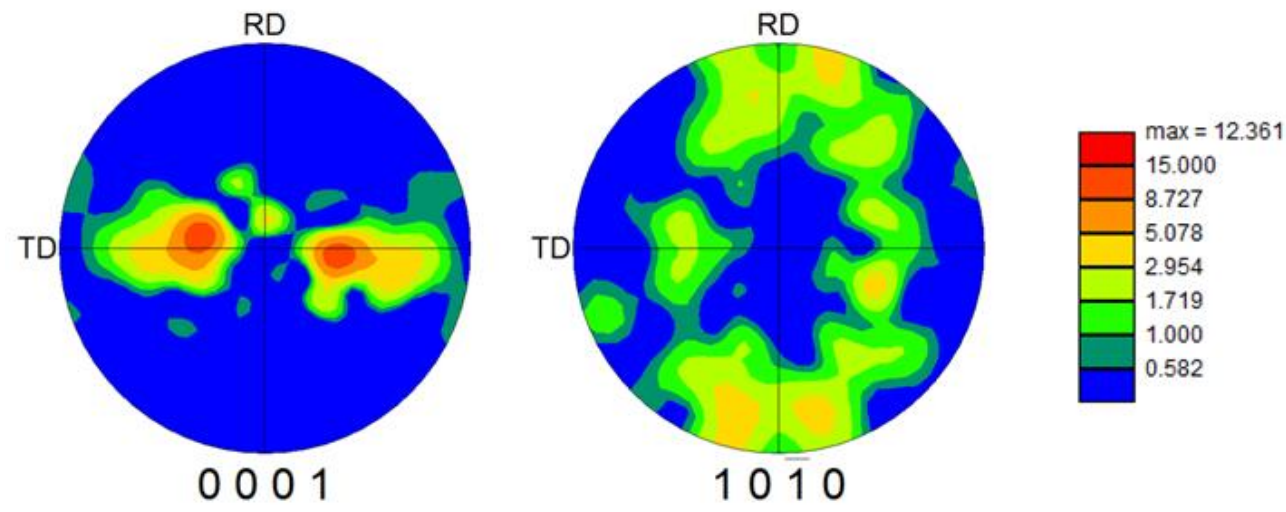

Fig. 11 (0001) and (10 $\overline{1} 0)$ pole figures under TC loading in TD obtained from experiment and simulation. Results are at points (a) C and (b) D shown in Fig. 7(b). 
(a)

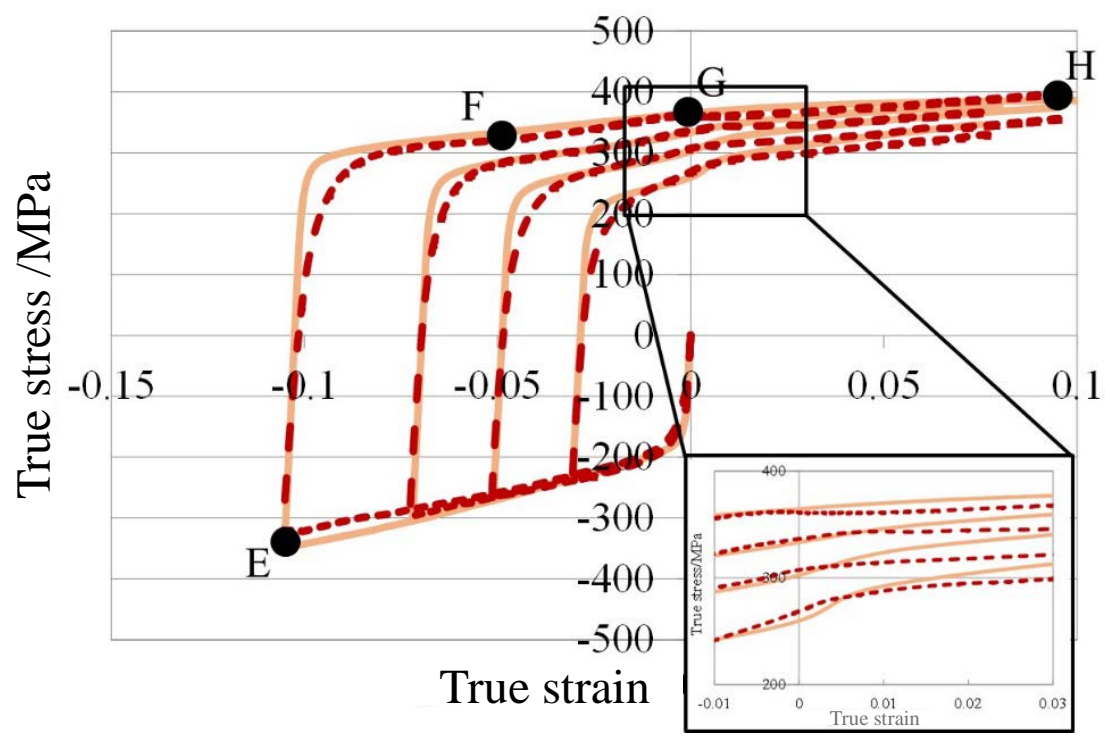

(b)

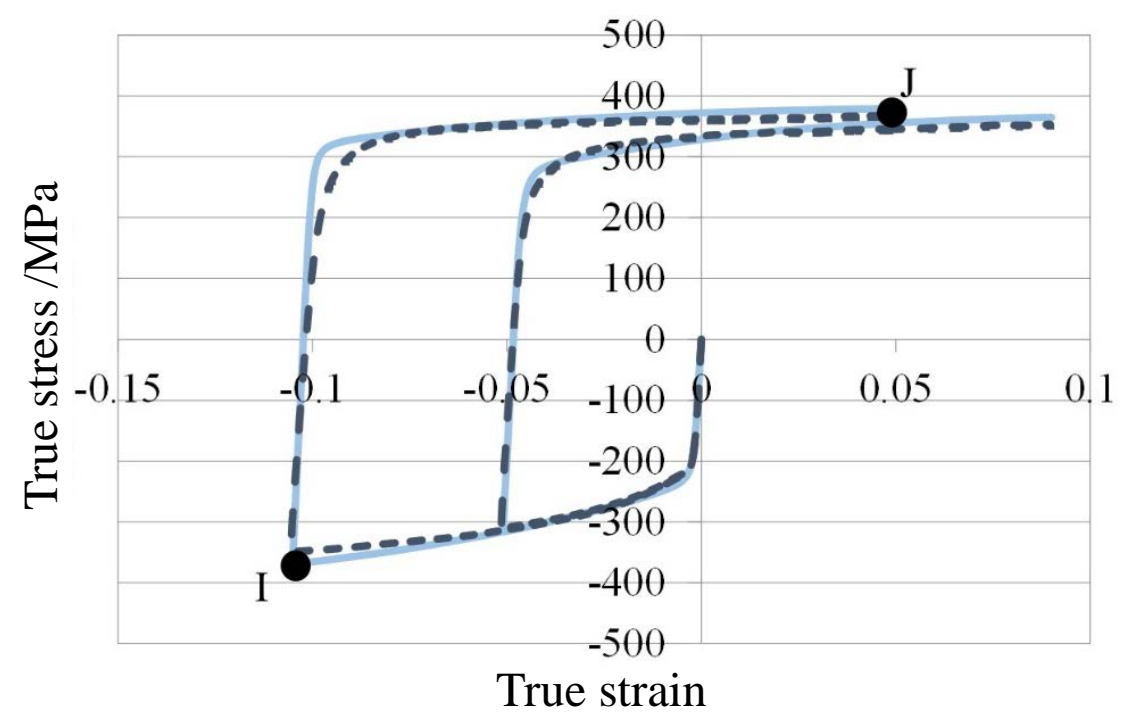

Fig. 12 Stress-strain curves under CT loading in (a) RD and (b) TD obtained from experiment (dotted lines) [Yi et al., 2016] and simulation (solid lines). 
(a)

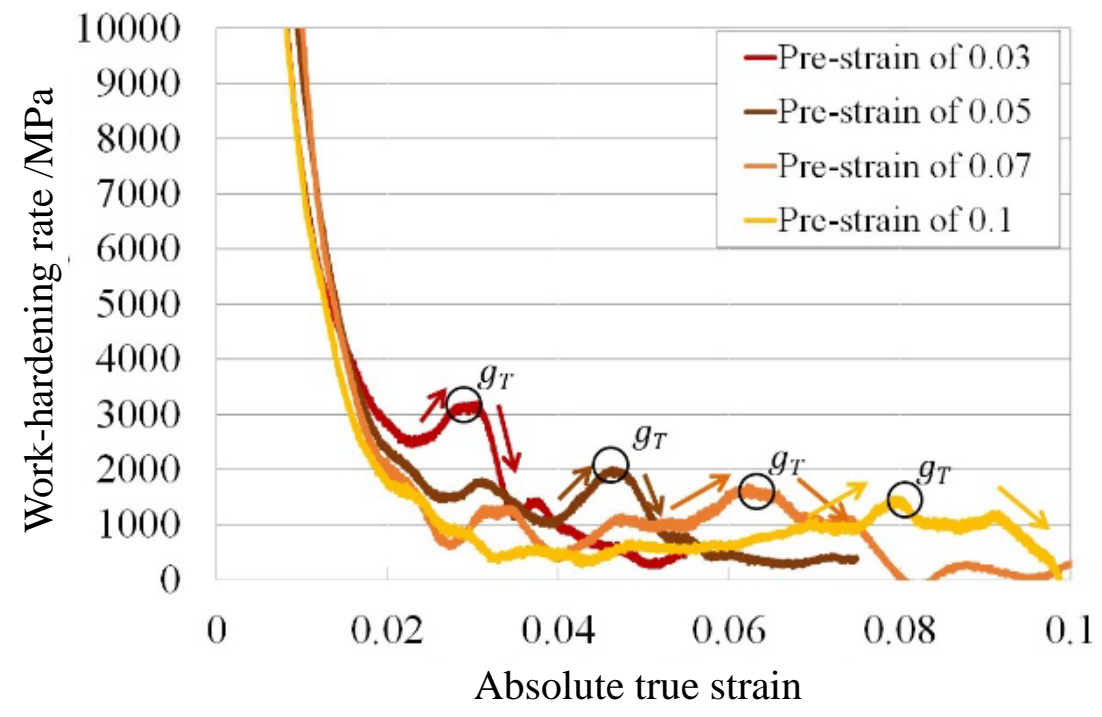

(b)

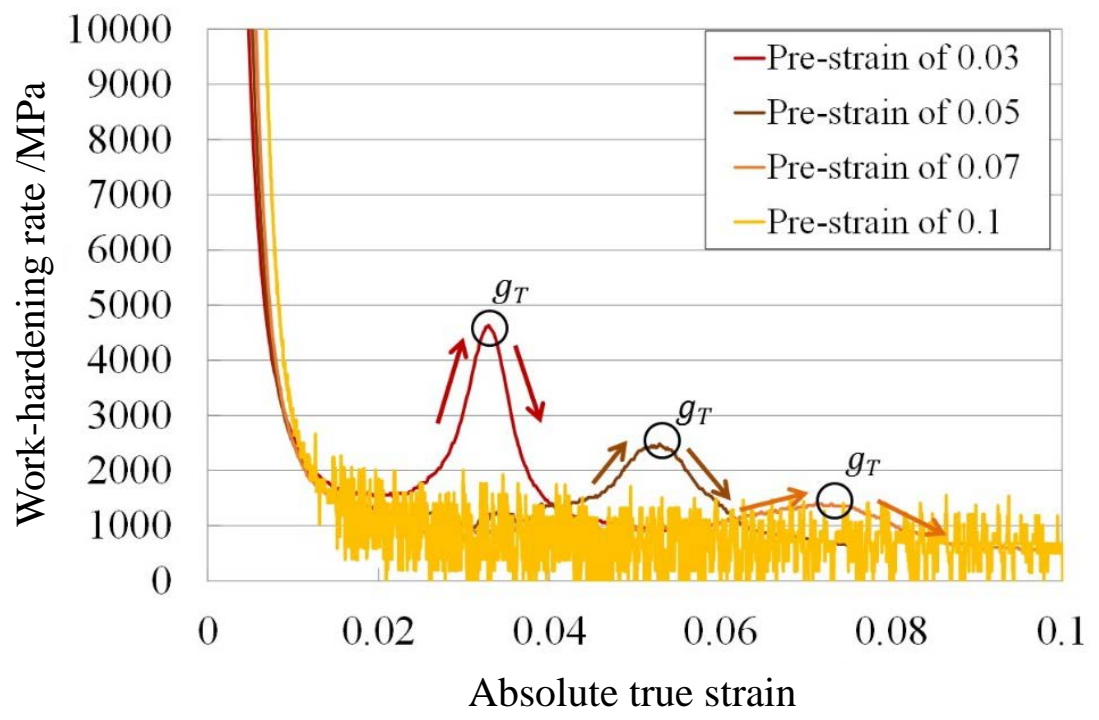

Fig. 13 Variations of work-hardening rate after stress reversal under CT loading in RD obtained from (a) experiment [Yi et al., 2016] and (b) simulation. 
(a)

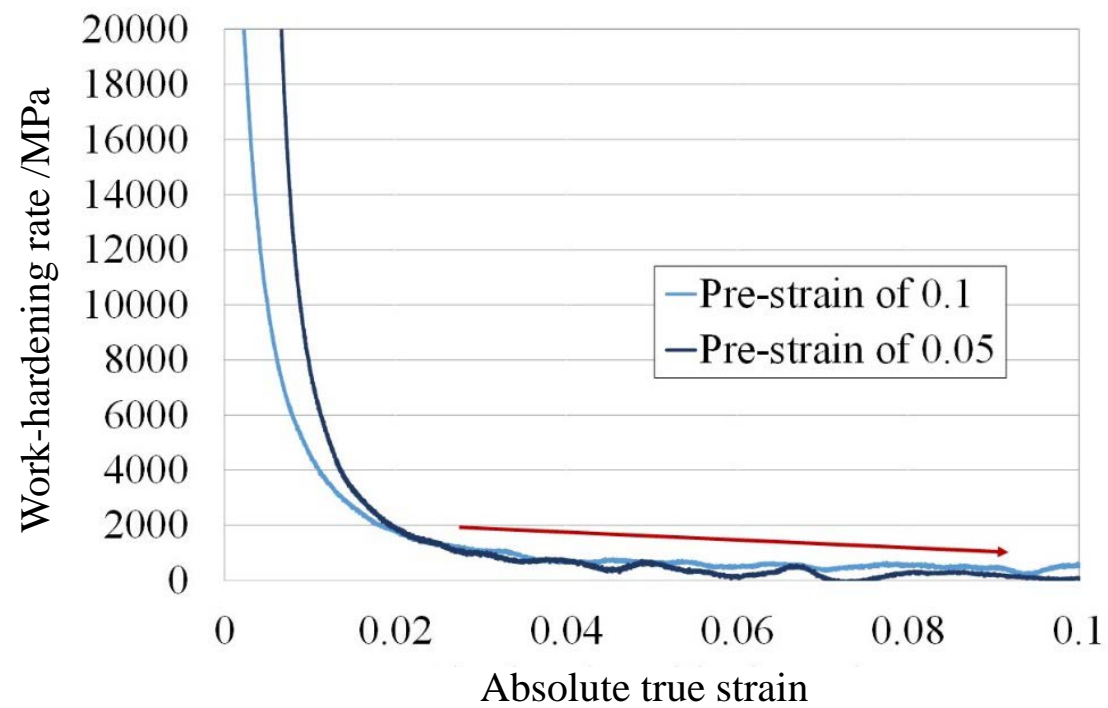

(b)

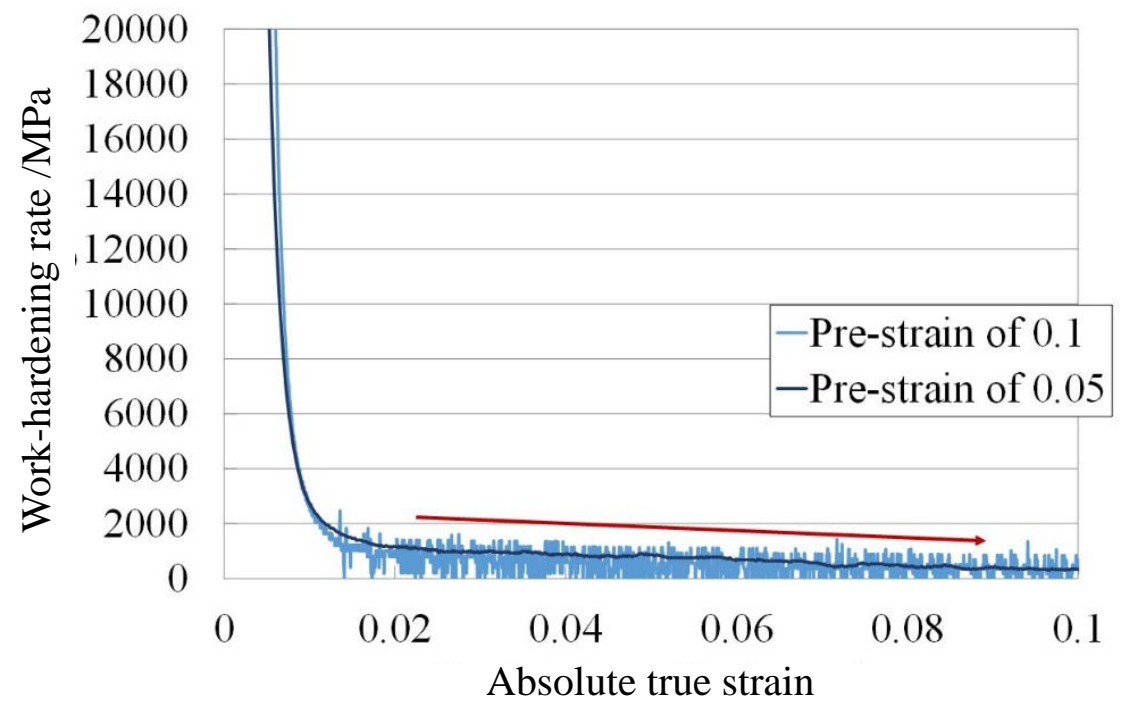

Fig. 14 Variations of work-hardening rate after stress reversal under CT loading in TD obtained from (a) experiment [Yi et al., 2016] and (b) simulation. 


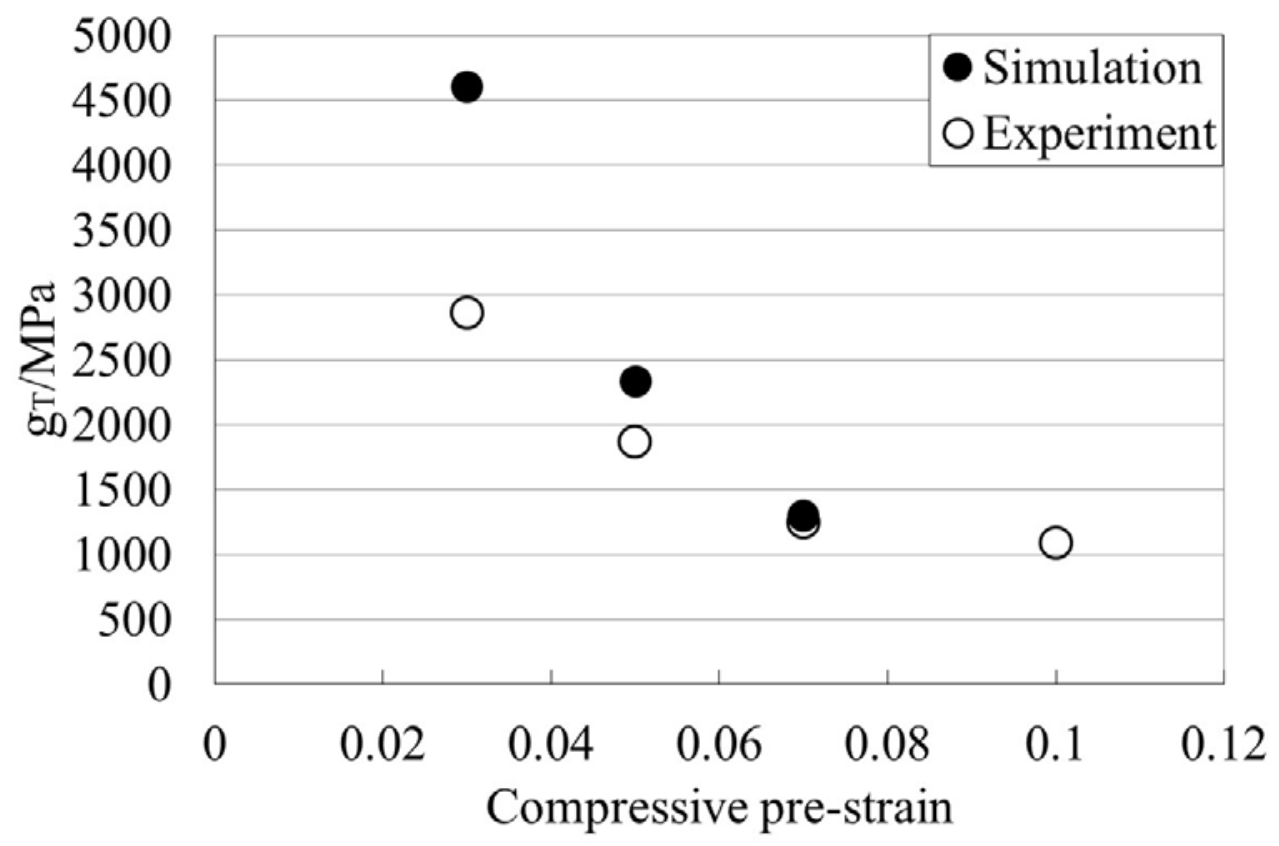

Fig. 15 Relationship between $g_{T}$ and compressive pre-strain obtained from experiment and simulation. 
(a)

\section{Experiment}
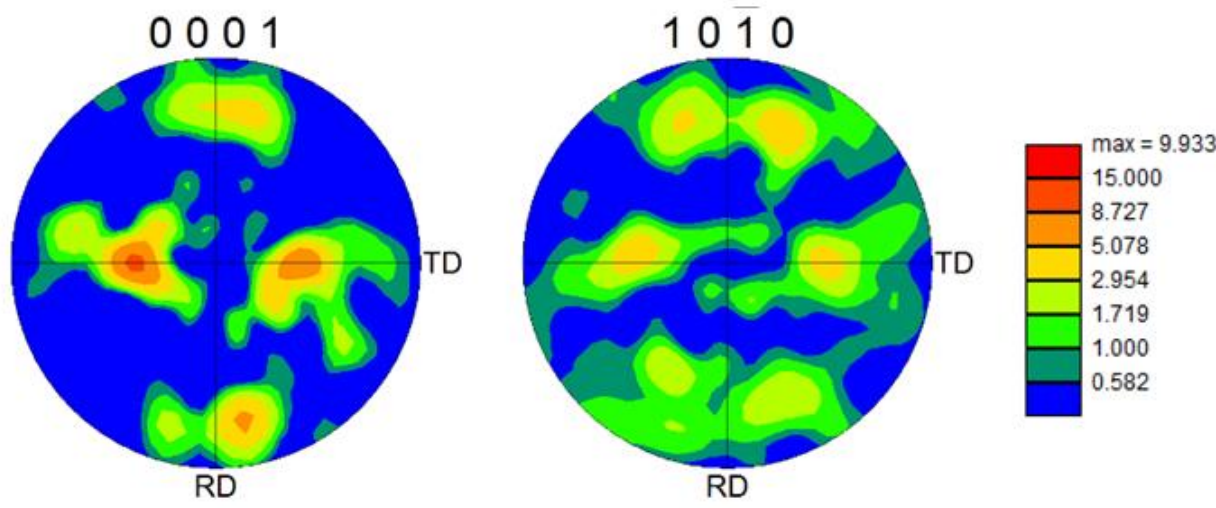

Simulation
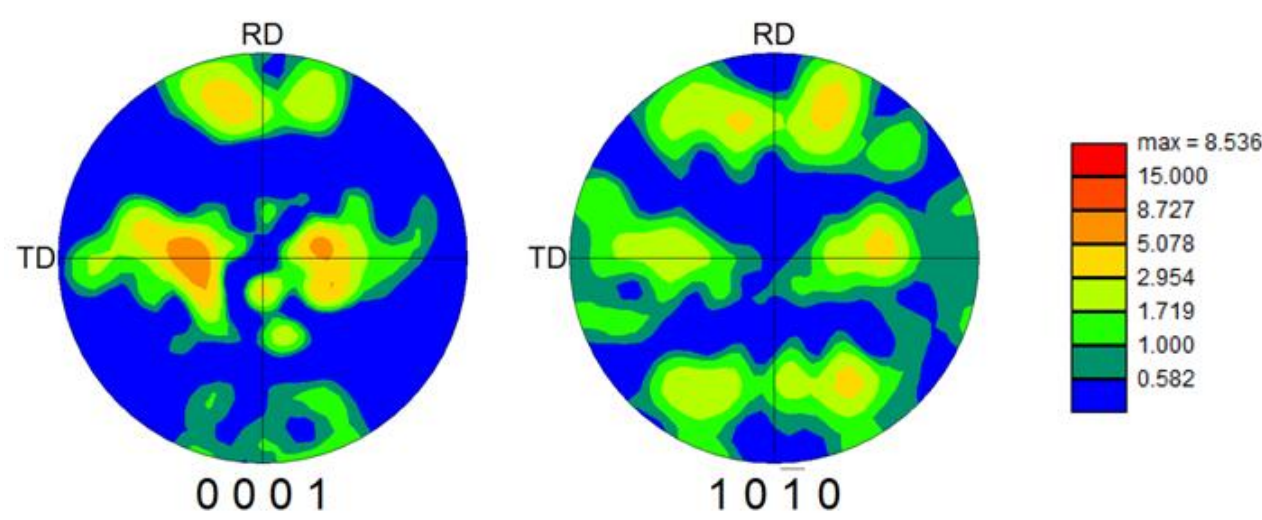
(b)

\section{Experiment}
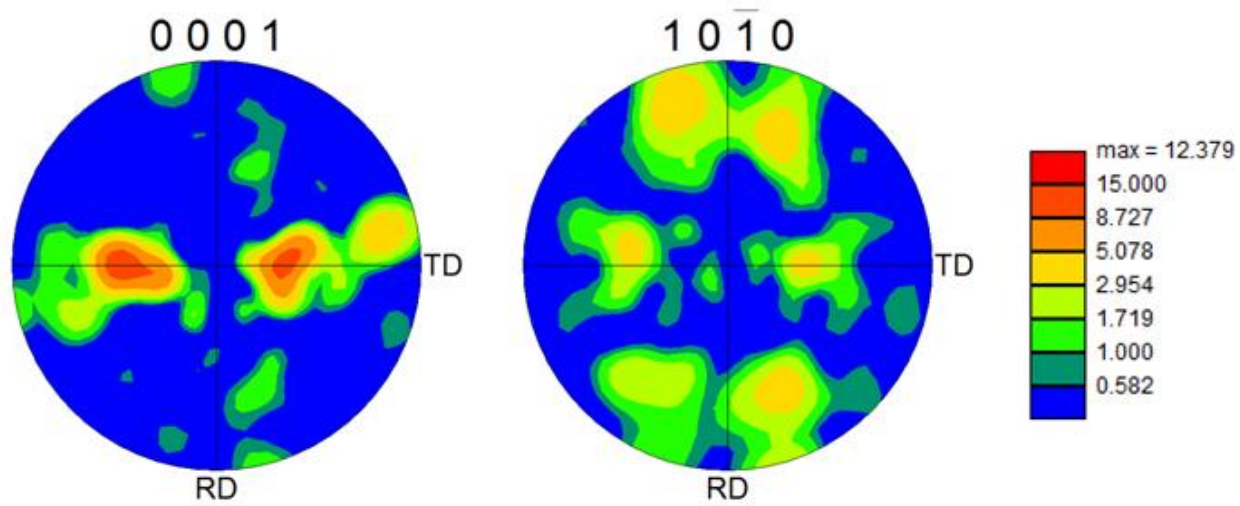

Simulation
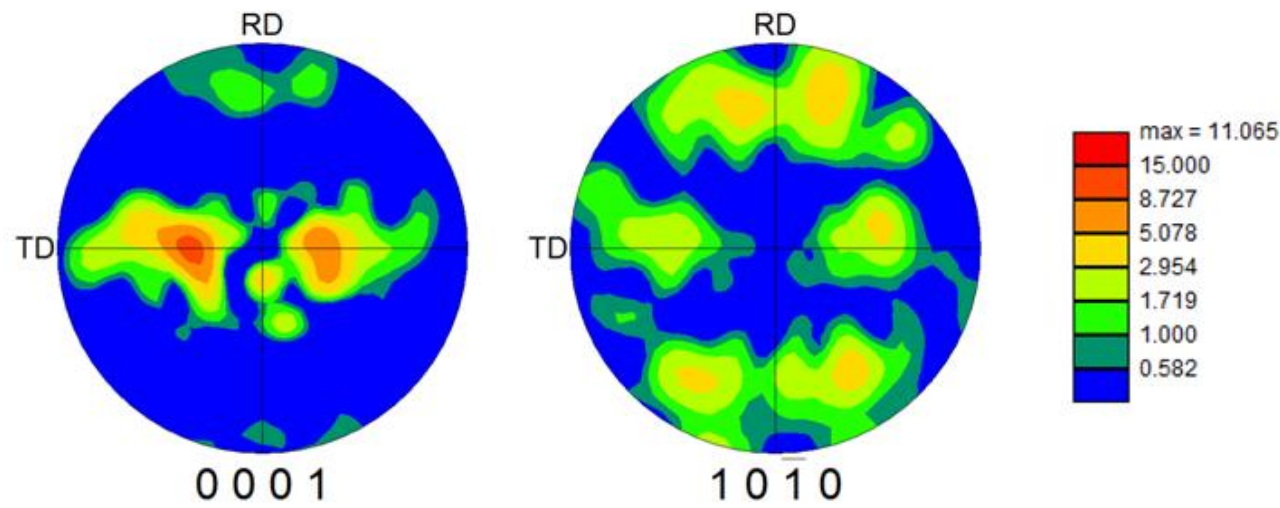
(c)

Experiment
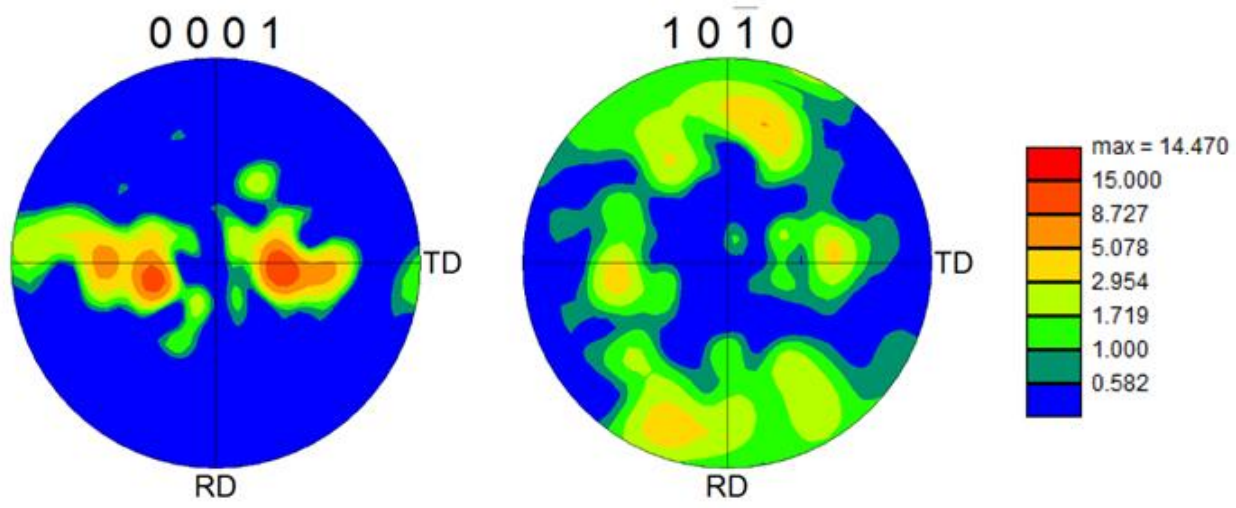

Simulation
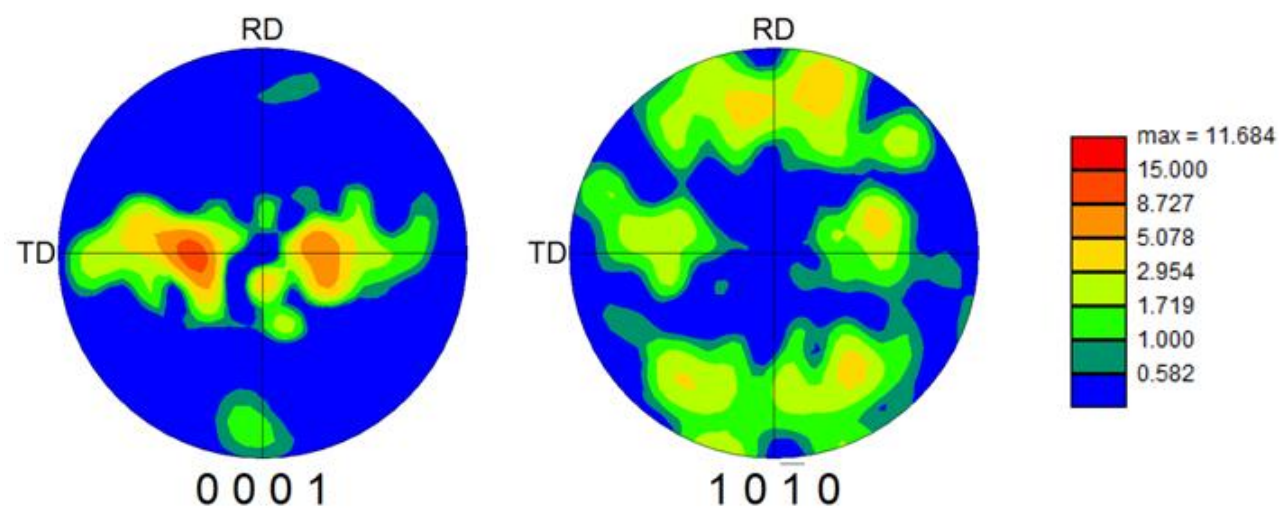
(d)

\section{Experiment}
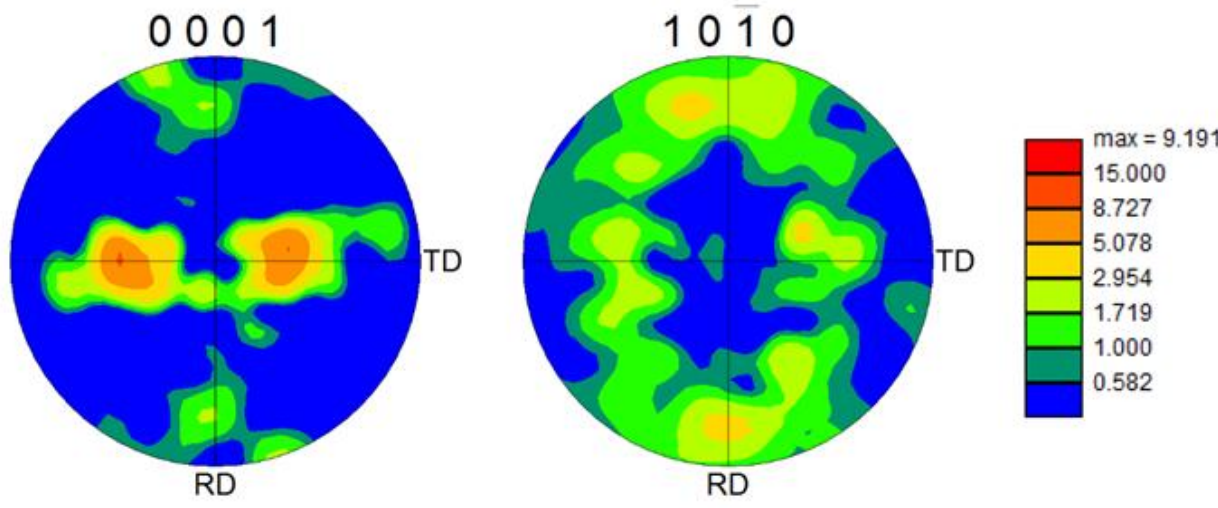

Simulation
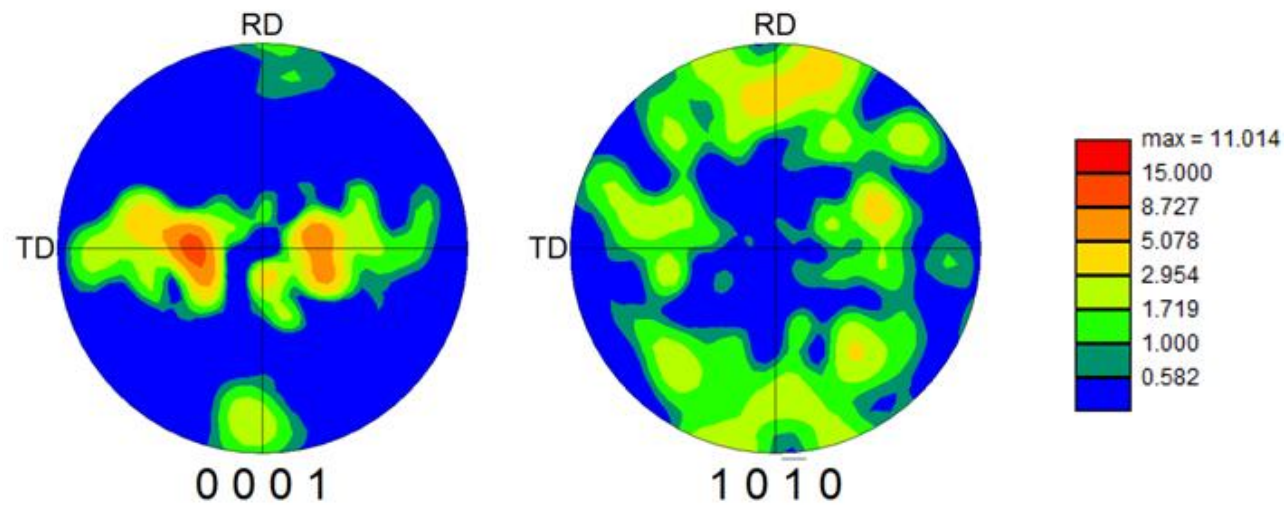

Fig. 16 (0001) and (10 $\overline{1} 0)$ pole figures obtained under CT loading in RD obtained from experiment [Yi et al., 2016] and simulation. Results are at points (a) E, (b) F, (c) G, and (d) H shown in Fig. 12(a). 
(a)

\section{Experiment}
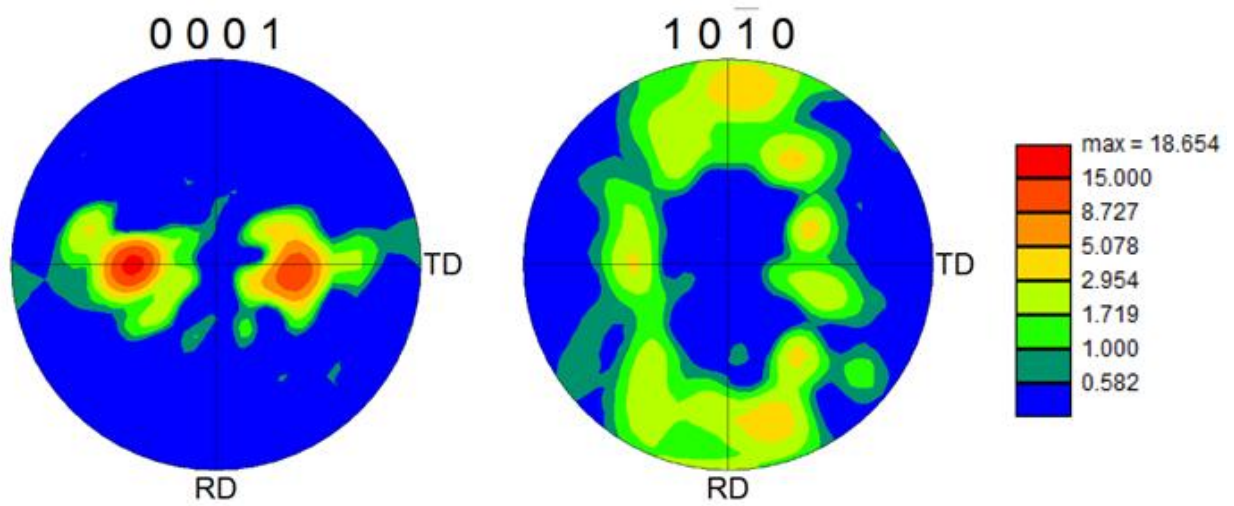

Simulation
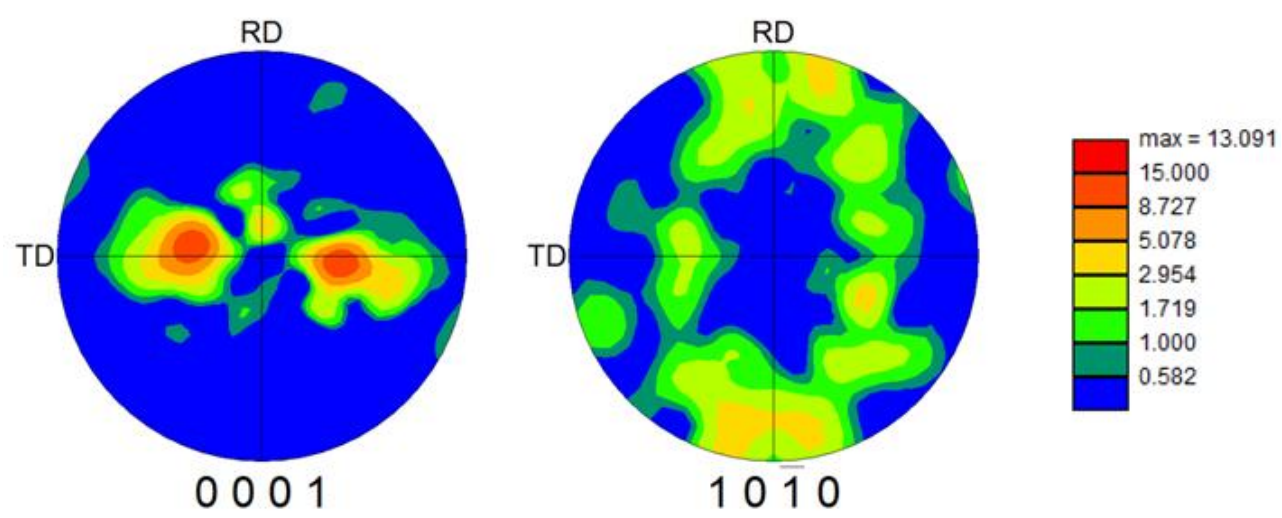
(b)

\section{Experiment}
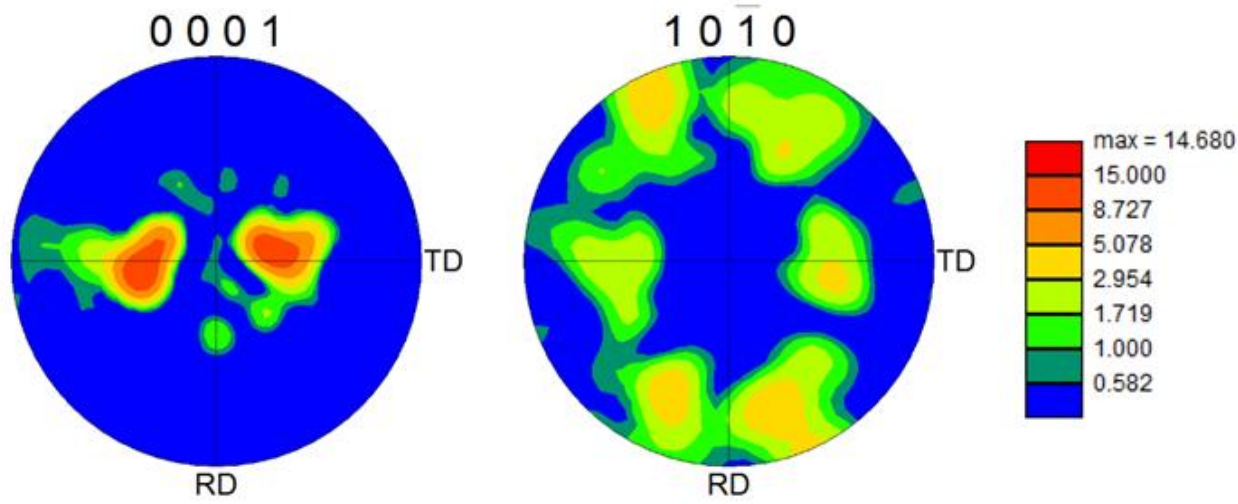

Simulation
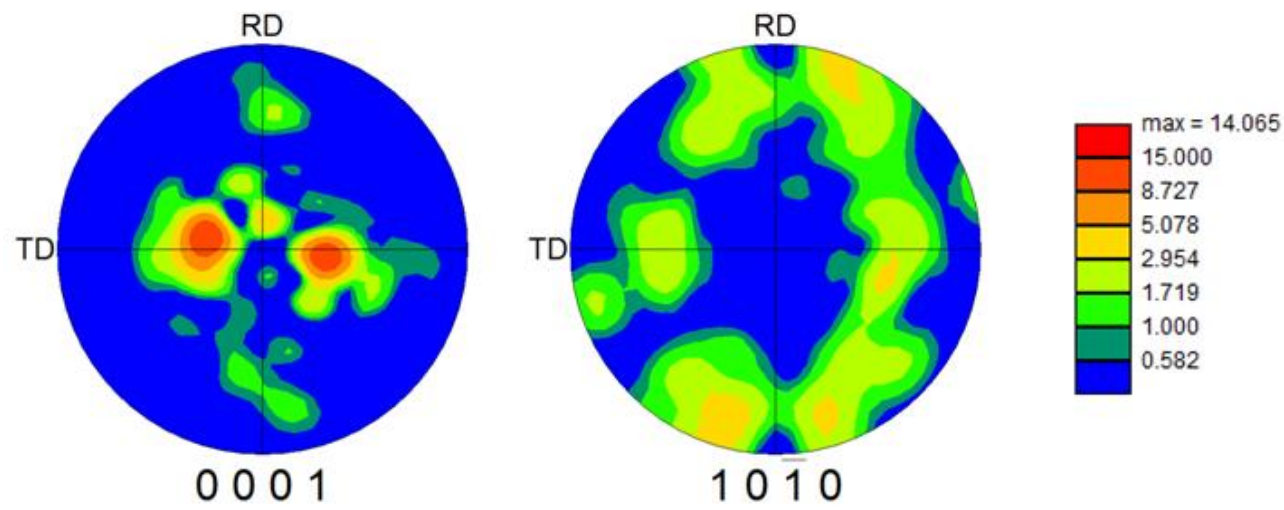

Fig. 17 (0001) and (10 $\overline{1} 0)$ pole figures obtained under CT loading in TD obtained from experiment and simulation. Results are at points (a) I and (b) J shown in Fig. 12(b). 


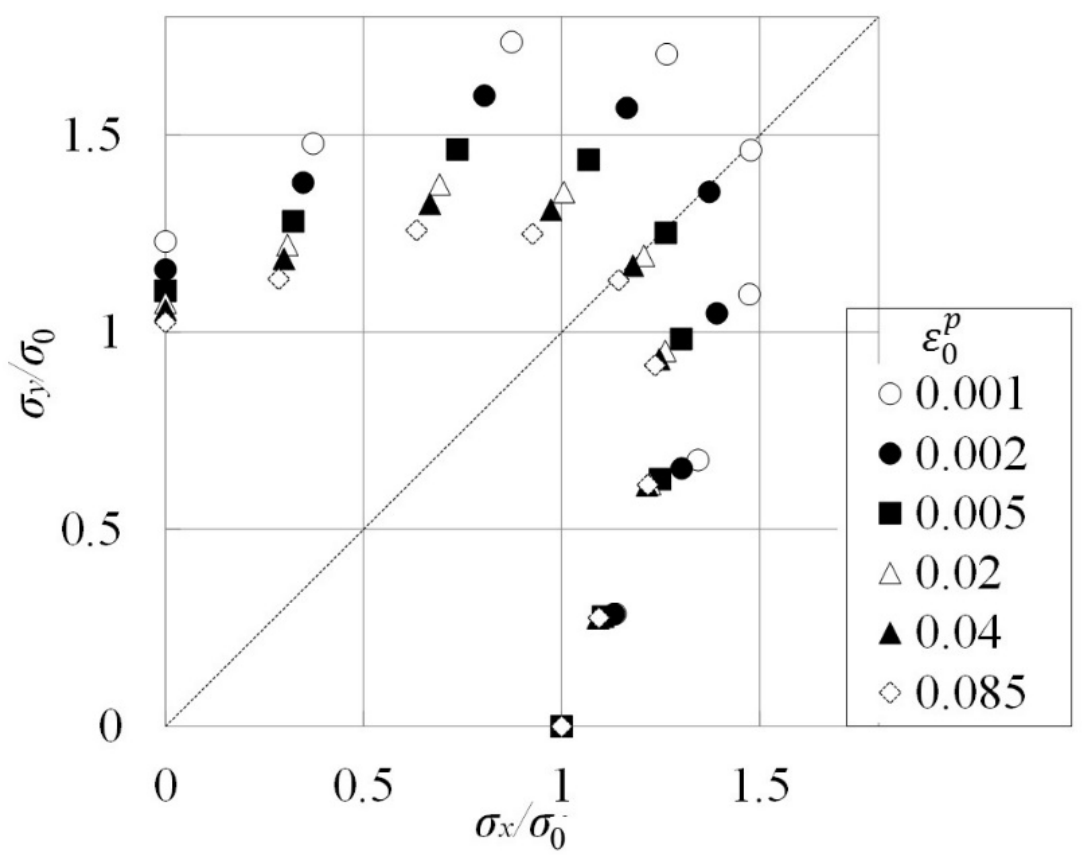

Fig. 18 Normalized contours of plastic work obtained from simulation. 


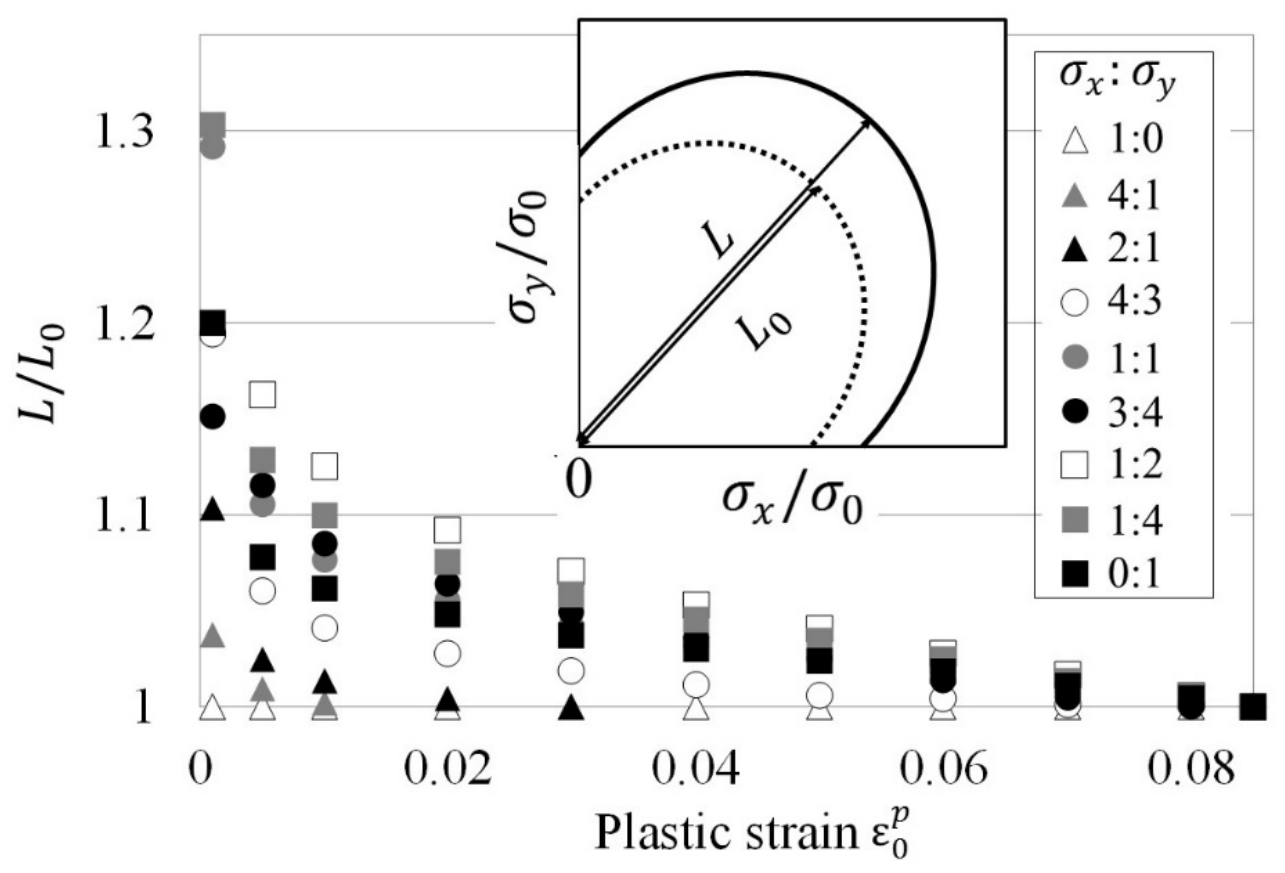

Fig. 19 Variations of shape ratio of stress points $\left(L / L_{0}\right)$ in each loading direction as a function of plastic strain. 
(a)

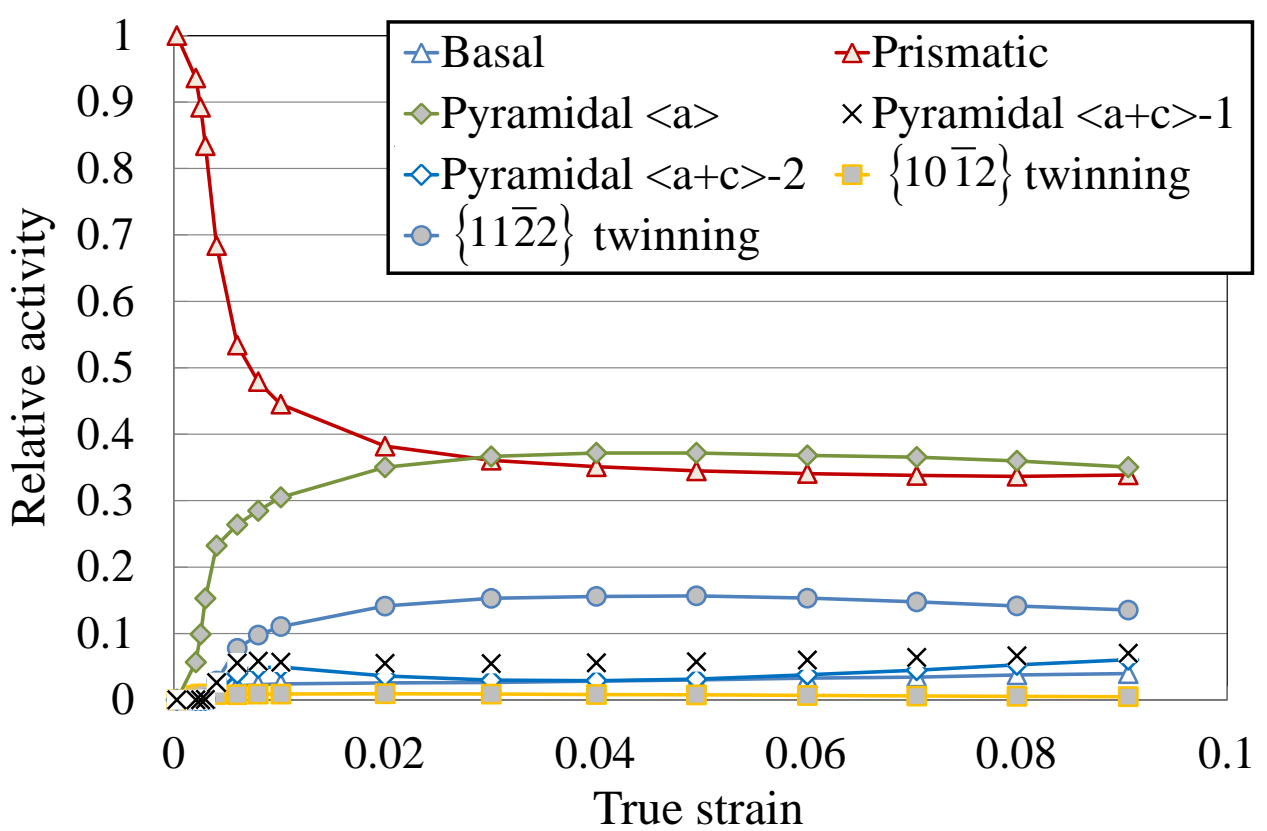

(b)

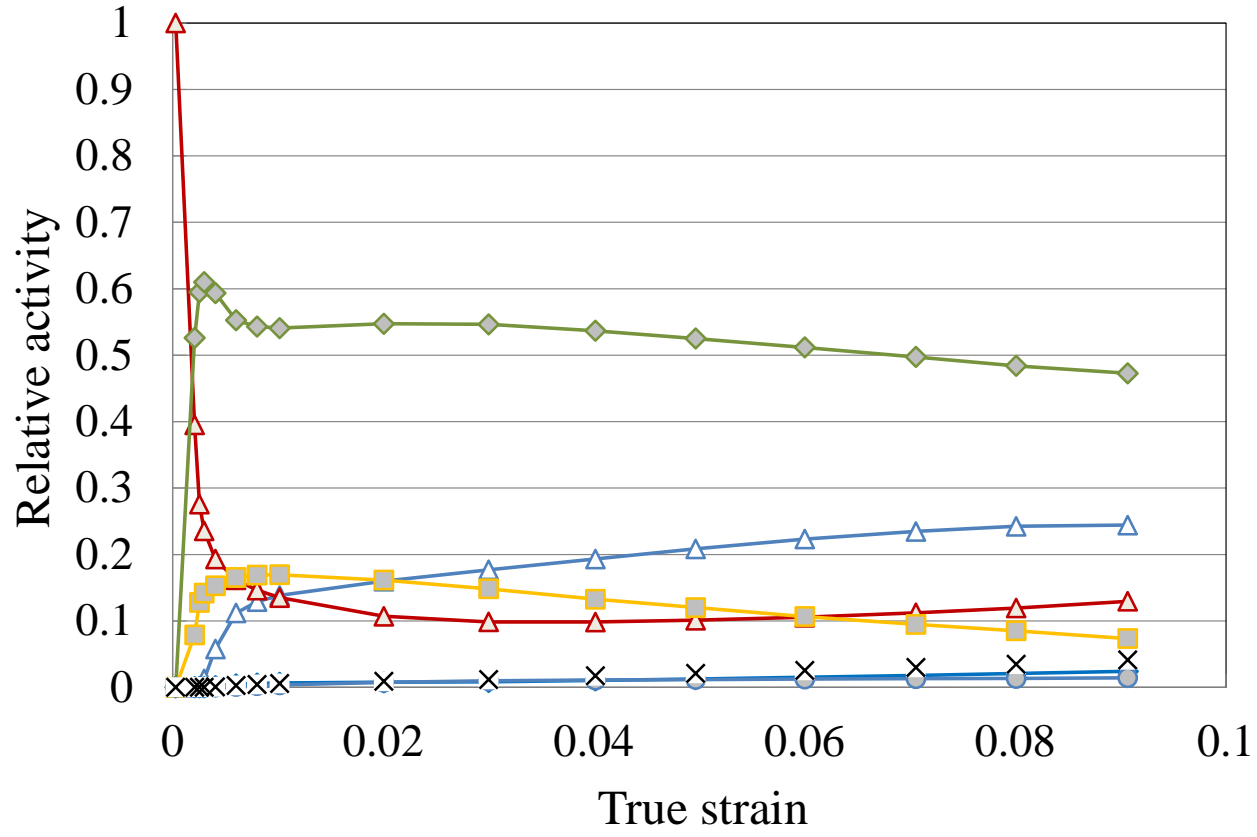

Fig. 20 Evolution of relative activity during tension in (a) RD and (b) TD. 


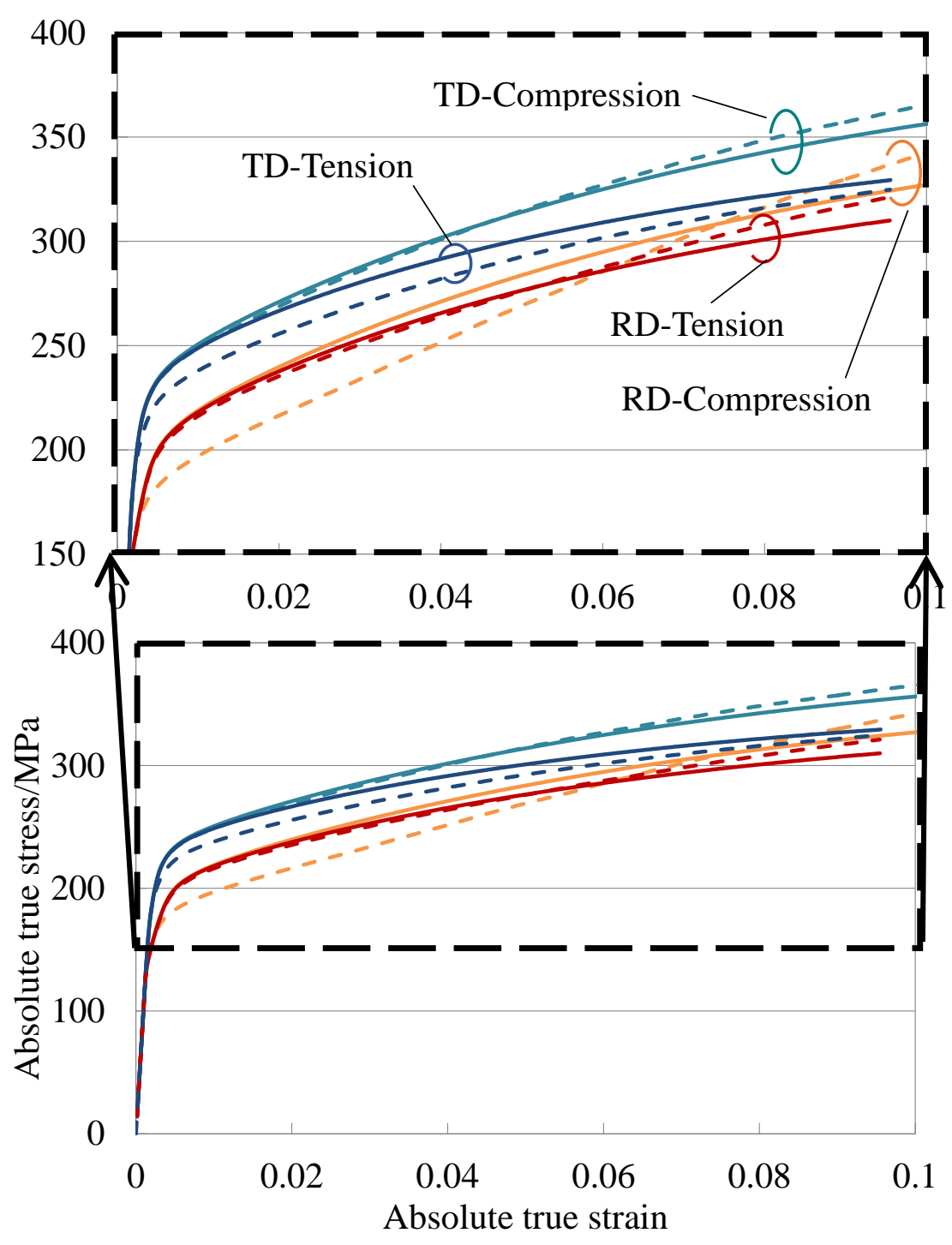

Fig. 21 Absolute stress-absolute strain curves under tension and compression obtained with (dotted lines) and without (solid lines) considering twinning activity. 


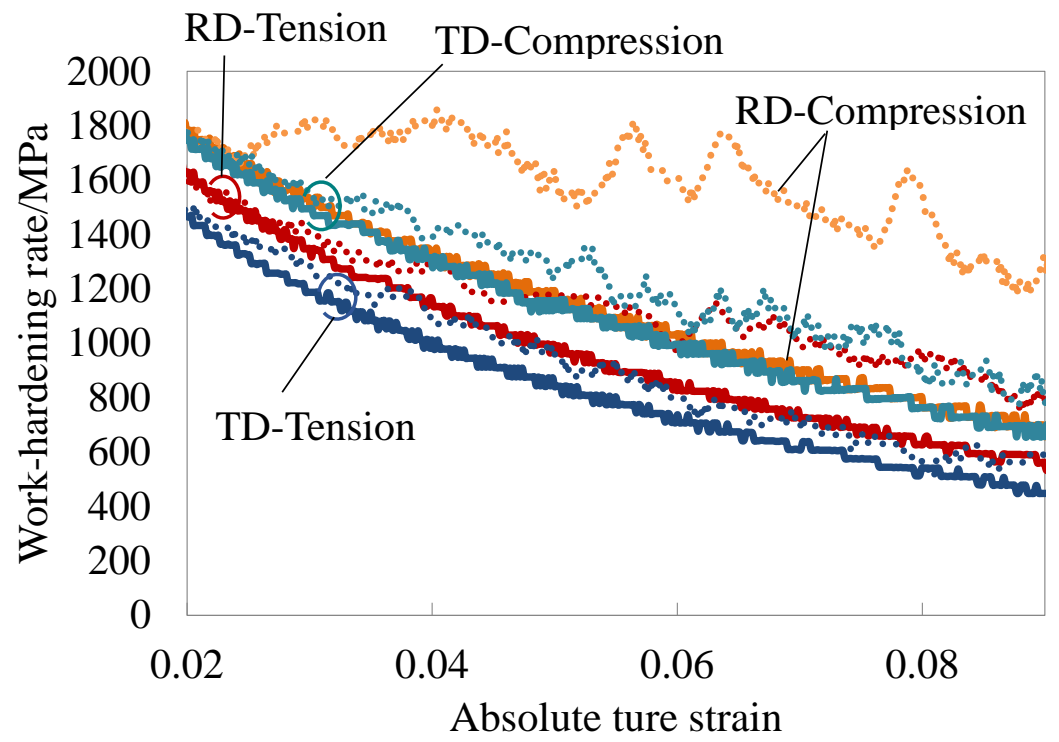

Fig. 22 Evolution of work-hardening rates under tension and compression obtained with (dotted lines) and without (solid lines) considering twinning activity. 
(a)

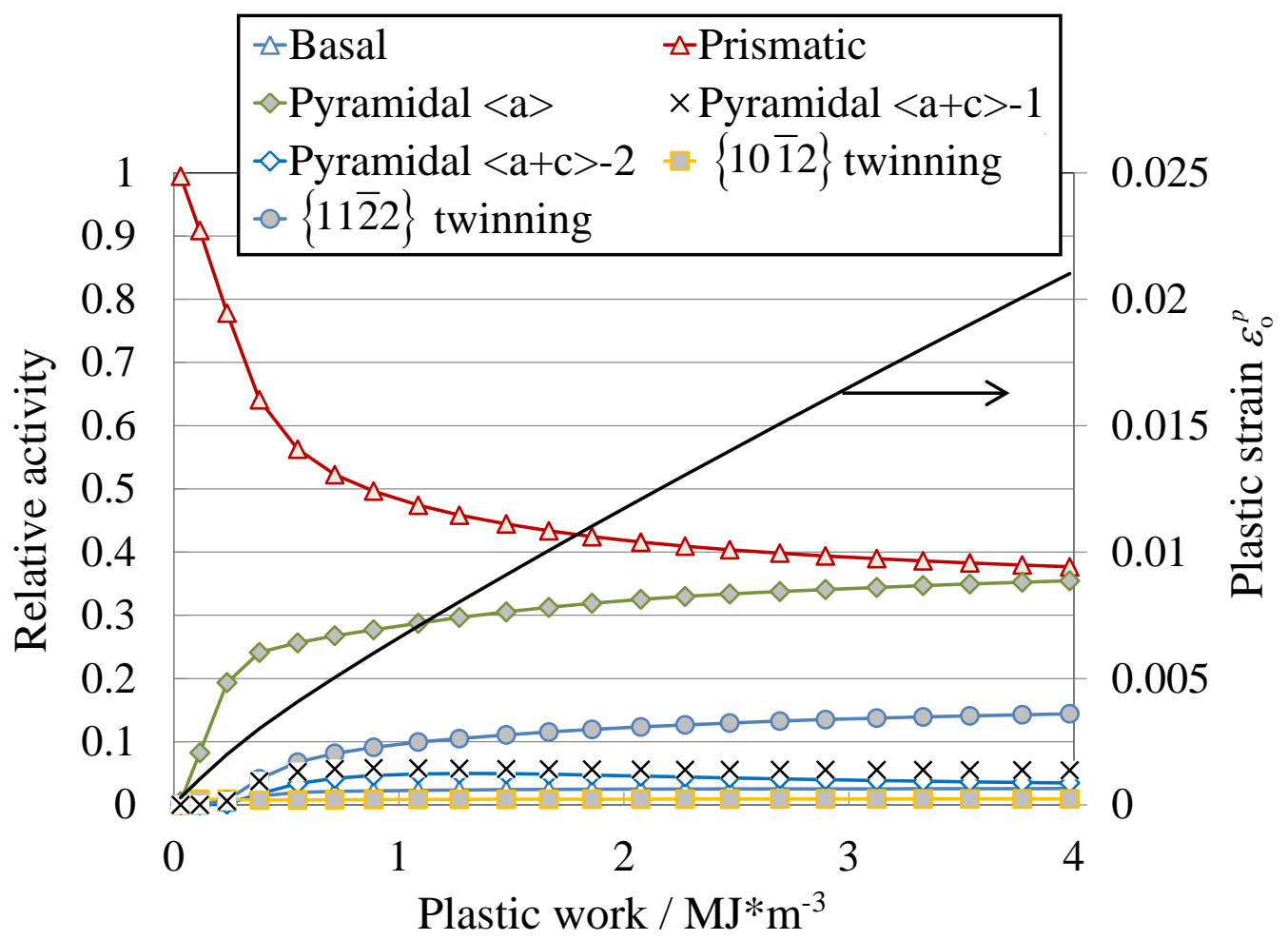

(b)

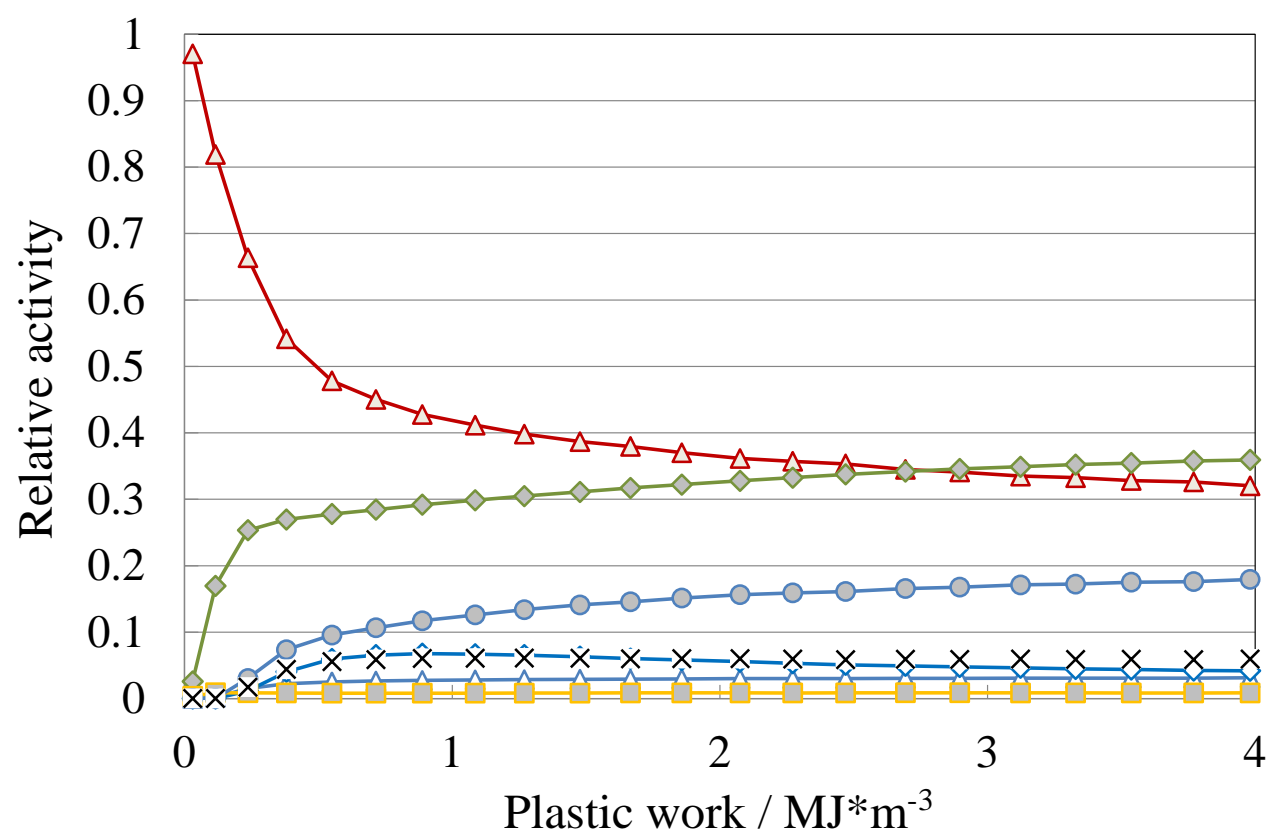


(c)

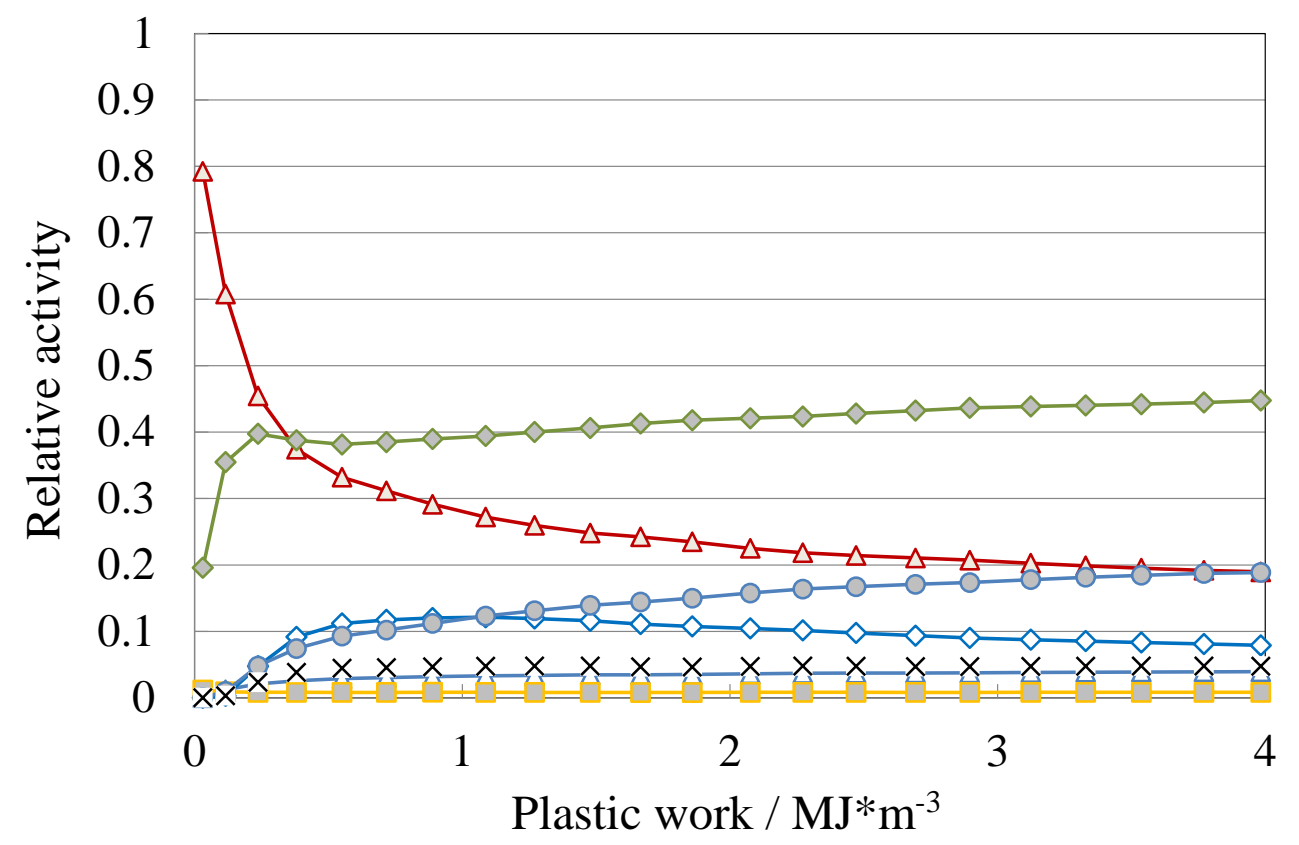

(d)

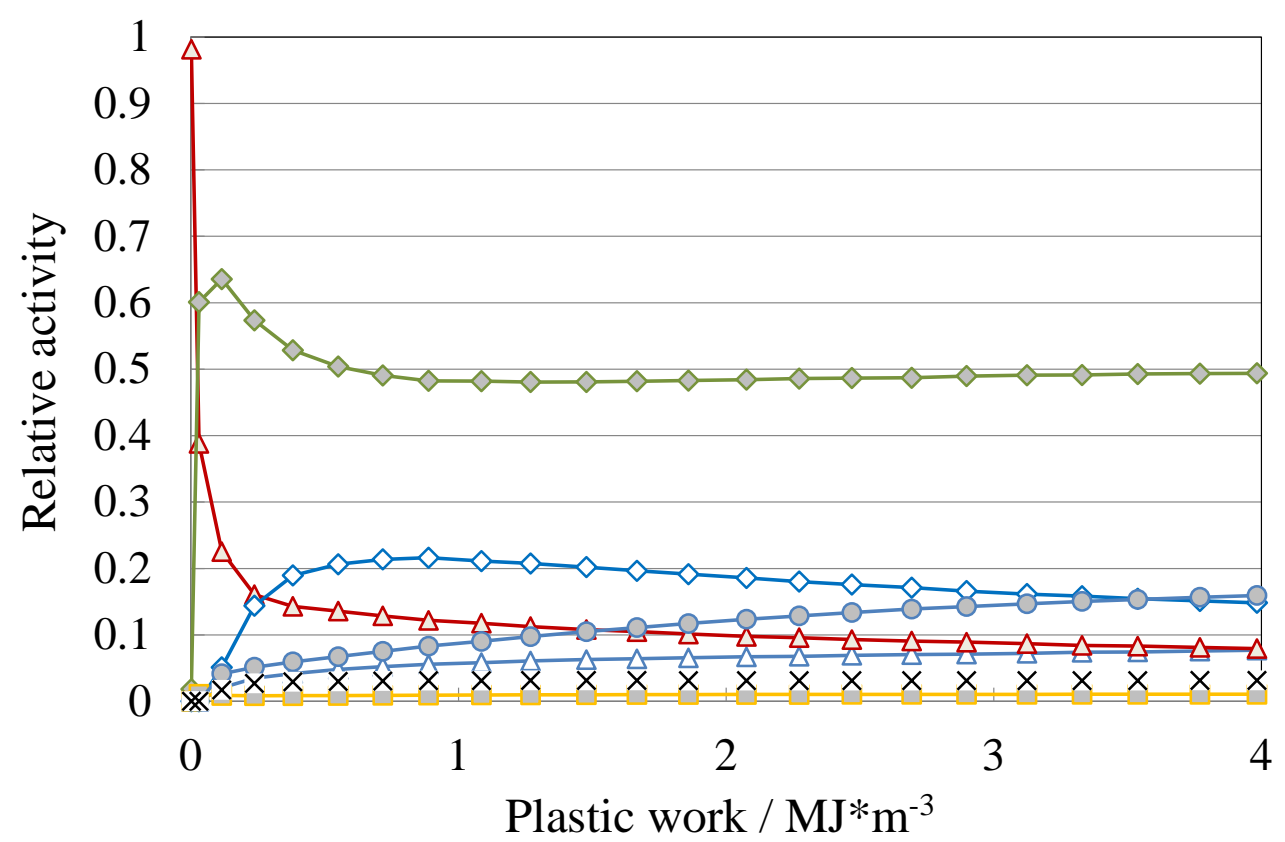


(e)

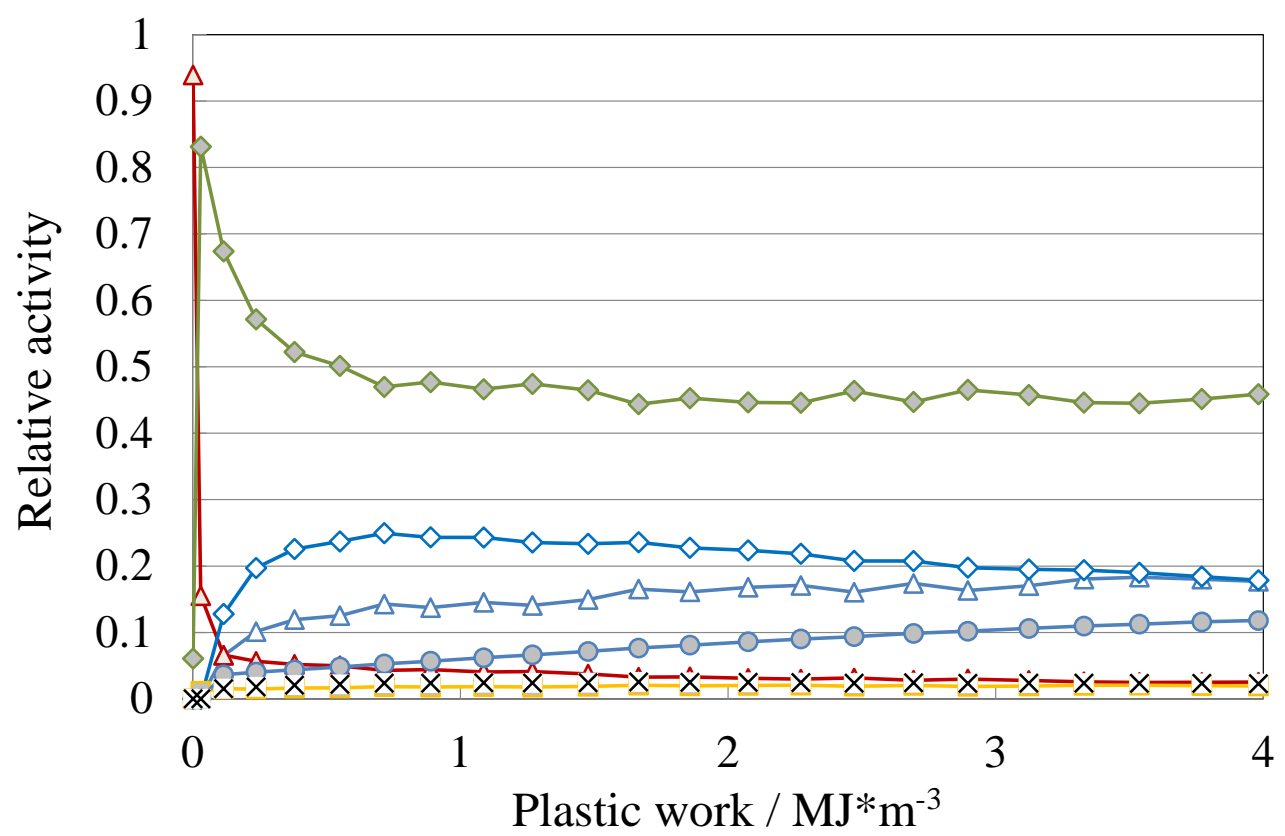

Fig. 23 Evolution of relative activities as a function of plastic work for biaxial stress ratios $\sigma_{x}: \sigma_{y}=$ (a) $1: 0$, (b) $4: 1$, (c) $2: 1$, (d) $4: 3$, and (e) $1: 1$. 
(a)

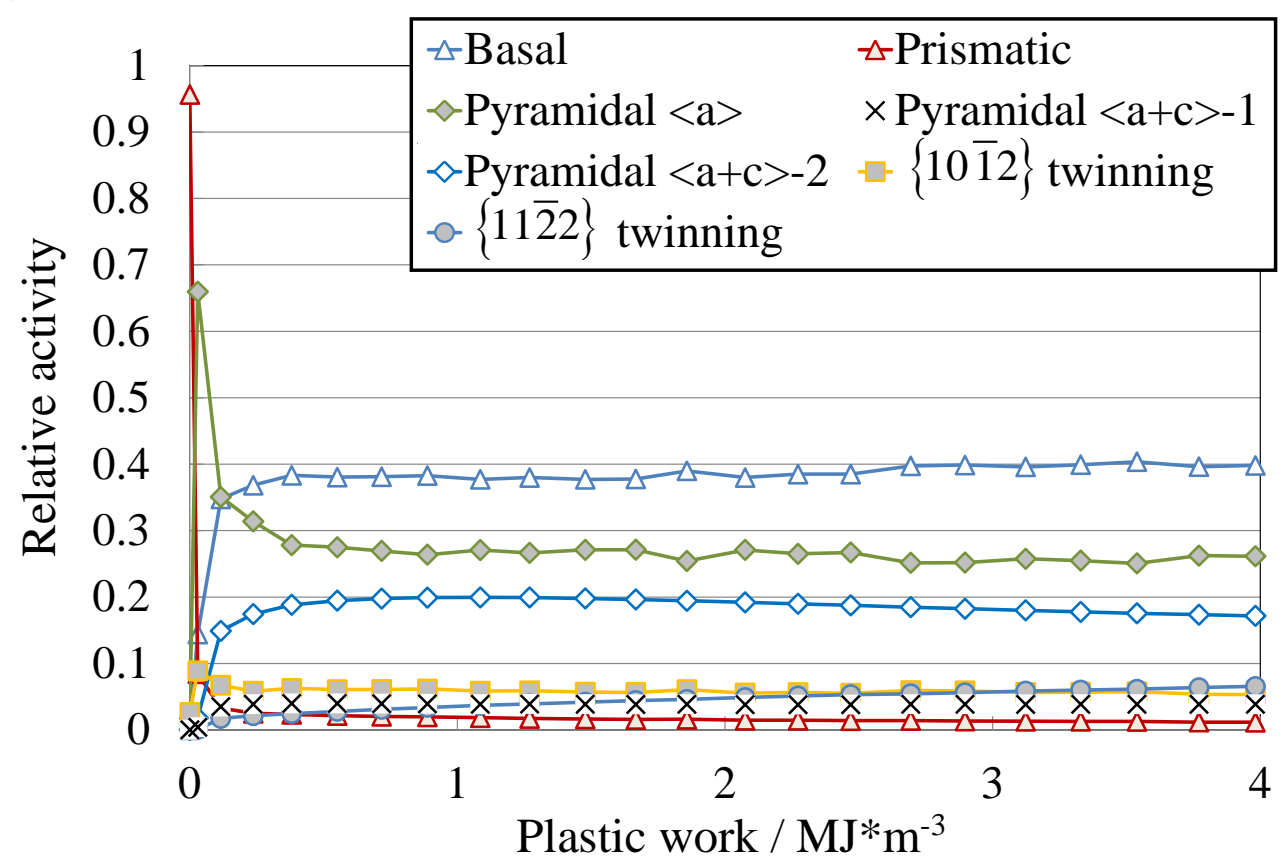

(b)

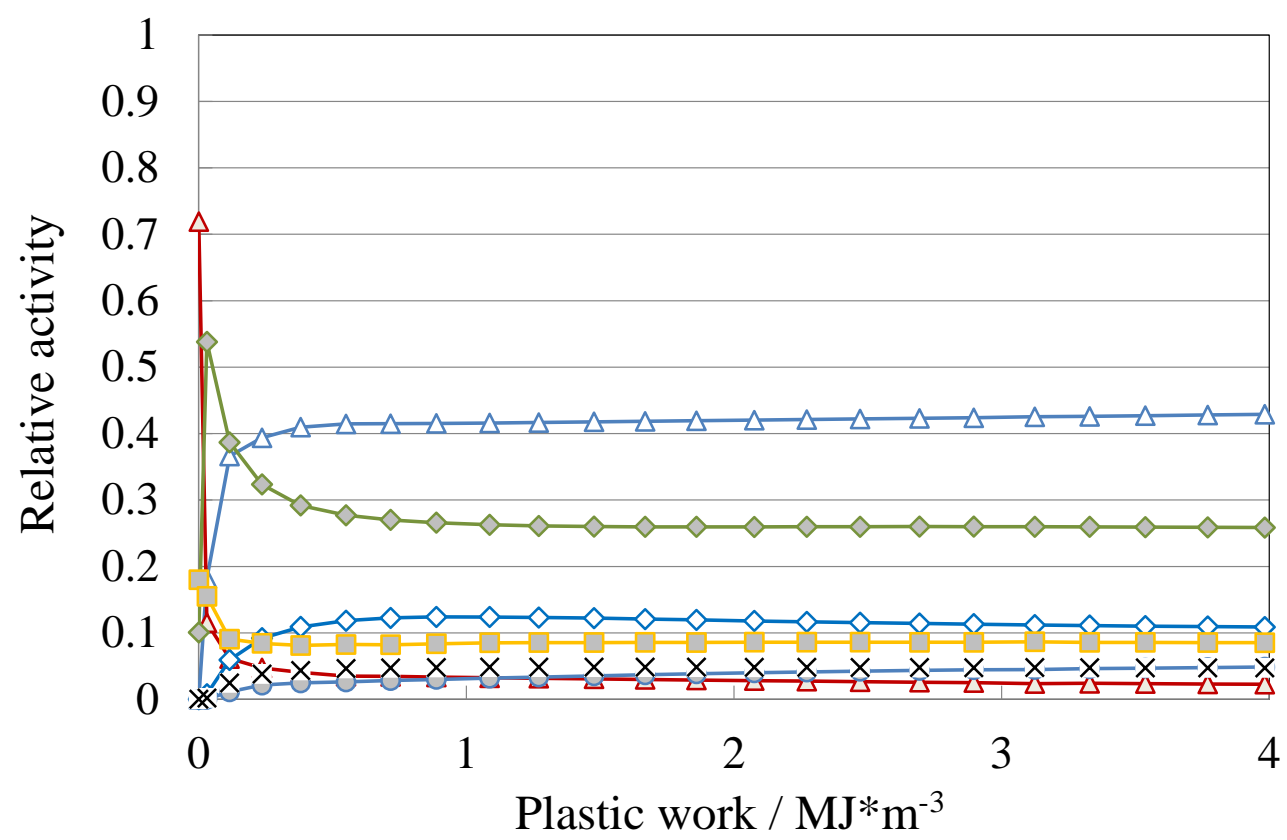


(c)

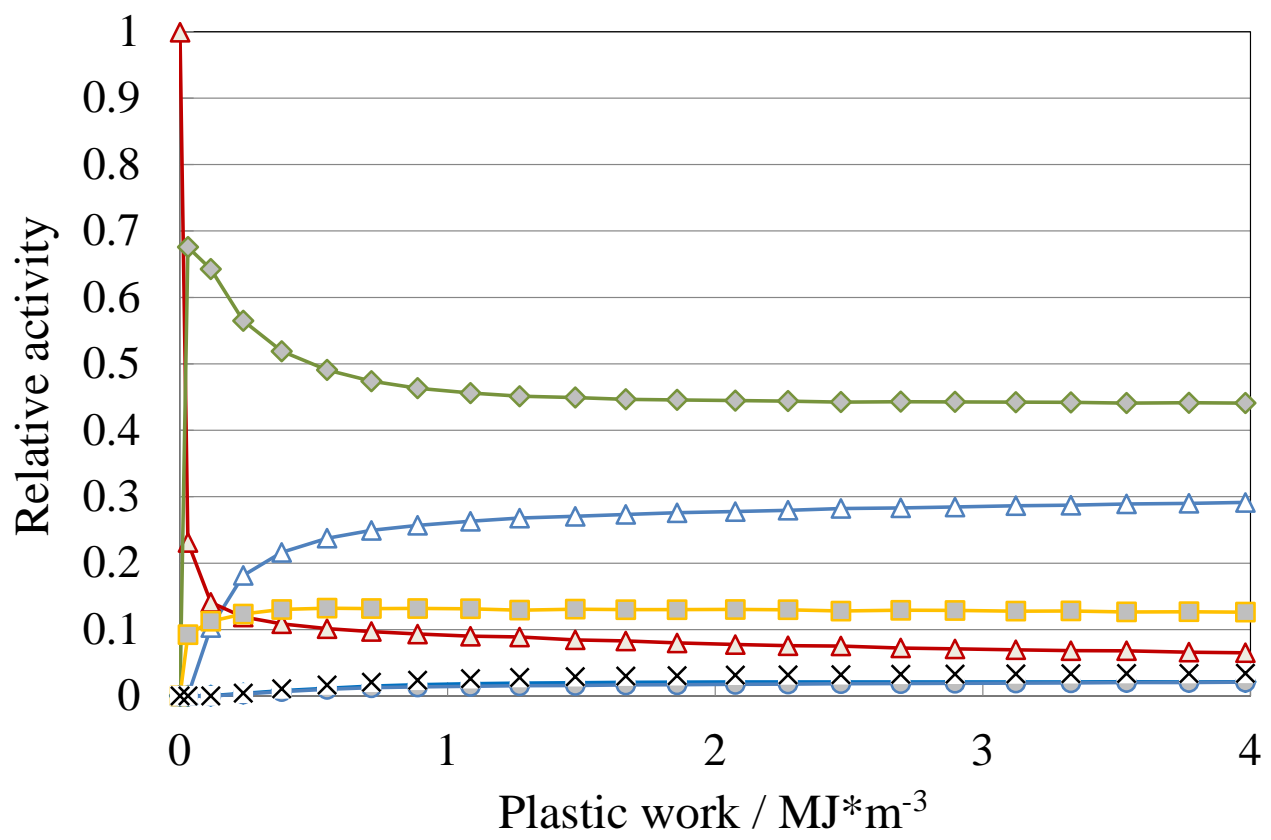

(d)

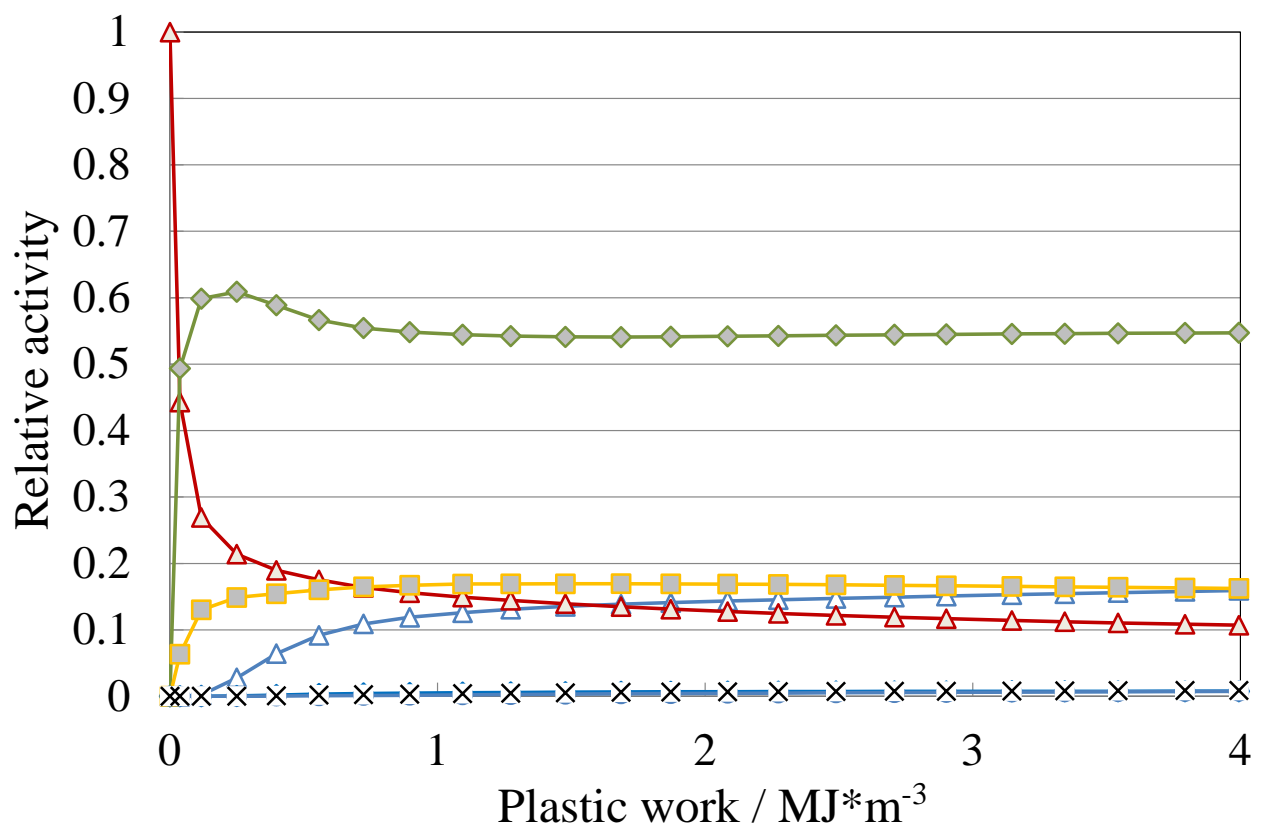

Fig. 24 Evolution of relative activities as a function of plastic work for biaxial stress ratios $\sigma_{x}: \sigma_{y}=$ (a) $3: 4$, (b) $1: 2$, (c) $1: 4$, and (d) 0:1. 


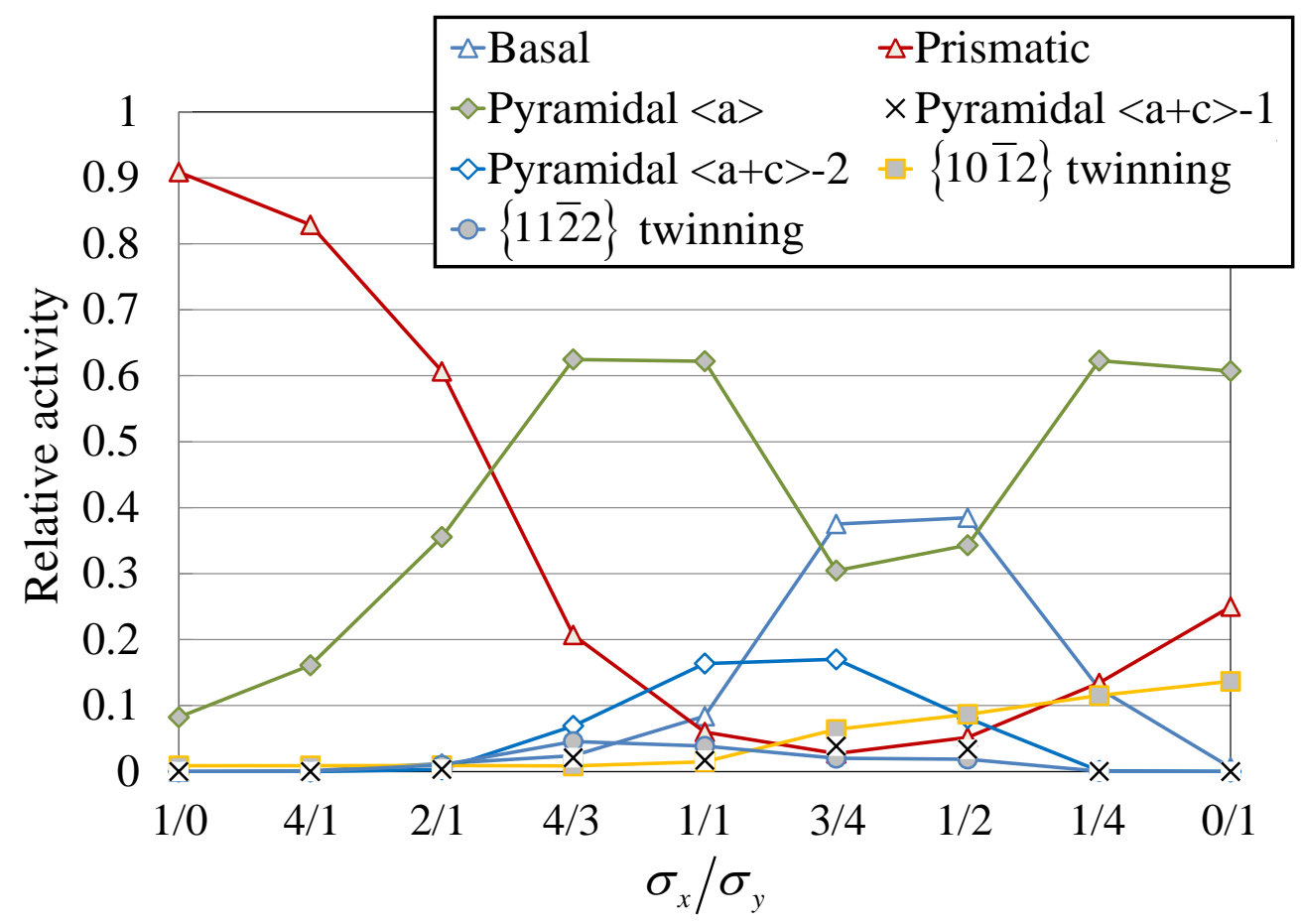

Fig. 25 Relationship between relative activity and biaxial stress ratio at plastic strain of 0.001 . 
(a)

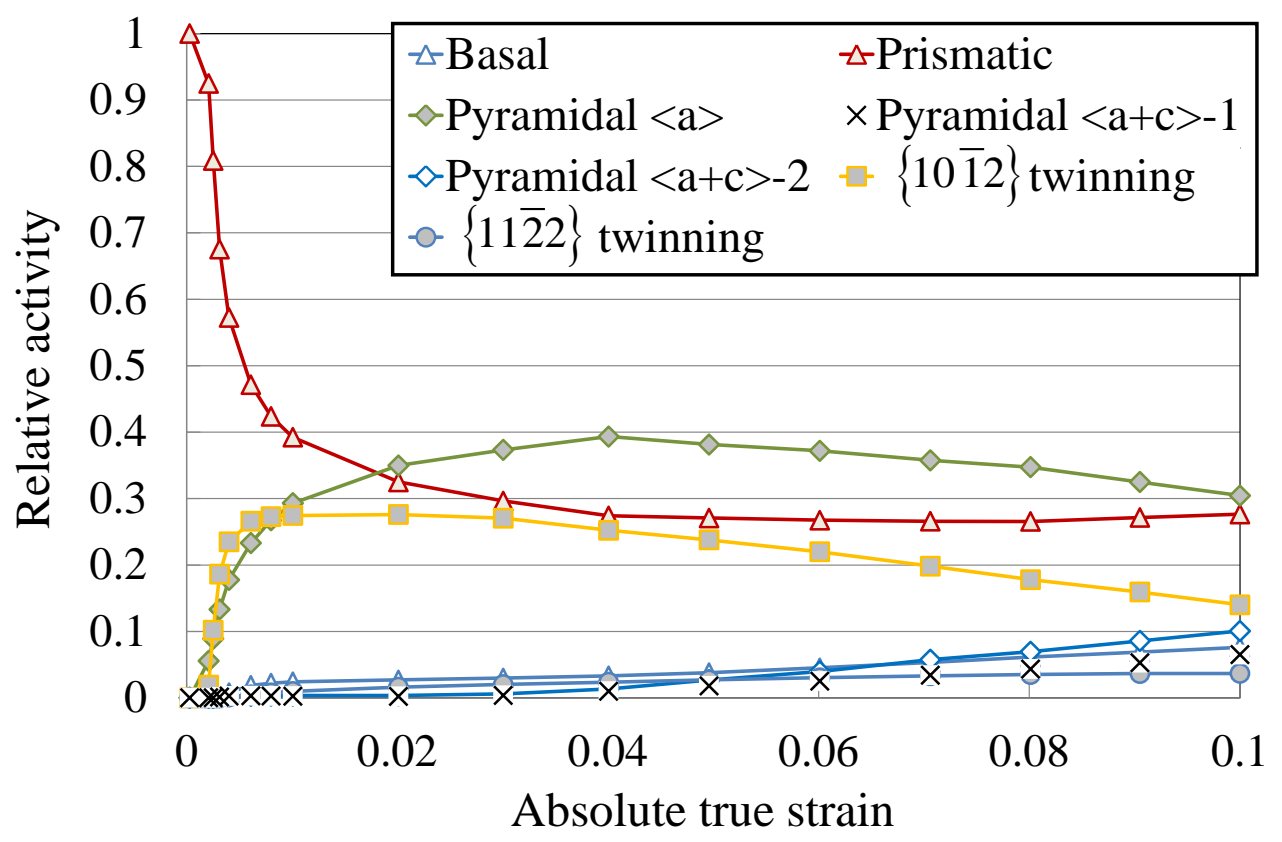

(b)

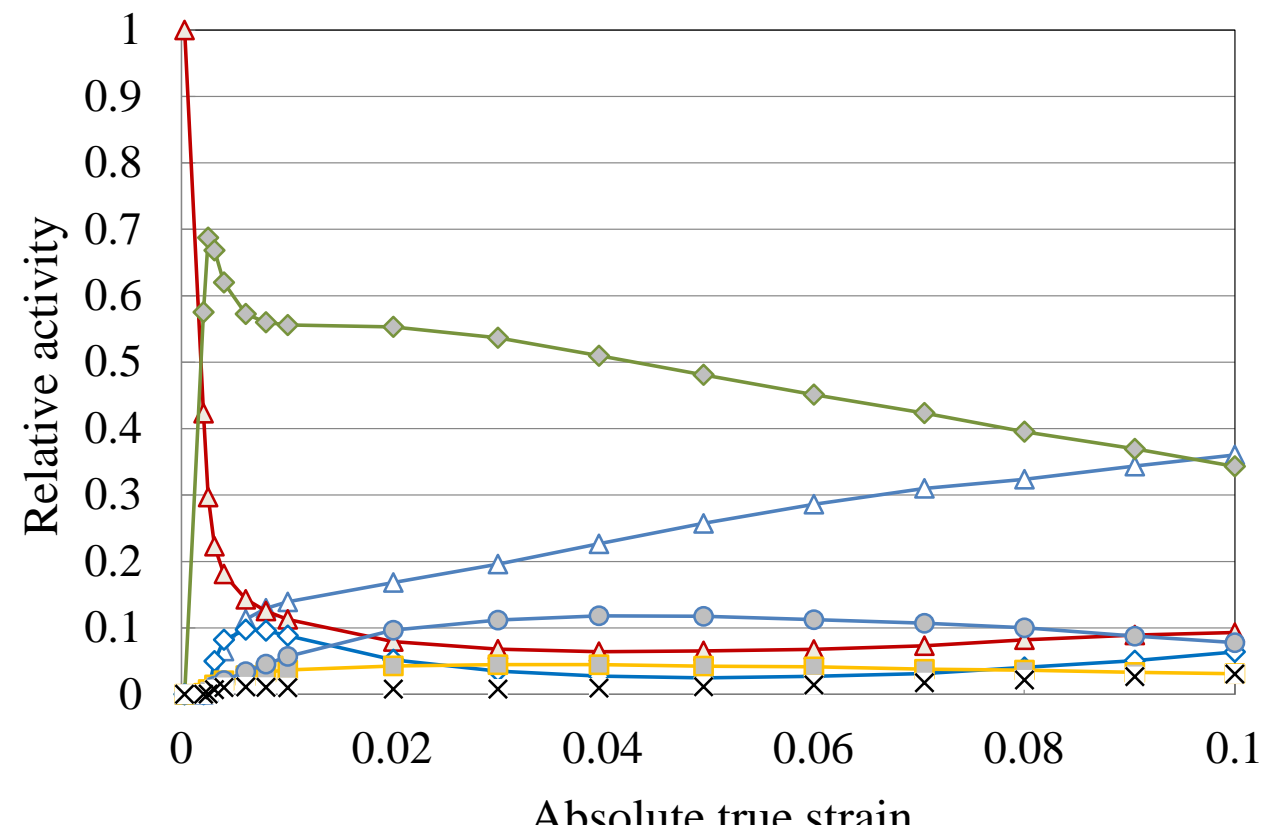

Fig. 26 Evolution of relative activities obtained under compression in (a) RD and (b) TD. 
(a)

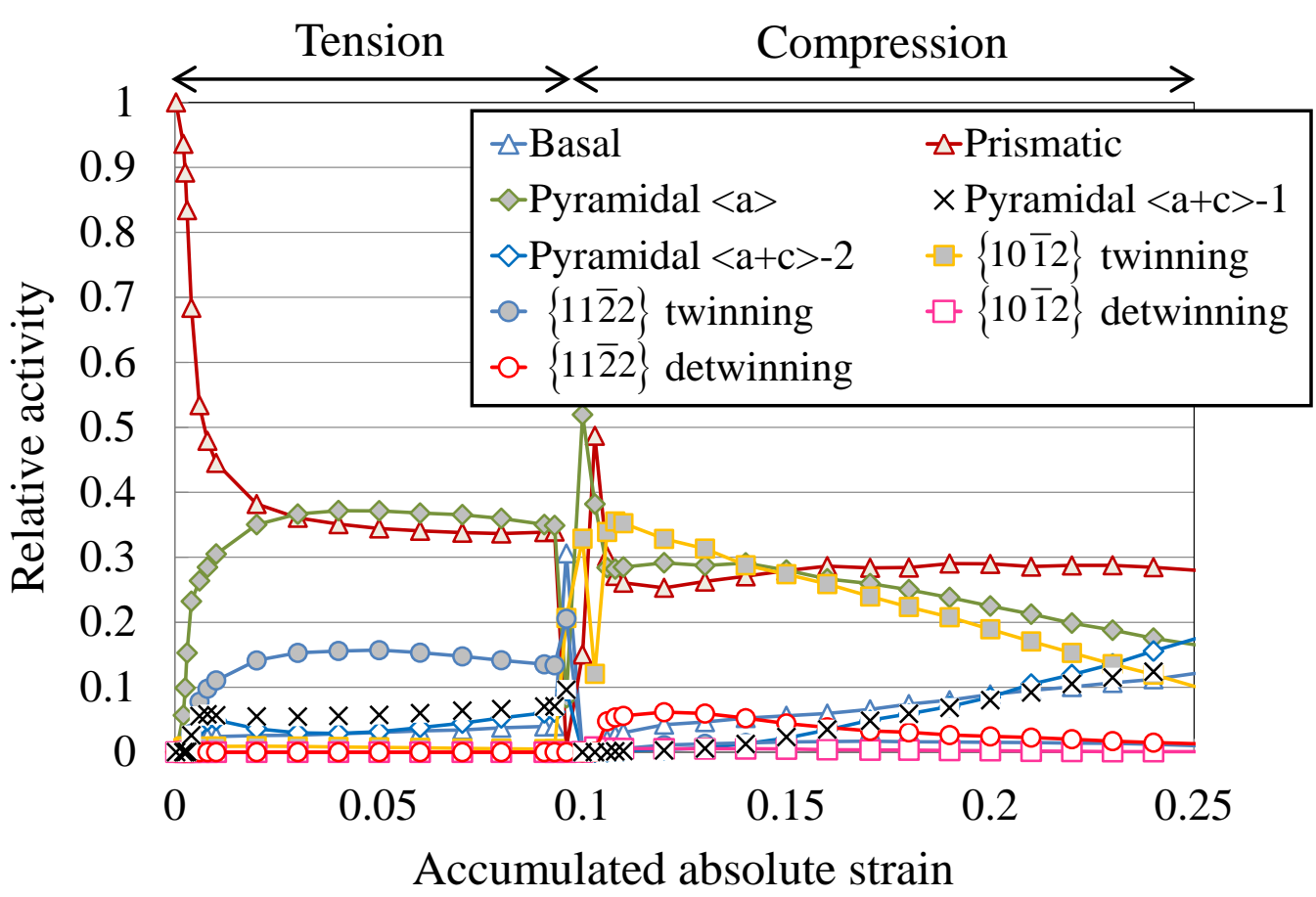

(b)

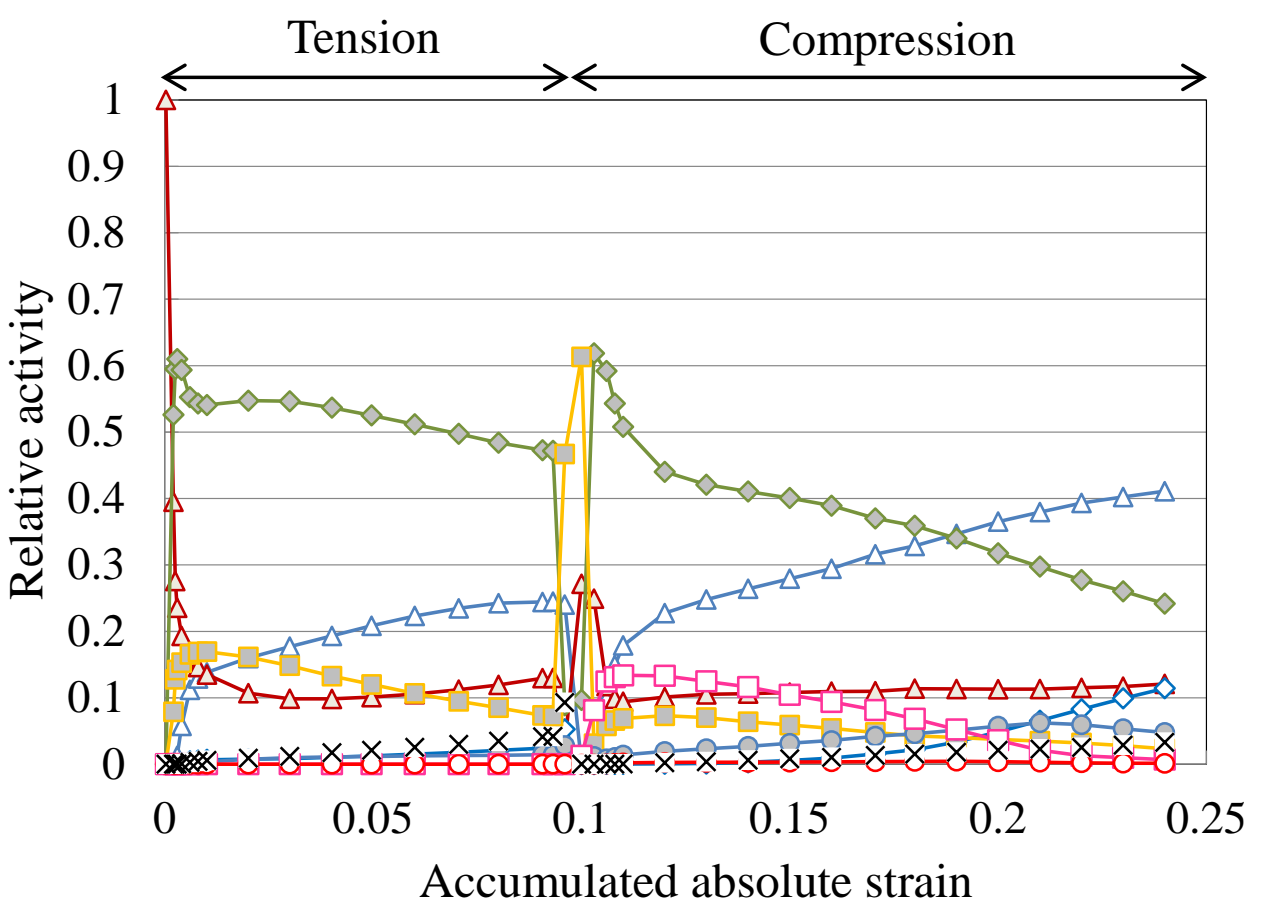

Fig. 27 Evolution of relative activities obtained under TC loading in the case of tensile pre-strain of 0.1 in (a) RD and (b) TD. 
(a)

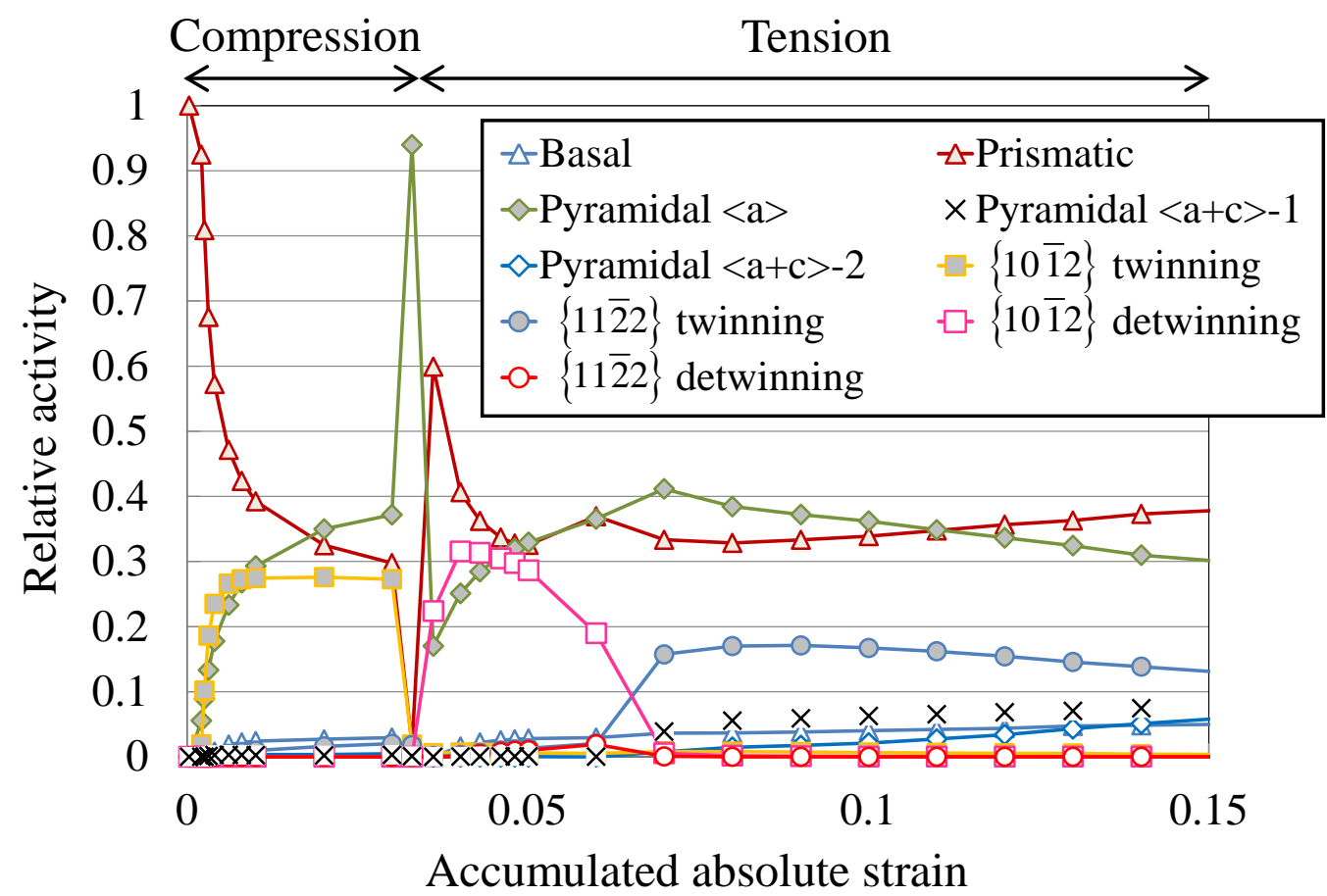

(b)

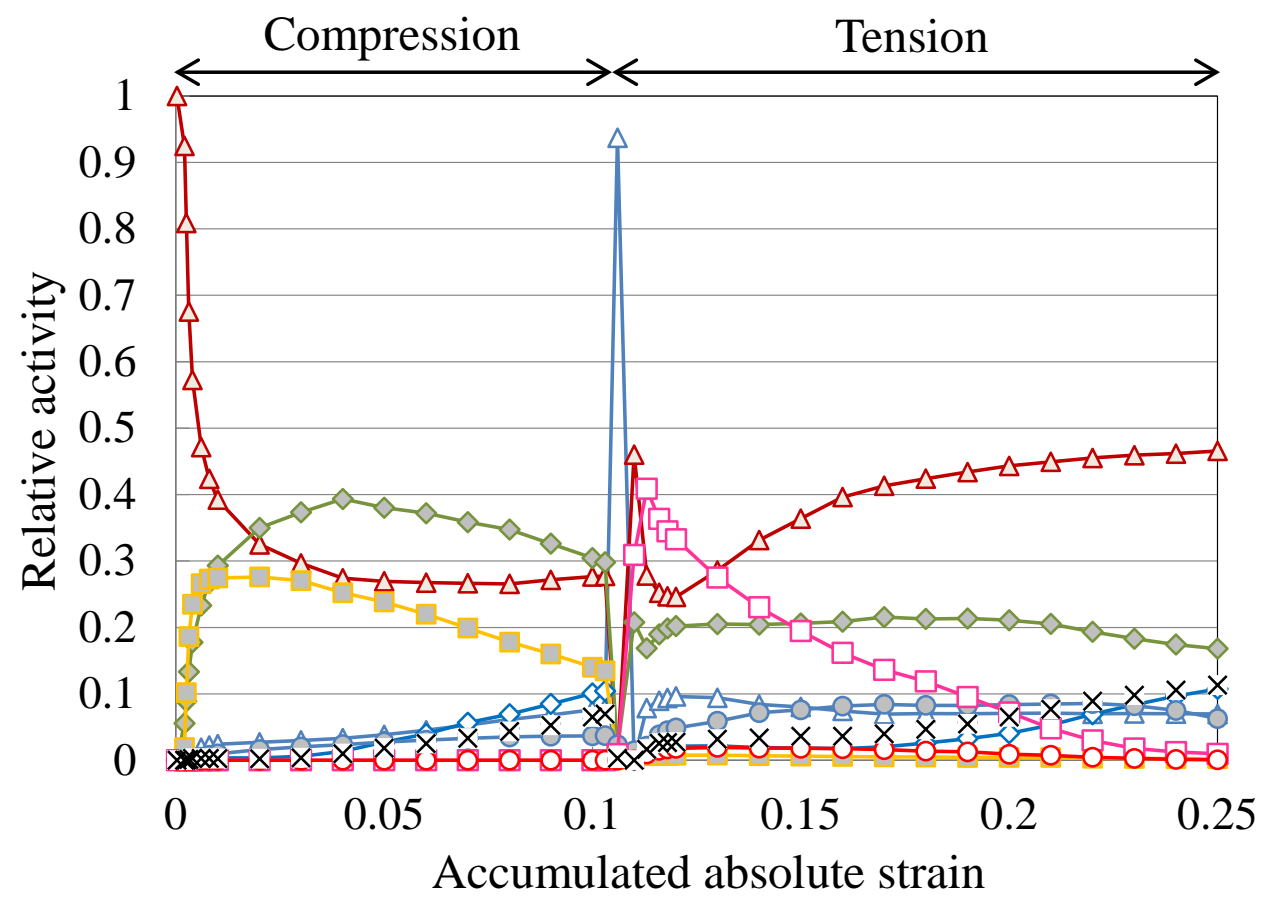

Fig. 28 Evolution of relative activities obtained under CT loading in RD in the case of compressive pre-strain of (a) 0.03 and (b) 0.1 . 
(a)

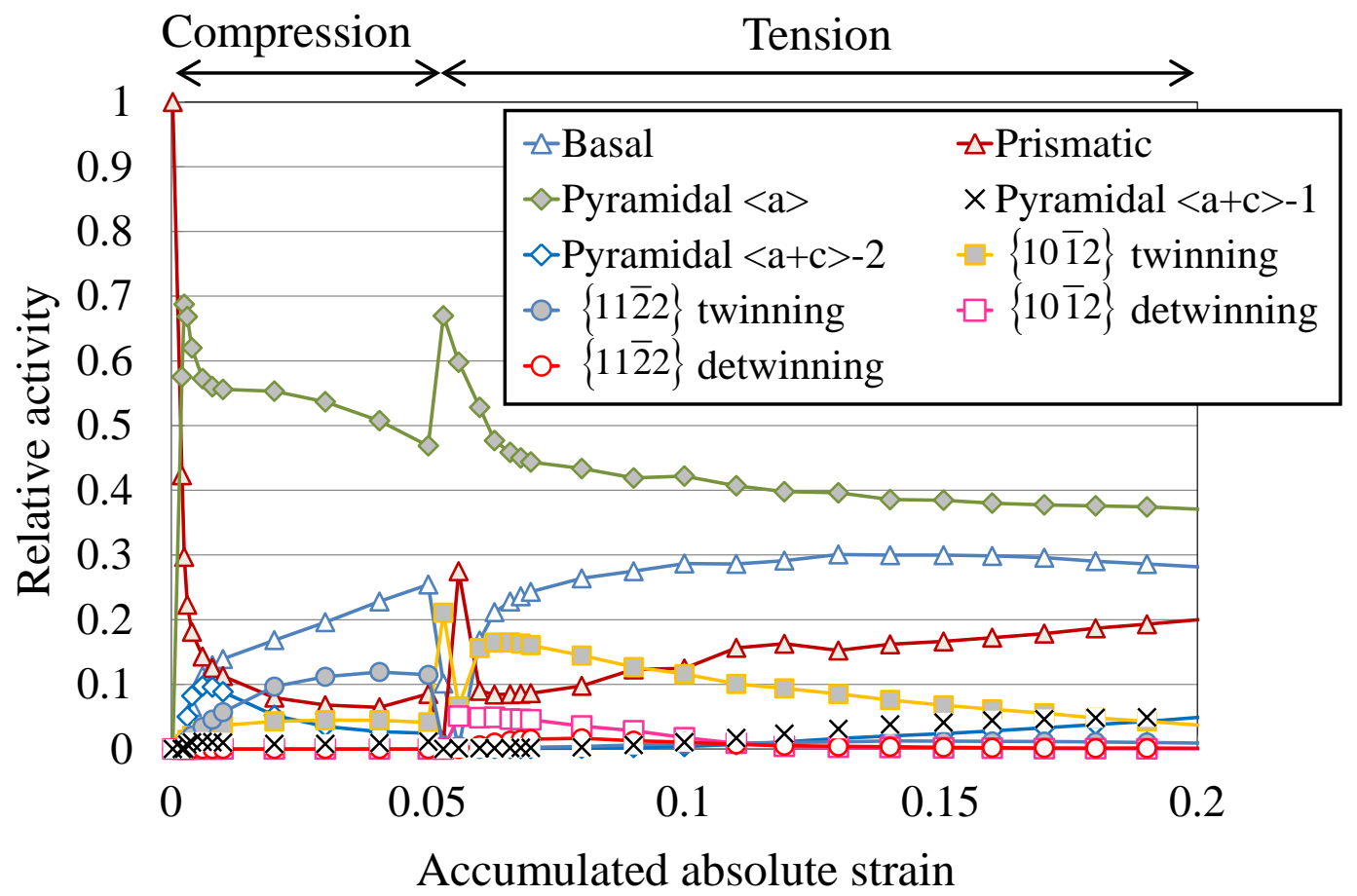

(b)

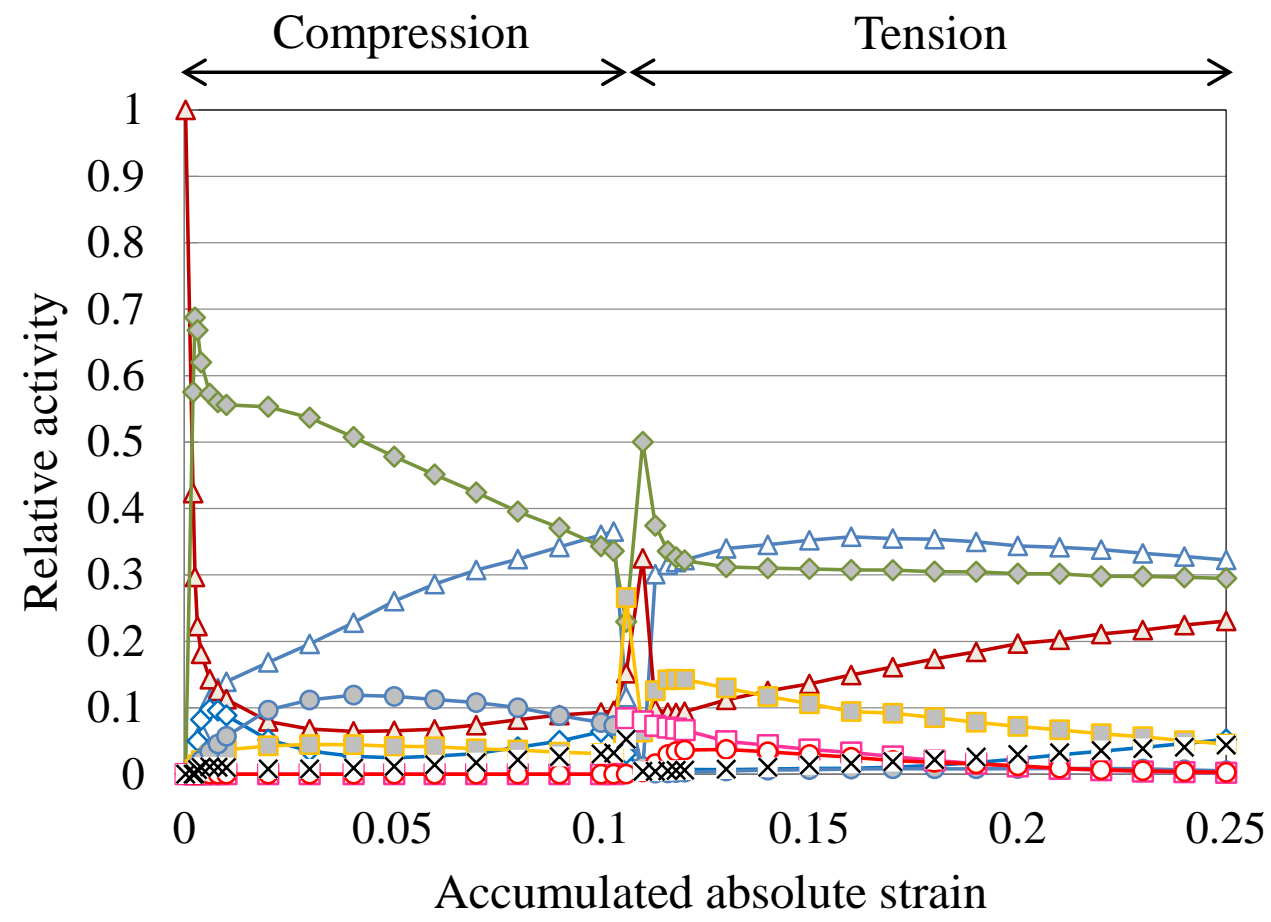

Fig. 29 Evolution of relative activities obtained under CT loading in TD in the case of compressive pre-strain of (a) 0.05 and (b) 0.1 . 